\title{
Performance limits in optical communications due to fiber nonlinearity
}

\author{
A.D.Ellis ${ }^{1 *}$, M.E.McCarthy ${ }^{1,2}$, M. A. Z. Al Khateeb ${ }^{1}$, \\ M.SOROKINA ${ }^{1}$, N.J.DORAN ${ }^{1}$.
}

${ }^{1}$ Aston Institute of Photonic Technologies, Aston University, Aston Triangle, James Watt Queensway, Birmingham, B4 7ET, United Kingdom.

${ }^{2}$ Now with Oclaro Technologies Ltd, Westfield Business Park, Paignton, TQ4 7AU, United Kingdom

*Corresponding author: andrew.ellis@aston.ac.uk

Received Month Day, Year; revised Month Day, Year; accepted Month Day, Year; published Month Day Year (Doc. ID xxxxx)

\begin{abstract}
In this paper, we review the historical evolution of predictions of the performance of optical communication systems. We will describe how such predictions were made from the outset of research in laser based optical communications and how they have evolved to their present form, accurately predicting the performance of coherently detected communication systems. (@) 2015 Optical Society of America
\end{abstract}

OCIS codes: (060.4510) Optical communications; (060.4370) Nonlinear optics, fibers; (060.1660) Coherent communications; (060.2330) Fiber optics communications.

http://dx.doi.org/10.1364/AOP.0.000000

\section{Introduction}

Current perceptions of fiber optic communication systems is that there is a practical, and impending, limit on the data throughput of a single mode fiber. This limit has been commonly called nonlinear Shannon limit [1]-[2] and such a fixed limit, when combined with continued exponential increase in demand for communication (at an almost constant compound annual growth rate of almost 40\% since 1999 [3]) results in predictions of an optical capacity crunch [4], a term which was first applied to communication networks at the start of the decade [5]. The relentless exponential growth in demand for data services, particularly video has, since 1975 [6], been largely fulfilled by using new technology to increase the capacity of a single optical fiber. Intentionally, business reality ensured that each successive technology generation would offer higher data rates, with reduced cost and energy consumption per bit. Energy efficiencies (per bit) typically improved at a rate of $20 \%$ per annum, continuing trends which have been enjoyed since Marconi's introduction of a wireless transatlantic service [7]. However, with demand increasing at $40 \%$, and efficiency gains lagging behind at $20 \%$, as with all exponential growth phenomena, something will eventually have to change. The timing of this change is, of course, is a debatable point, with simple graph plotting suggesting that unchecked growth in communications energy consumption could result in network's energy demands exceed global electricity production capability in the foreseeable future, whilst recent successful actions by major telecoms operators to constrain energy through decommissioning old equipment use could postpone the issue by up to a decade [8]. Unless there is a change in the rate of increase of demand, the inevitable change of business model into a new regime of finite resources will clearly be a challenge for carriers, service providers, and equipment manufacturers alike. The "post crunch" solution adopted by the industry will also have currently unpredictable consequences for consumers of communication systems, but these issues all fall beyond the scope of this article. 
Here, we focus on models which have rapidly become established and which may be used to predict the maximum performance of the ubiquitous single mode optical fibers used in major telecommunications networks. The anticipated performance limit is a fundamental consequence of the basic physics of any optical system, in particular the trade-off between noise, finding its origins in quantum mechanics, and nonlinearity usually described using electromagnetism. In many ways therefore, the performance limits of single mode optical fiber are fundamental consequences of modern physics. Optical amplifiers are close to the so called "quantum limit", and the susceptibility tensor of silica based fibers has a collection of characteristics which are hard to improve upon. However, even if new materials' science uncover a medium with even more favorable parameters we believe that the approach presented here will remain valid, and that the potential performance limits readily scaled from the material properties [9].

Predictions of the performance limits of optical communication systems are not new, and date back almost to the demonstration of the laser [10], and were even included in the first proposals for optical fiber [11]. Of course, in order to understand the performance limits of optical fiber communication systems, we must first understand our definition of those limits. Quantitative values of the acceptable performance limits have of course evolved with time, thanks in a large part in our ability to accept transmission errors due to improvements in forward error correction coding. However, the qualitative definitions have also evolved, and whilst a post error correction error rate lower than a set value, say less than 1 error over the entire length of the message can be readily universally established, they are hard to measure. Various proxy measurements have been proposed over time, and section 2 of this paper attempts to explain their relationships, potential confusions, and how to translate between them. As a broad overview, in section 3 and 4 we will discuss the performance limits of directly and coherently detected transmission systems limited by noise and/or the nonlinear Kerr effect, developing a concept of a fundamental performance limit. In section 5 we immediately describe how this apparent limit may be overcome, and a new limit is established. Before considering the potential benefits of moving towards this new limit in section 7, we briefly review nonlinear effects based on scattering phenomena in section 6 .

Section 3 commences with the linear performance limits for direct detection systems. In line with the earliest calculations [10] we include free space propagation as a potential source of loss, establishing fundamental performance limits considering the fundamental noise sources and simple practical limits on transmitted output power, illustrating the performance limits for both binary and non-binary digital communication systems. We then examine the impact of fiber dispersion and nonlinearity, considering the key impairments of self-phase modulation and parametric noise amplification, with the later involving an interaction between signal and noise and proving to be fundamental. The interplay between dispersion and nonlinearity gives rise to intricate optimization problems and fundamental performance limits which still appear to hold today, in the context of system design for short reach applications such as intra data center links. We next consider optical solitons, which offer the prospect of allowing dispersion and nonlinearity to balance out, but resulting in performance dominated by the interplay between signal and noise. Finally section 3 considers wavelength division multiplexed systems, where interactions between independent channels are added, and the concept of dispersion management is introduced. Dispersion management allows the conflicting requirements of minimizing parametric noise amplification, selfphase modulation and inter channel effects such as cross-phase modulation to be addressed simultaneously, but as we will see, performance limits remained, although they are difficult to calculate exactly.

Section 4 follows a similar structure, but in the case of coherent detection. It first considers the linear, noise limited performance limits of a communication system before considering nonlinearity. Whilst early work on coherent transmission systems followed the same path as direct detection wavelength multiplexed systems, there was little commercial interest until the performance of the simpler direct detection systems had been exhausted, including the implementation of super-channels to allow high information spectral densities. Thus the context facing the calculation of nonlinear transmission limits is somewhat changed, and we assume in this section that all of the known techniques to maximize the throughput of a linear communication system have been applied. Operation at a high information spectral density, with no or negligible guard bands between many independent channels gives rise to the concept of a nonlinear noise spectral density, and this is developed here in the frequency domain by integrating four wave mixing efficiencies over the signal bandwidth. Whilst this approach is perfectly general, provided that appropriate assumptions are included, in some circumstances the analytical solutions are lengthy, and alternative calculation methods may be preferred. Section 4 concludes with a survey of some of these methods.

In section 5, we speculate on the compensation of any nonlinear impairment which could, in principle at least be compensated. We consider three different methods, compensating the nonlinearity at the transmitter or receiver (or preferably both) in section 5.1, compensating the nonlinearity using optical signal processing distributed along the transmission link in section 5.2, and compensating the nonlinearity by transmitting mathematically related copies of the signal along ideally identical transmission links in section 5.3. We show in particular that once the deterministic nonlinearity is 
accounted for, the system is again limited by the interaction between signal and noise. Given this we also show that the highest performance gains are obtained when the compensation of the deterministic intersignal nonlinear effects is carried out bearing in mind the impact on the nonlinear interaction between signal and noise.

In section 6, we briefly review scattering nonlinearities, such as stimulated Raman and Brillouin scattering, concluding that whilst it is easy to design system which are limited by such effects (by eliminating dispersion, or transmitting strong carriers respectively) conventional systems without nonlinearity compensation are not constrained by these effects. Finally in section 7 we briefly speculate on the potential benefit of developing tools to compensate for the nonlinear effects limiting the performance of optical fiber systems, predicting a factor of two saving in the number of fibers required for a high capacity network. We hope that a consistent presentation of the various performance limits will lead to an understanding of the fundamental limits of each system design, and understanding of the changing (sometimes reversing) trends in design as we evolved our systems towards these limits, highlight what remains to be done and, most importantly, aid in the planning of post capacity crunch networks.

\section{Performance characterization.}

Assuming that all noise sources may be represented as independent random variables with a Gaussian distribution (additive white Gaussian noise), it is common for the performance of the systems to be characterized by a single parameter. For a hard-decision based system, where the probability of an error is given by integrating the tail of the Gaussian noise distributions extending beyond the decision threshold the statistical Q-function, or tail probability of the standard normal distribution is used, modified so that it takes into account errors crossing the decision threshold in both directions [12]. For a direct detection system, a factor $Q$ is defined as $Q=\left(\mu_{1}-\mu_{0}\right) /\left(\sigma_{1}-\sigma_{0}\right)$ where $\mu_{i}$ represents the amplitude of the $i^{\text {th }}$ level and $\sigma_{i}$ its standard deviation. For a binary system, this definition is well defined, and the one-to-one equivalence between $Q$ signal-to-noise ratio (snr) and bit error ratio (BER) is well understood. $Q$ factors were often calculated from eye diagrams recorded on digital sampling oscilloscopes or from bit error rate version decision threshold characteristics. Even today performance of binary systems is often quoted in terms of a $\mathrm{Q}$ factor, even if the BER was originally measured.

For non-binary systems, the relationship between a parameter derived from the statistical Q-function (rather than the parameter $Q$ used for direct detection systems), the snr and BER changes for each modulation format [13]. This can be seen by examining the hard-decision performance predictions for a rectangular constellation with $m$ constellation points

$$
B E R=\frac{2}{\log _{2}(m)}\left(1-\frac{1}{\sqrt{m}}\right) \operatorname{Erfc}\left(\sqrt{\frac{3 \cdot \operatorname{snr} \cdot \log _{2}(m)}{2 \cdot(m-1)}}\right)
$$

Where Erfc represents the complementary error function. There may well be implementation penalties which mean that the expected level of performance from a given signal to noise ratio is not often achieved, and the standard system characterization would be to plot BER as a function of detected snr or even more commonly optical signal-to-noise ratio (OSNR), and originally received signal power as a proxy for snr. However, in order to avoid the use of a double-log axis, the results are typically represented by a "Q-factor". To obtain this modified Q-factor from a BER (for any constellation), one solves the binary version of Equation 1:

$$
B E R=\frac{1}{2} \operatorname{Erfc}\left(\frac{Q}{\sqrt{2}}\right)
$$

It is perhaps both confusing and unfortunate that the same symbol has been used for this quasi logarithmic proxy for BER as for the well-defined binary Q-factor, however this is now standard practice. As we will see below, analytical predictions often deliver the snr, making direct comparison with experimental Q indirect. However, a simple translation between "Q" and snr is possible by solving Equations 1 (and equivalents for alternative modulation formats) and 0B, and an example is shown in Figure 1 below. An alternative approach, with a stronger parallel to the use of the binary Q-factor, is the use of the error-vector-magnitude (EVM) [14]. Direct detection Q factor computes the relationship between the minimum distance between points, and the sum of the standard deviations of the noise on these points. Conceptually, for error vector magnitude, this is replaced with the ratio of the minimum Euclidean distance to the noise standard deviation. If the constellation is uniform, and the noise is not pattern dependent, this is a simple definition. A strong correlation between BER and EVM has been reported for a wide range of optical system configurations [15], fully in line with theoretical predictions from more general communication theory. EVM may also be plotted in a logarithmic scale, and offers the useful advantage of allowing a smooth translation on a single graph from an equivalent EVM inferred from 
actual BER measurements, and EVM calculated from the statistical distribution of received constellation points.

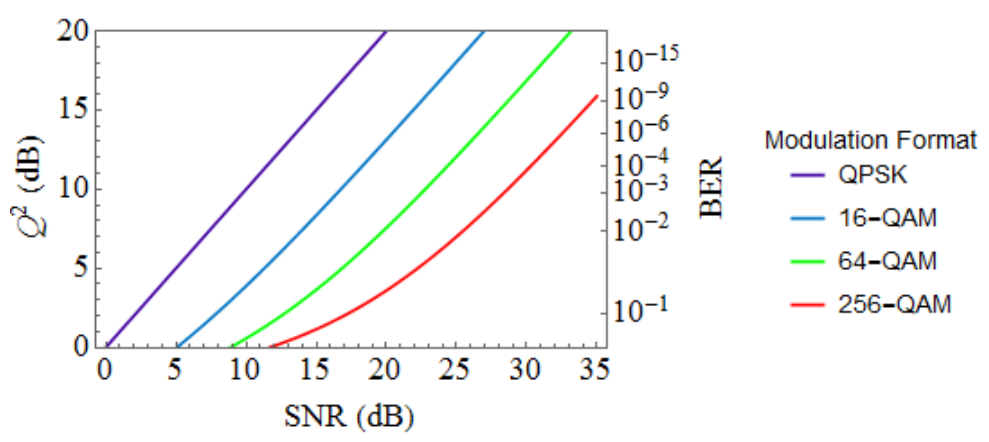

Figure 1: Theoretical relationship between experimentally reported Q-factor, BER and theoretically predicted signal-to-noise ratio for three commonly reported constellations.

In the case of soft decision decoding, utilized in many of today's FEC enabled systems, a performance metric fundamentally derived from hard decision is somewhat unsatisfactory. Despite this, much progress has been made where experimental results have been reported as a Q-factor whilst assuming that soft decision enabled FEC will successfully operate, and many high profile publications persist in this approach. The potential pitfalls of using a hard decision metric for a soft-decision system were pointed out recently, indicating that the practice can, in certain circumstances, give pessimistic results [16]. Mutual Information, and Generalized Mutual Information have been proposed as more accurate performance metrics to ascertain what system throughput would be possible if an appropriate error correcting code could be deployed. This approach undeniably overcomes some of the more obvious problems with $Q$, but unless fully flexible code adaptive hardware is envisioned introduces new problems of its own.

\section{Performance limits of direct detection communication systems}

\subsection{Linear performance of single channel optical communication systems.}

Shortly after the laser was first demonstrated many of the basic principles of electronic communication were translated to the realm of optical communication, and the theory of fiber optic waveguide propagation proposed and formalized $[11,17]$. The most immediate and significant advantage of laser based optical communications was the shot noise limited performance [10]. The further benefit of the ability to perform coherent detection was also quickly recognized [18]. The combination of shot noise and loss, either from scattering in an optical fiber or free space divergence readily allows the maximum transmission reach between regenerators to be estimated for a given performance for direct detection systems.

$$
Q^{2}=\left\{\begin{array}{rr}
\frac{\eta_{D} P_{T} A_{R}}{2 q B_{r} \Omega_{T} L^{2}} & \text { divergence } \\
\frac{\eta_{D} P_{T} e^{-\alpha L}}{2 q B_{r}} & \text { scatter }
\end{array}\right.
$$

where $P_{T}$ represents the transmitted power, $\eta_{D}$ and and $B_{r}$ the receiver quantum efficiency and bandwidth respectively. $A_{R}$ represents the effective area of the receiver aperture, $q$ the charge on the electron, $\Omega_{T}$ the source divergence, $L$ the transmission distance and $\alpha$ the fiber loss. Such equations represent perhaps the earliest estimations of the ultimate performance of an optical communication system. This approach led to predictions of $200 \mathrm{~km}$ long free space communication channels with bandwidths of $100 \mathrm{GHz}$ using only $1 \mathrm{~mW}$ transmitter launch power (at a signal to noise ratio of 16dB) [10]. A guided wave system could offer similar performance over a comfortably competitive $50 \mathrm{~km}$, provided the scatter loss coefficient was less than $20 \mathrm{~dB} / \mathrm{km}$ and the signal launch power below the likely damage threshold of the waveguide [11], a target readily achieved a few years later [19, 20]. Of course, in practical systems the signal to noise ratio was degraded by receiver thermal noise and various beat noise terms originating from amplified spontaneous emission from optical amplifiers. Thus whilst [11] provides a 
performance estimate, more accurate performance predictions were made possible by analyzing these additional noise sources, and have been carried out for coherent detection, direct detection, optical time division multiplexed, and optically amplified transmission systems [18, 21-23]. Loss was always the dominant limiting factor and drove the transmission wavelength from the convenient to a local fiber loss minimal in the region of $1300 \mathrm{~nm}$ (the second telecommunications window, see [24]) and eventually to the lowest loss window in the region of $1550 \mathrm{~nm}$, where the impact of chromatic dispersion became apparent as bit rates rose. The dominance of loss allowed the impact of the majority of impairments to be modelled as a signal-to-noise ratio (or equivalently eye opening) penalty. Such impairments included mode partition noise [25], transmitter extinction ratio, chromatic dispersion and polarization mode dispersion. Dispersive effects are fundamentally determined by the symbol rate of the system, but these early systems were often dominated by additional effects induced by chirp. As symbol rates exponential grew with time, to avoid increasingly troublesome penalties from chromatic dispersion whilst retaining the benefits of low loss transmission in the $1550 \mathrm{~nm}$ window, dispersion shifted fiber was introduced. This was particularly important for optically amplified systems, where the improved signal-to-noise ratio allowed both higher symbol rates and increased spacing between regenerators increasing the impact and quantity of chromatic dispersion respectively. For a simple direct detection system, it is straightforward to calculate the signal to noise ratio of the detected signal taking into account all of these terms. Normalizing each noise term to the signal photocurrent $\left(I_{s}\right)$ we have noise contributions from shot noise (both from the signal $\left(I_{s s h}\right)$ and the amplified spontaneous emission $\left(I_{\text {ash }}\right)$, beat noise between signal and amplified spontaneous emission $\left(I_{s a}\right)$, beat noise between different components of the amplified spontaneous emission (Iaa), and thermal noise (Ith) [26];

$$
\begin{gathered}
I_{s} \propto P_{s}^{2} \\
I_{s s h} \propto P_{s} P_{e} B_{r} \\
I_{a s h} \propto P_{a} P_{e} B_{r} \\
I_{s a} \propto P_{s} P_{a} \frac{B_{r}}{B_{S}} \\
I_{a a} \propto P_{a}^{2}\left(\frac{B_{r}}{B_{S}}-2 \frac{B_{r}^{2}}{B_{S}^{2}}\right) \\
I_{t h} \propto \frac{k_{B} T}{q^{2} R_{L}} P_{e}^{2} B_{r}
\end{gathered}
$$

where

$$
P_{a}=N_{a}(g-1) n_{s p} h v B_{S}
$$

and

$$
\boldsymbol{P}_{e}=\frac{\boldsymbol{h} v}{\boldsymbol{\eta}_{D}}
$$

Where $P_{S}$ represents the mean signal power incident on the photodetector, $P_{a}$ the total amplified spontaneous emission power incident on the photodetector (assuming a chain of $N_{a}$ identical amplifiers of gain $g$, filtered bandwidth $B_{S}$, and spontaneous emission parameter $n_{s p}$ ) $P_{e}$ the equivalent incident power of a single photoelectron. For the thermal noise contribution, $k_{B}$ represents Boltzmann's constant, $T$ the equivalent temperature and $R_{L}$ the equivalent trans-impedance amplifier load resistance. Taking into account these terms, and the impact of finite extinction ratio $\left(\sigma_{D}\right)$ from transmitter imperfections of dispersion, the performance of a binary on-off keyed system with direct detection may be predicted from [27]:

$$
Q_{D D-2}=\frac{1-\sigma_{D}}{1+\sigma_{D}} \frac{\sqrt{I_{s}}}{\sqrt{\frac{I_{s s h}+I_{s a}}{1+\sigma_{D}}+I_{a s h}+I_{a a}+I_{t h}}+\sqrt{\sigma_{D} \frac{I_{s s h}+I_{s a}}{1+\sigma_{D}}+I_{a s h}+I_{a a}+I_{t h}}}
$$

In the majority of circumstances, a simplified form is considered. For example, for a low cost system only thermal and signal shot noise terms would be considered, whilst for a long haul optically amplified system these terms, plus spontaneous-spontaneous beat noise terms are often neglected. In these two circumstances, equation 5 becomes

$$
Q_{D D-2}=\frac{1-\sigma_{D}}{\sqrt{1+\sigma_{D}}} \frac{\sqrt{I_{s}}}{\sqrt{I_{s s h}+\left(1+\sigma_{D}\right) I_{t h}}+\sqrt{\sigma_{D} I_{s s h}+\left(1+\sigma_{D}\right) I_{t h}}}
$$

and

$$
Q_{D D-2}=\frac{1-\sigma_{D}}{\sqrt{1+\sigma_{D}}\left(1+\sqrt{\sigma_{D}}\right)} \frac{\sqrt{I_{s}}}{\sqrt{I_{s a}}}
$$


respectively.

Equation 5 is usually derived for a two level (on-off keyed) system, for M-PAM systems a similar approach may be taken by optimizing the amplitude levels such that the contributions to the biterror rate for errors between each pair of adjacent levels are equal. For signals dominated by signal independent noise such as receiver thermal noise this results in equally spaced levels in power, whilst for systems dominated by signal dependent noise such as optically amplified systems this approach results in equally spaced levels in field amplitude (quadratically spaced in power). The system performance is then

$$
Q_{D D-M(i)}=\frac{Q_{D D-2}}{M-1}
$$

For systems dominated by signal dependent noise [28] and following the same approach

$$
Q_{D D-M(d)}=3 \frac{Q_{D D-2}}{(2 M-1)(M-1)}
$$

Taking into account the transition probabilities and Grey coding the required received signal power should be adjusted by between $3.3 \mathrm{~dB}$ (signal independent noise) and $6.9 \mathrm{~dB}$ (signal dependent noise) to obtain the same bit-error-rate. In practical terms this implies that an acceptable on-off keyed system could be upgraded to 4-PAM by the addition of forward error correction coding, whilst upgrades beyond this (for example to 8-PAM) would also require an increase in the signal to noise ratio.

\subsection{Nonlinear performance of single channel optical communication systems.}

Having established the baseline signal-to-noise ratio performance of an optical communication system, it is necessary to also consider pulse distortion [29], which would give rise to inter-symbol interference. Propagation of communication signals with symbol rates very much less than the carrier frequency (satisfying the slowly varying envelope approximation) are well modelled by the nonlinear Schrödinger equation [30];

$$
\frac{\partial u}{\partial z}=-\frac{\alpha}{2} u+j \gamma|u|^{2} u-\beta^{\prime} \frac{\partial u}{\partial t}-\frac{j}{2} \beta^{\prime \prime} \frac{\partial^{2} u}{\partial t^{2}}+\frac{1}{6} \beta^{\prime \prime \prime} \frac{\partial^{3} u}{\partial t^{3}}+\cdots
$$

Where $u$ is the optical field envelop, $\alpha$ is the loss coefficient of fiber, $\gamma$ is the nonlinear factor of the fiber (typically in the region of 1 to $1.4 / \mathrm{W} / \mathrm{km})$, and ( $\beta^{\prime}, \beta^{\prime \prime}$, and $\left.\beta^{\prime \prime \prime}\right)$ are the first, second and third order dispersion coefficients of the fiber respectively. $\mathrm{z}$ and $\mathrm{t}$ take their conventional space and time definitions respectively. Pulse evolution is typically dominated by the first and third terms on the right hand side of equation 10 (loss and group delay), although it is conventional to adopt a moving reference frame to minimize the impact of $\beta$ '. The second term represents nonlinearity, and becomes significant when the product of signal power (proportional to $|u|^{2}$ ) time transmission distance approaches $1 \mathrm{~W} . \mathrm{km}$. The dispersive terms ( $\beta^{\prime \prime}$, and $\beta^{\prime \prime}$ ') depend on the fiber type, but for standard single mode fiber, a value of $\beta^{\prime \prime}$ of around $-20 \mathrm{ps}^{2} / \mathrm{km}$ implies that, for a system with a symbol rate in the region of $10 \mathrm{Gbaud}$, the term becomes significant for system lengths exceeding $40 \mathrm{~km}$. Clearly, given the wide variety of potential system configurations, it is difficult in general to simplify equation 10, although for certain specific cases simplification is possible. For a non-return to zero system is difficult to exactly predict dispersion penalties analytically, since they are critically dependent on the optical and electrical filter bandwidths and the transmitted pulse shape. Dispersion penalties may be calculated from the broadened pulse width, and owing to the complexity of the pulse evolution for a non-return-to-zero signal this was sometimes performed empirically [31]. However, modelling a non-return to zero system as a sequence of superimposed Gaussian pulses allows dispersion penalties to be estimated by calculating the pulse power at the adjacent decision point in the same way that penalties in optical time division multiplexed signals may be calculated [32]. Treating the dispersed power level as a degraded extinction ratio for the adjacent symbol enables the length dependence of the penalty to be quantified using Equation 5, which takes into account both loss of pulse energy to adjacent time slots and inter symbol interference. Calculating nonlinear broadening first (without considering dispersion) and then examining the temporal evolution of the pulse [30] gives a normalised pulse power at the centre of the adjacent time slot, $\sigma$, given by the solution to

$$
16 \frac{\ln (2)^{2}}{\log _{2}\left(\sigma_{D}\right)}=1+\sqrt{2} \phi_{L} \phi_{N L}+\phi_{L}^{2}\left(1+\frac{4}{3 \sqrt{3}} \phi_{N L}^{2}\right)
$$

where

$$
\begin{array}{r}
\phi_{L}=16 B_{T x}^{2} \beta^{\prime \prime} N_{a} L \ln (2)^{3} \\
\phi_{N L}=\gamma P_{S} N_{a} L_{e f f}
\end{array}
$$


and where $B_{T_{x}}$ represents the transmitted signal bandwidth, $L$ is the span length, $L_{e f f}$ is the fiber effective length $(1-\exp (-\alpha L)) / \alpha . \phi_{L}$ and $\phi_{N L}$ represent scaling factors for phase shifts induced by linear and nonlinear effects respectively. The approach is only valid for low levels of nonlinearity $\left(\phi_{N L}<1\right)$, but is applicable to low cost short reach systems and long haul systems with a low channel count and no dispersion compensation. The broad implications of equations 5 and 11 are shown by the solid curves in Figures 2 and 3 (plotted for normal and anomalous dispersion respectively) which illustrate three characteristic features. For low launch powers $\left(\phi_{N L} \ll 1\right)$, and sufficiently low dispersion $\left(\phi_{L} \ll 1\right)$, the signal to noise ratio simply increases linearly with signal launch power, as expected from equation 5 alone. For higher values of dispersion, a power penalty is introduced, which rapidly degrades the performance by approximately $10 \mathrm{~dB}$ for every order of magnitude increase in $\phi_{L}$ above 1 . Finally, at high power levels, additional phase distortion due to fiber nonlinearity (self-phase modulation) greatly enhances the impact of dispersion with the netresult of a quadratic decrease in performance with launched power. Today, such nonlinear threshold curves are a familiar feature of experimental reports of long haul transmission [3338 ], although they may be plotted in terms of bit error rate rather than signal to noise ratio or Q factor [3941].

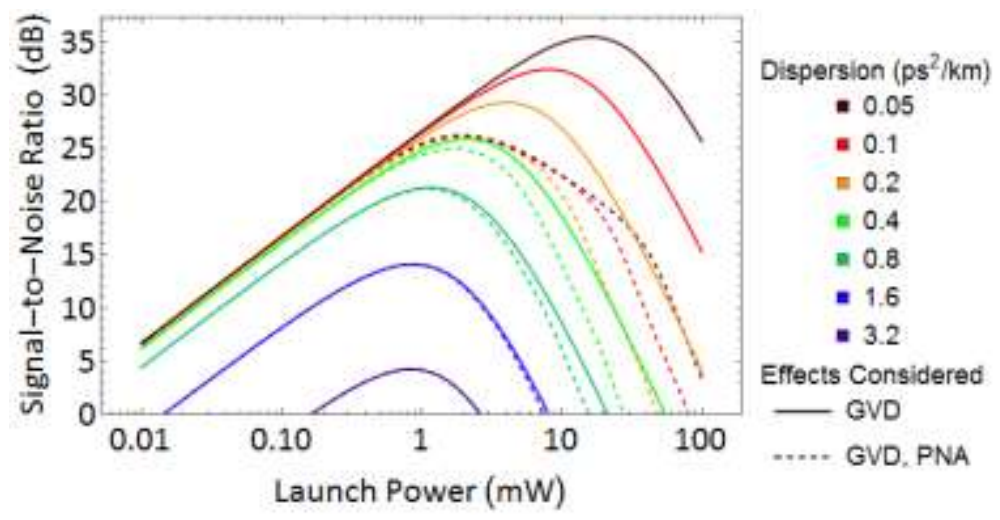

Figure 2: Predicted signal to noise ratio for a 10 Gbaud amplitude shift keyed system with direct detection over fifty $65 \mathrm{~km}$ spans of fiber assuming nonlinear coefficient of $1.4 / \mathrm{W} / \mathrm{km}$, loss of $0.2 \mathrm{~dB} / \mathrm{km}$, noise figure of $4.8 \mathrm{~dB}$ and normal chromatic dispersion with magnitudes of $3.2,1.6,0.8,0.4,0.2,0.1$ and $0.05 \mathrm{ps}^{2} / \mathrm{km}$ (purple to mauve respectively) based on self-phase modulation and dispersion (solid lines) and for self-phase modulation, dispersion and parametrically amplified noise (dotted lines).

The key difference between figures 2 and 3 is the interaction between dispersion and nonlinearity induced phase shifts. For normal dispersion (figure 2) the chirp acquired from these two effects add and only increases the rate of pulse broadening. For anomalous dispersion (figure 3), they have opposite signs and a small amount of pulse compression is possible, leading to reduced overall pulse broadening and higher signal-to-noise ratio. However, these effects are most clearly felt when the total accumulated dispersion is large (compared to the pulse width). The solid curves in figures 2 or 3 strongly suggest that arbitrarily high capacity could be achieved by simultaneously minimizing chromatic dispersion and increasing the signal launch power to a sufficiently high level. 


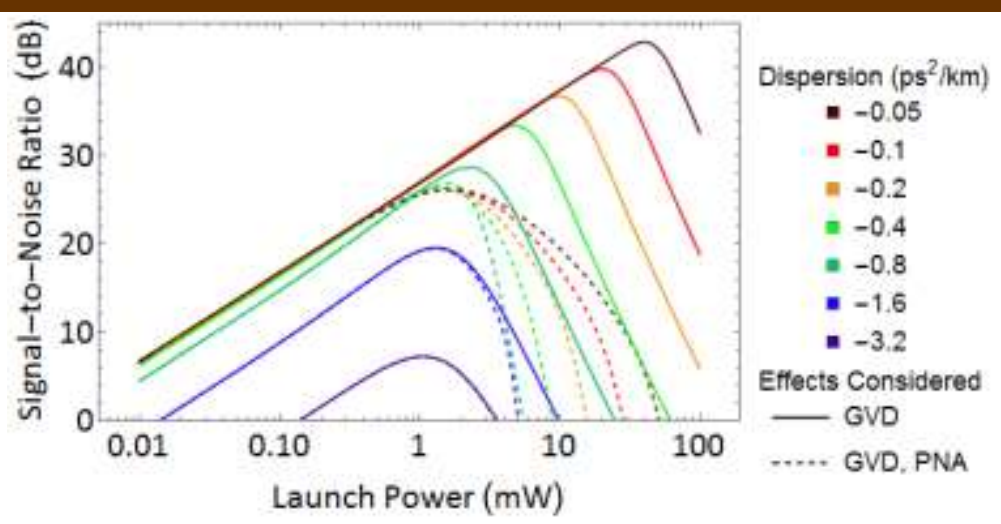

Figure 3: Predicted signal to noise ratio for a 10 Gbaud amplitude shift keyed system with direct detection over fifty $65 \mathrm{~km}$ spans of fiber assuming nonlinear coefficient of $1.4 / \mathrm{W} / \mathrm{km}$, loss of $0.2 \mathrm{~dB} / \mathrm{km}$, noise figure of $4.8 \mathrm{~dB}$ and anomalous chromatic dispersion with magnitudes of $3.2,1.6,0.8,0.4,0.2,0.1$ and $0.05 \mathrm{ps}^{2} / \mathrm{km}$ (purple to mauve respectively) based on self-phase modulation and dispersion (solid lines) and for self-phase modulation, dispersion and parametrically amplified noise (dotted lines.

Unfortunately, even before the practical considerations of fiber power handling and the necessary fiber fabrication precision to control dispersion are taken into account, additional nonlinear effects come into play to restrict the capacity at low dispersion, in particular the parametric interaction between signal and noise $[42,43]$ which was observed in the earliest experiments at $2.5 \mathrm{Gbit} / \mathrm{s}$, for straight line [39] systems, recirculating loops [44], and in numerical simulations [45]. The observed effects were attributed to the parametric amplification of the amplified spontaneous emission by the signal, which was sometimes referred to as modulation instability. The effect is critically dependent on the chromatic dispersion in the fiber section where the majority of the nonlinearity occurs, typically one effective length after each optical amplifier, rather than the average dispersion of the link. The process of parametric gain in a single mode fiber is well understood [30] including the effect of dispersion in enhancing the nonlinear effects through a process known as phase matching. Considering a small signal perturbative analysis, it is straightforward to show that the noise enhancement factor $F_{M I}$, which multiplies the amplified spontaneous emission noise $P_{a}$ in equations 4 , is

$$
F_{M I}=1+\frac{\phi_{N L}^{2}}{N_{a}^{2} \kappa_{m}^{2}}\left(\left(1-\frac{1}{2 N_{a}}\right)-\frac{\operatorname{Sin}\left(\left(2 N_{a}-1\right) \kappa_{m}\right)}{2 N_{a} \operatorname{Sin}\left(\kappa_{m}\right)}\right)
$$

Where the dispersive scaling parameter $\kappa_{\mathrm{m}}$ is given by

$$
\kappa_{m}=2 \sqrt{\beta^{\prime \prime} L} B_{T x} \pi \sqrt{\phi_{N L}+\beta^{\prime \prime} L B_{T x}^{2} \pi^{2}}
$$

In the limit of small dispersion (such that $\kappa_{m} \ll 1$ ) the noise enhancement grows quadratically with the number of cascaded amplifiers, severely limiting the performance of low dispersion systems. The combined impact of both self-phase modulation and parametric noise amplification are shown by the dashed lines in figures 2 and 3. For self -phase modulation limited systems (solid lines) it can be seen, from the higher peak signal-to-noise ratio in figure 3 when compared to that of figure 2 , that anomalous dispersion is preferred due to the slight pulse compression effect which is analogous to soliton propagation (see section 3.3). Conversely, due to nonlinear phase matching, systems limited by parametric noise amplification (dashed curves) show a preference for small, but finite, normal dispersion. Whilst only strictly only valid if $\phi_{N L} \ll \pi$ and critically sensitive to the exact system configuration, equations 5 and 11 allow the maximum capacity of a given system configuration to be estimated. Some examples are shown in figure 4 below where we consider the maximum achievable bit rate (for a target $\mathrm{Q}^{2}=15.5 \mathrm{~dB}$, corresponding to the typical bit error ratio target of $10^{-9}$ of early papers in this field) for low cost applications including client side interfaces, data centers and access networks for standard and dispersion shifted fibers (red), for single channel unrepeated systems using an optical pre-amplifier (blue), and for single channel systems with in-line amplifiers (purple). For the systems employing optical amplifiers (in-line and pre-amplifiers), we optimized both the signal launch power and the dispersion to maximize the peak signal to noise ratio for each point. For the in-line system, we fixed the amplifier 
spacing to 65km. Experimental results for binary systems (solid symbols) and more complex modulation formats (open symbols) all fall within their respective performance limits, even those employing forward error correction codes with target BERs in the region of $10^{-3}$. Consequently, it can be seen that, despite the wide variety of modulation formats used in practice, the guidelines derived from Equation 5 appear to be valid for contemporary transmission systems, may be used to identify the dominant impairment for any given bit rate and distance, and guide the choice of fiber and receiver characteristics. The theoretical modelling suggests that we may anticipate terabit class interfaces for transmission distances up to $10 \mathrm{~km}$ using either the $1310 \mathrm{~nm}$ transmission window (to minimize dispersion) or optical amplification and digital signal processing.

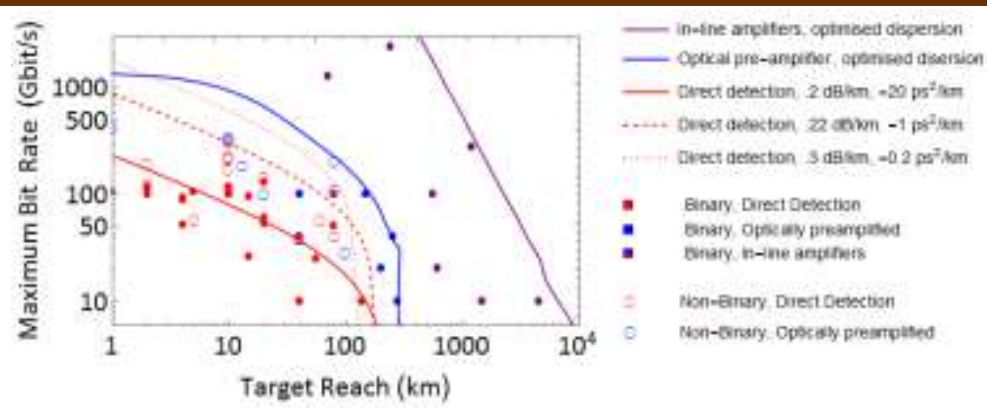

Figure 4: Maximum bit rate for single wavelength systems with direct detection as a function of transmission length over single mode fiber. Analytical predictions (lines) assume binary onoff keyed modulation for unrepeatered systems with single mode fiber (solid red, $-20 \mathrm{ps}^{2} / \mathrm{km}$, $0.2 \mathrm{~dB} / \mathrm{km}$ ) dispersion shifted fiber (dashed red, - $1 \mathrm{ps}^{2} / \mathrm{km}, 0.22 \mathrm{~dB} / \mathrm{km}$ ) at $1550 \mathrm{~nm}$, single mode fiber at $1310 \mathrm{~nm}$ (dotted red, $-0.2 \mathrm{ps}^{2} / \mathrm{km}, 0.3 \mathrm{~dB} / \mathrm{km}$ ) with direct detection receivers, for an unrepeated single mode fiber system (1550nm, $-20 \mathrm{ps}^{2} / \mathrm{km}, 0.2 \mathrm{~dB} / \mathrm{km}$ ) with an optical preamplifier and the optimum dispersion (blue), and for a system with in-line amplifiers spaced every $65 \mathrm{~km}$ with optimized dispersion (subject to a minimum of $0.2 \mathrm{ps}^{2} / \mathrm{km}$, purple). For unrepeated systems assume a fixed transmitter launch power of $10 \mathrm{~mW}$, whilst launch power is optimized for each point for in-line amplified systems. Dots represent reported per wavelength experimental results for low cost short reach systems (red), single channel systems with optical pre-amplifiers (blue) and in-line amplifiers (purple). See [46-50] for selection of references for binary amplitude shift keyed systems (solid symbols) and [51-71] for more complex formats (open symbols) operating with FEC.

Currently, research incorporating direct detection not only includes single mode fibers (as shown in figure 4), but also multimode fibers in order to minimize costs and simplify deployment strategies. Whilst new fiber types such as OM4 and OM5 significantly reduce modal dispersion, electronic equalization is often employed for these systems, increasing cost.

For a direct detection system with uniform dispersion, given that figure 2 suggests that there is little margin for $10 \mathrm{Gbit} / \mathrm{s}$ propagation over $1,625 \mathrm{~km}$ it is apparent that such systems are unlikely to find application in trans-continental and submarine transmission systems. In order to break the dispersion trade off resulting from these competing nonlinear effects, the concept of dispersion management was introduced, where large sections of the system comprised slightly anomalous dispersion fiber, minimizing the parametric noise amplification effect and shorter section of positive dispersion fiber (for example standard single mode fiber) were used to maintain a low overall path averaged dispersion to minimize self-phase modulation pulse broadening [72]. Alternative maps were soon also proposed [eg 73] with similar levels of performance. The concept of dispersion management had been proposed and experimentally observed [74, 75] for soliton systems, where the benefits of nonlinear transmission combined with anomalous dispersion were fully exploited to eliminate pulse distortion. Whilst concepts such as map strength, first introduced for solitons [76] were eventually applied to non-soliton systems for many years system designers resorted to complete numerical simulations rather than direct calculation of performance limits, and the earliest single channel optically amplified transmission systems employed dispersion management to avoid parametric noise amplification [77]. Whilst coherent transmission systems have reduced the requirement for dispersion management in today's systems, research on dispersion management persists to deal with the limits of finite signal processing memory [78], legacy fiber [79], reduce cost in access systems [80] and enable the use of low cost transponders [81]. 


\subsection{Soliton transmission systems}

In this section, we consider the transmission performance of a specific class of optical transmission system known as a soliton transmission system. As we saw in the section above, it is possible to calculate the performance limits of a system by studying the evolution of optical pulses. In particular Equation 11 may be used to design a transmission system which minimizes the pulse distortion arising from the combination of nonlinearity and chromatic dispersion at a particular transmission distance and launch power. The net effect is close to a balance between dispersion and nonlinearity which maximizes the performance. However, it can be shown analytically for a lossless fiber that for certain pulse shapes the balance between dispersion and nonlinearity is exact and occurs continuously along the fiber length. Equation 10 may be solved directly in order to find these solutions, which are known as solitons, where the pulse intensity remains invariant with transmission distance, neatly balancing out the effects of selfphase modulation and chromatic dispersion, and should two solitons (for example at different wavelengths) collide, or pass through each other, they remain solitons. The performance of a soliton system may be readily calculated from the signal to noise ratio, and a perturbation analysis of the pulse properties, such as center frequency and arrival time. The most famous effect, the Gordon-Haus effect [82] results from the interaction between individual solitons, amplified spontaneous emission noise and dispersion. In brief, the noise is absorbed into the soliton, but changes its central frequency. Coupled with chromatic dispersion, this frequency jitter results in an arrival time jitter, in turn resulting in the potential for detection errors (pulse arrives in the wrong time slot). Various conditions must be satisfied to guarantee soliton transmission relating to amplifier spacing (should be as short as practical) and pulse duty cycle (should be as low as practical to minimize inter-soliton interaction) amongst others. The impact of these conditions was often illustrated in so called soliton design diagrams. Actual performance limits were readily calculated, as shown in Figure 5, from perturbation analysis from the interaction between soliton and noise [82], and from other nonlinear effects such as the Raman effect [83] and the acoustooptic effect [84], with the latter two of increasing importance for high symbol rate systems.

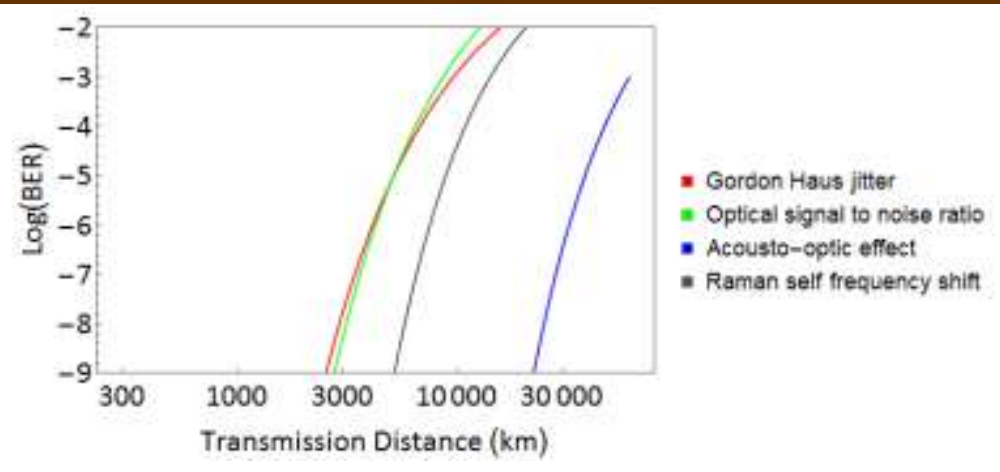

Figure 5: Analytically predicted performance of a $33 \mathrm{Gbit} / \mathrm{s}$ on-off keyed soliton transmission system with an amplifier spacing of $34 \mathrm{~km}\left(50 \mu \mathrm{m}^{2}, 0.2 \mathrm{~dB} / \mathrm{km}\right.$ loss $)$, and $7.7 \mathrm{dBb}$ noise figure amplifiers for uniform dispersion fiber. Dispersion optimised to $16 \mathrm{fs} / \mathrm{nm} / \mathrm{km}$ and pulse width to $1.3 \mathrm{ps}$. Shows analytically predicted bit error rate for signal to noise ratio (green, including spontaneous-spontaneous beat noise), jitter from the Gordon-Haus effect (red), Raman selffrequency shift (purple), and the acousto-optic effect (blue). Adapted from [32].

Figure 5 illustrates analytically predicted performance for a $33 \mathrm{Gbit} / \mathrm{s}$ on-off keyed soliton transmission system, using fiber and amplifier parameters typically experienced during the heyday of soliton transmission research. Analytically, it is possible to fine tune the dispersion parameter and pulse width, higher dispersion or shorter pulse widths resulting in higher soliton launch powers (so that the increased dispersion is balanced by increased self-phase modulation). Whilst this improved the signal-to-noise ratio, the efficiency to which dispersion converts frequency noise into jitter implies that the net effect of increasing dispersion is to greatly increased jitter. On the other side, reduced dispersion results in a reduced launch power and increased impact from amplified spontaneous emission noise. In order to achieve a transmission distance exceeding a few thousand $\mathrm{km}$, it is necessary to control the dispersion to an accuracy of better than $0.02 \mathrm{ps} / \mathrm{nm} / \mathrm{km}$, and consequently no experimental results have matched this performance prediction. The acousto-optic and Raman effects are even more susceptible to dispersion and transmission results over such distances were strongly jitter limited, even at $10 \mathrm{Gbit} / \mathrm{s}$. 
Due to the strict requirement to achieve specific dispersion coefficients described above, in order to optimize performance at particular bit rates, path averaged dispersion was controlled by the addition of a second, high dispersion, fiber [75]. Including the second fiber was initially carried out simply to tune the mean dispersion, and was basically an experimental convenience. However, whilst many aspects of these experiments agreed with analytical predictions and numerical simulations, it was observed that such periodic dispersion management led to an increase in the optimum soliton power above the theoretical values suggested for uniform dispersion. This increase in soliton power arose because the increased pulse dispersion reduced the effectiveness of self-phase modulation. The increased signal power reduced the impact of ASE, allowing for operation with lower path averaged dispersion, larger pulse width, and consequently reduced jitter [74,76]. Dispersion management led to a significant increase in transmission reach, as illustrated by comparing the case shown in Figure 6 with that in Figure 5. Not only does this result in lower Gordon Haus jitter, but it further reduces the impact of the acousto-optic and Raman effects. Such optimization enables transoceanic transmission to be envisioned, and indeed ultra-long haul transmission results were reported for soliton based systems [85], and a close agreement with theoretical performance limits was achieved.

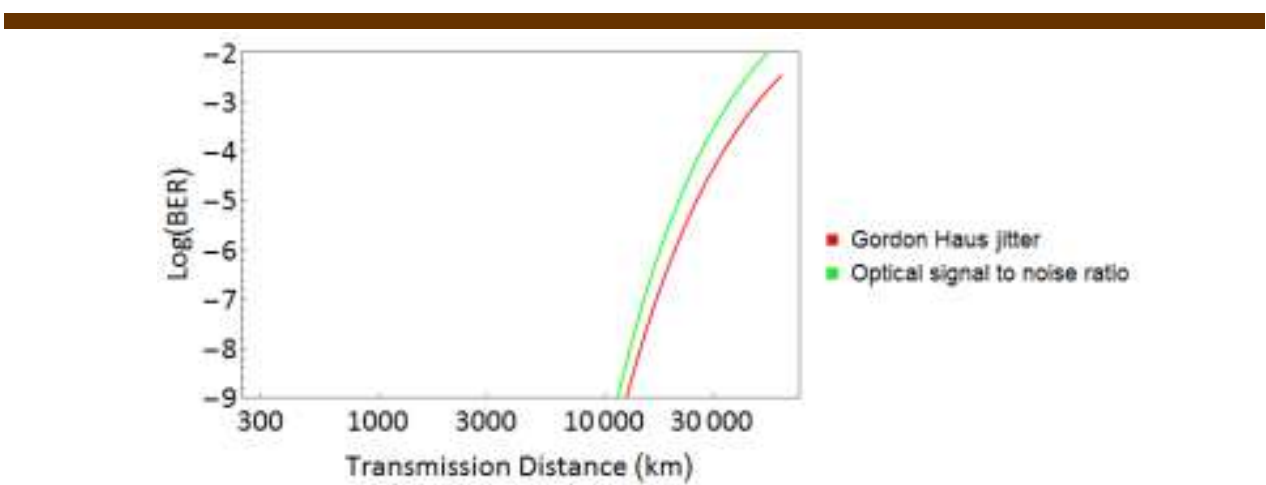

Figure 6: Analytically predicted performance of a $33 \mathrm{Gbit} / \mathrm{s}$ on-off keyed soliton transmission system with an amplifier spacing of $34 \mathrm{~km}\left(50 \mu \mathrm{m}^{2}, 0.2 \mathrm{~dB} / \mathrm{km}\right.$ loss $)$, and $7.7 \mathrm{~dB}$ noise figure amplifiers for a dispersion managed link. Dispersion profile optimised to $-1 \mathrm{ps} / \mathrm{nm} / \mathrm{km}$ for $32 \mathrm{~km}$ of fiber followed by $2 \mathrm{~km}$ of $16 \mathrm{ps} / \mathrm{nm} / \mathrm{km}$ fiber. Pulse width optimised to $3.1 \mathrm{ps}$. Shows analytically predicted bit error rate for signal to noise ratio (green, including spontaneousspontaneous beat noise), and jitter from the Gordon-Haus effect (red). Raman self-frequency shift and the acousto-optic effect are negligible in this case. Adapted from [32].

Soliton system performance was further improved by the addition of partially regenerative functions in the transmission line which exploited the natural stability of a soliton pulse against weak perturbations to restrict the growth of frequency jitter [86], negate its impact through the imposition of frequency chirp [87] or phase conjugation [88], or to drag the soliton amplitudes towards the ideal position through amplitude modulation [89]. The concepts of dispersion managed solitons were successfully used to design commercially deployed transmission systems [90] and analytical predictions of system performance were readily available and highly reliable. The advent of high spectral efficiency transmission systems resulted in a sharp decline in interest in soliton transmission systems which required spectrally inefficient picosecond pulses for optimum performance. The study of soliton transmission systems revealed for the first time the following lessons;

- Simple but accurate expressions to predict the performance of a nonlinear transmission system were possible.

- $\quad$ System designs may gain a degree of benefit by taking into account the nonlinearity.

- $\quad$ Performance is ultimately limited by the interaction between signal and noise.

- $\quad$ The nonlinear interaction between signal and noise may itself be addressed by introducing carefully designed elements distributed along the transmission link.

- $\quad$ For long distance systems with large spectra (short pulses in the soliton context) acoustooptic and Raman effects should not be neglected.

Whilst explicit research into soliton transmission systems is now rare, recent calculations have shown the benefit of multi-level phase modulated solitons $[91,92]$ and continued interest in dispersion management $[93,94]$ and control [95] of optical solitons. The broader lessons of soliton transmission systems are 
currently being revised through the generalized concept of the nonlinear Fourier transform [96], and calculations of potential performance limits are now under way [97].

\subsection{Wavelength division multiplexed systems}

To further increase the total system throughput, multiple signals may be multiplexed over the various available dimensions, including wavelength (frequency [18, 98]), polarization, phase, and space [99]. Given that the Shannon limit predicts throughputs proportional to $N_{c h} B \log _{2}(1+s n r)$ [100] one would expect that increasing the channel bandwidth, through for example wavelength division multiplexing (WDM), would be an easier route than increasing the signal power in order to enhance the signal to noise ratio. Indeed, this has historically been the case, with wavelength division multiplexing, quadrature multiplexing and polarization multiplexing all preceding the use of multiple amplitude levels in core networks (this has not been the case for short reach links, where the complexity (cost) has outweighed the theoretical performance benefits). To analyze the nonlinear interaction between signals the carrier envelope $u(z, t)$ in Equation 10 is usually replaced with the sum over three potentially different wavelength signals $u=u_{i}+u_{j}+u_{k}$, giving rise to nonlinear interference terms governed by the second terms on the right hand side. If all three terms are identical $(i=j=k)$ we have contributions commonly referred to as self-phase modulation (SPM) and the impact of nonlinearity is as described in section 3.1, if two of the terms are identical (say $i=j \neq k$ ) part of the nonlinear term is proportional to the intensity of the interfering channel, given effects known as cross-phase modulation (XPM). Finally if all three terms are unique $(i \neq j \neq k)$ the effects are known as four-wave mixing (FWM) [101]. Degeneracy factors $(D)$ often arise from mathematically identical permutations of the fields in the description of certain phenomena, such as double strength of cross phase modulation $(D=6)$ and self-phase modulation $(D=3)$. It is important to note that all of the different classes of nonlinear penalty described by equation 10 have the same origin, and provided care is taken with both degeneracy (the number of permutations of any given combination of signals) and the finite spectral width of real signals, the impact of nonlinearity may be calculated using the most general approach, four-wave mixing. Taking this approach, we find for an optically amplified link, that the nonlinearly generated field component at $\omega_{t}=\omega_{i}+\omega_{j}-\omega_{k}$ is given by [102-104]

$$
u_{t}=\left(2 u_{i} u_{j} u_{k}^{*} \gamma e^{j \Delta \beta N_{a} L}\right)\left(\frac{1-e^{\alpha L} e^{-j \Delta \beta L}}{\alpha+j \Delta \beta}\right)\left(\frac{\operatorname{Sin}\left(N_{a} \Delta \beta L / 2\right)}{\operatorname{Sin}(\Delta \beta L / 2)}\right)
$$

Where the phase matching parameter, $\Delta \beta$, is given by

$$
\Delta \beta=-\beta^{\prime \prime}\left(\omega_{i}-\omega_{k}\right)\left(\omega_{j}-\omega_{k}\right)
$$

and where $u_{i, j}$ are the optical field envelops of the mixing components located at frequencies $w_{i j, k}$, and $u_{t}$ is the resulted FWM optical field envelop spectrally located at $\omega_{t}=\omega_{i}+\omega_{j}-\omega_{k}$. In equation 16 , the first term represents the overall scale of the generated signal and is dominated by the cube of the optical field amplitude, and the fiber nonlinearity. This term is often expressed with a degeneracy factor D which counts the number of permutations of the fields contributing to a given nonlinear effect, such as cross phase modulation $(\mathrm{D}=3)$. The second term represents the effect of phase matching of a single span which is dominated by the dispersion and loss effective lengths of the fiber [105], and the final term represents the quasi-phase matching effect of the cascaded amplifier chain [106]. This last term is expressed for a uniform system with identical spans, and is readily verified experimentally [107]. 


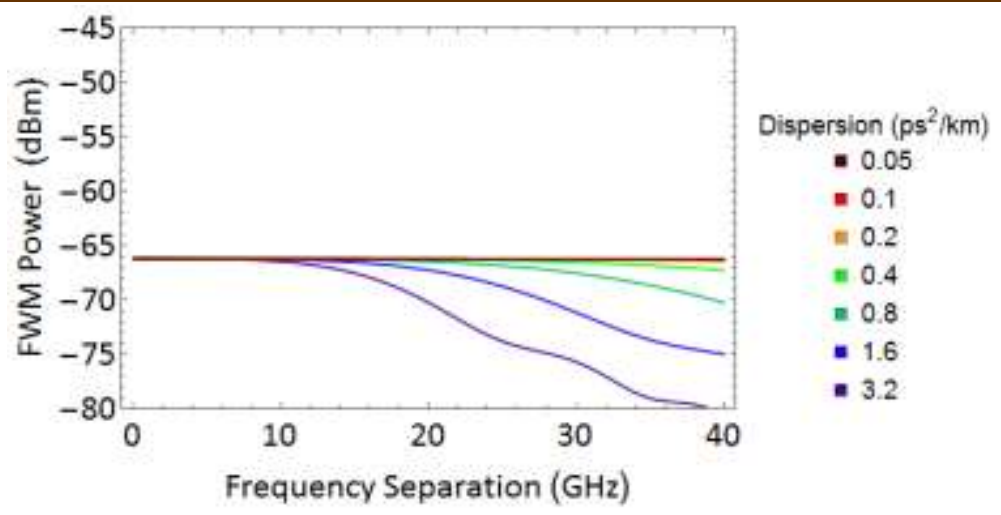

Figure 7. FWM power as frequency separation between two frequency components for single $65 \mathrm{~km}$ span of fiber assuming a nonlinear coefficient of $1.4 / \mathrm{W} / \mathrm{km}$, loss of $0.2 \mathrm{~dB} / \mathrm{km}$ with signal launch power of $-10 \mathrm{dBm}$, amplification exactly overcome span loss, and dispersion coefficients, $\beta$ ", of $3.2,1.6,0.8,0.4,0.2,0.1$ and $0.05 \mathrm{ps}^{2} / \mathrm{km}$ (purple to mauve).

Figures 7 and 8 show the FWM power $\left|\mathrm{ut}_{\mathrm{t}}\right|^{2}$ (resulted by the end of optical transmission system) as a function of frequency separation between two mixing optical components. It can be seen that for a single span system (figure 7) there is no effect of phase mismatch resulted from the third term because it is equal to 1 , and the second term of equation 16 will start raising phase mismatch (as fiber dispersion increases) between the mixing components which result degradation of the FWM power as the frequency separation increases. For multi-span system (figure 8), we can see that the third component of equation 16 will result higher FWM power ( $20 \mathrm{~dB}$ in the figure) at strongly phase matched mixing components (low frequency separation) since the contribution to the detected power arising from the third term will be proportional to $\mathrm{Na}^{2}$, at weakly phase matched mixing components (higher frequency separation) the third term ratio of equation 16 will start showing an oscillation in FWM that depends on the dispersion length and the number of spans in the system.

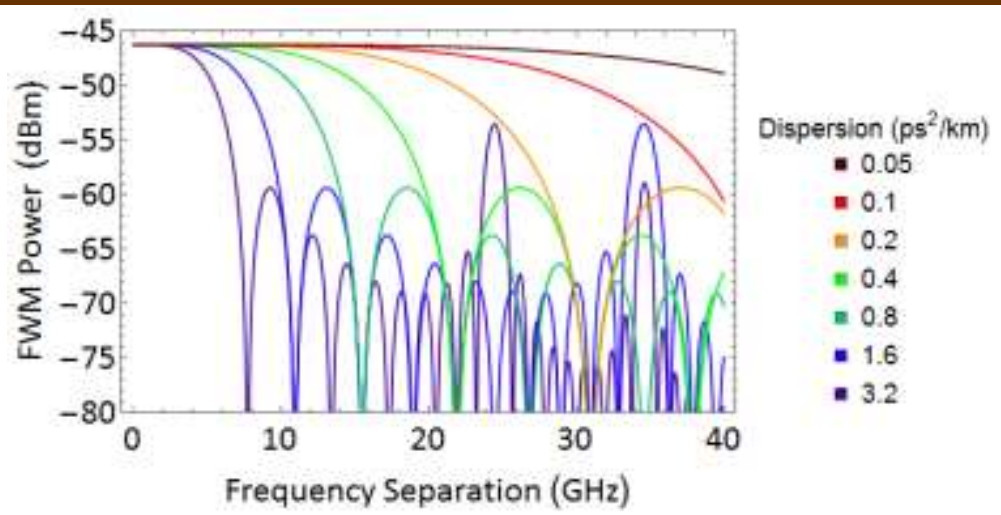

Figure 8. FWM power as frequency separation between two frequency components for a link comprising ten $65 \mathrm{~km}$ spans of fiber assuming a nonlinear coefficient of $1.4 / \mathrm{W} / \mathrm{km}$, loss of 0.2 $\mathrm{dB} / \mathrm{km}$ with signal launch power of $-10 \mathrm{dBm}$, amplification exactly overcome span loss and dispersion coefficients, $\beta^{\prime \prime}$, of $3.2,1.6,0.8,0.4,0.2,0.1$ and $0.05 \mathrm{ps}^{2} / \mathrm{km}$ (purple to mauve).

For any given system, equation 16 can be used to vectorially calculate the magnitude of any unwanted power which may be coherently added to the signal to allow for the calculation of eye closure or signal to noise ratio penalties. For a uniform dispersion system, the predilection for low dispersion to minimize intra channel effects observed in section 3.2 tends to enhance inter-channel four wave mixing by extending the phase matching bandwidths to ever higher frequency separations. This rapidly increased the impetus for dispersion managed systems where the objectives are to maintain high local dispersion to minimize the nonlinear interactions in each span (second term of equation 16), a low path average 
dispersion to minimize pulse broadening and single channel effects (see section 3.2). Furthermore, if the accumulated dispersion returns to zero after each period of the dispersion map, four wave mixing products from each map section add up coherently, giving another source of quasi phase matched four wave mixing enhancement, as was seem for uniform dispersion systems in the (third term of equation 16) [107]. Consequently an additional parameter, the residual dispersion per span (or per map period if longer than one span) was introduced. The optimum value was a trade-off between large quasi phase matching which enhances inter-channel nonlinearities (favoring large residual dispersion) and total accumulated dispersion which increases the intra-channel peak to average power ratio (favoring low residual dispersion). For the general case, contributions from each amplified span must be summed vectorally $[108,109]$, but considerable insight may be gained from various special cases, allowing for the map shape [110] and length [111] to be tailored with a view to minimizing resonances [112]. Excellent experimental validation has been observed for a variety of cases involving periodically varying parameters $[113,114]$, with the minimum four wave mixing powers observed for strong aperiodic maps. In $[109,115]$ a simple approximation was proposed for short periods maps (where two or more fibers were used within each span);

$$
u_{t} \approx\left(u_{i} u_{j} u_{k}^{*} \gamma_{1} e^{j \widetilde{\Delta \beta} L / 2}\right)\left(\frac{1-e^{\alpha_{1} L_{1} e^{-j \Delta \beta_{1} L_{1}}}}{\alpha_{1}+j \Delta \beta_{1}}\right)\left(\frac{\operatorname{Sin}\left(N_{a} \widetilde{\Delta \beta} L / 2\right)}{\operatorname{Sin}(\widetilde{\Delta \beta} L / 2)}\right)
$$

where the numerical subscript refers to the parameter of the indicated fiber, and the path weighted phase matching factor is given by

$$
\widetilde{\Delta \beta}=-\frac{\beta_{1}^{\prime \prime} L_{1}+\beta_{2}^{\prime \prime} L_{2}}{L_{1}+L_{2}}\left(\omega_{i}-\omega_{k}\right)\left(\omega_{j}-\omega_{k}\right)
$$

Figure 9 shows the FWM power $\left|\mathrm{ut}_{\mathrm{t}}\right|^{2}$ at the end of a dispersion managed optical transmission system as a function of frequency separation between two mixing optical components. For a single span system (not shown), there is no significant effect resulting from the addition of a piece of DCF after transmission because the signal power at the input of the DCF may be made arbitrarily low $\left(\mathrm{e}^{\alpha_{1} \mathrm{~L}_{1}}\right.$ in the second term of equation 18 if the dispersion compensating fiber forms part of the span) so there are very small FWM power generated in the DCF. For a multi-span system however, the DCF impacts the relative phases of the interacting signals and so the dispersion compensation ratio has a significant effect on the FWM power oscillation. We can see that full compensation of dispersion will cancel the phase mismatch resulting from the third term leaving a mixing product who's power is proportional to $\mathrm{N}_{\mathrm{a}}{ }^{2}$, suggesting that a $100 \%$ dispersion compensation ratio should be avoided.

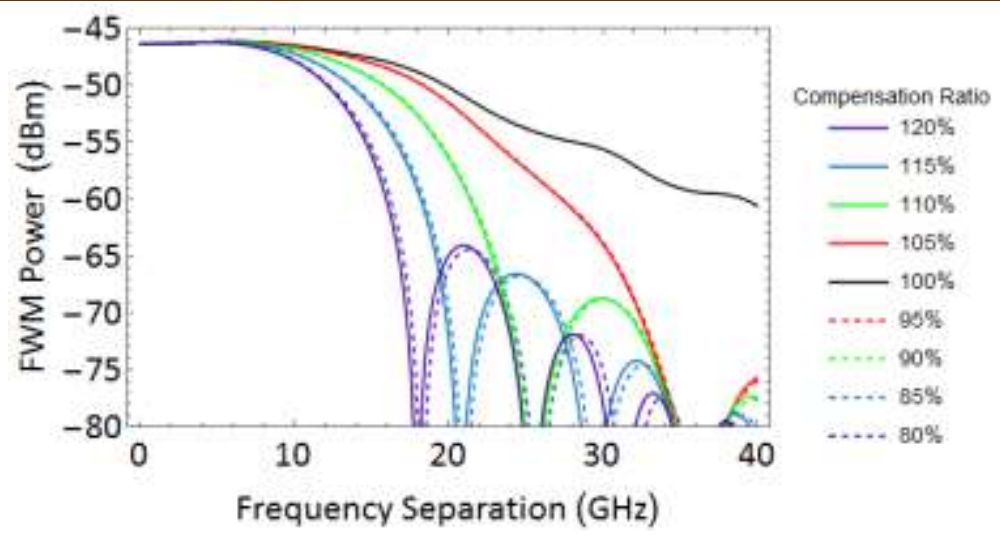

Figure 9: FWM power in a dispersion managed system as a function of separation between two frequency components for a system with ten spans. Each span considered in the calculation had a $65 \mathrm{~km}$ transmission fiber with a nonlinear coefficient of $1.4(\mathrm{~W} \cdot \mathrm{km})^{-1}$, a loss of $0.2 \mathrm{~dB} / \mathrm{km}$, a dispersion coefficient of $3.2 \mathrm{ps}^{2} / \mathrm{km}$, a signal launch power of $-10 \mathrm{dBm} \mathrm{dBm}$, an amplifier exactly compensating all span losses, and a dispersion compensating fiber with a length depending on the dispersion compensation percentage, nonlinearity coefficient of 1.4(W.km) ${ }^{-1}$, a loss of $0.2 \mathrm{~dB} / \mathrm{km}$, and a dispersion coefficient of $-40 \mathrm{ps}^{2} / \mathrm{km}$. Colors represent the dispersion compensation ratio (total dispersion of dispersion compensating fiber divided by total dispersion of transmission fiber) : solid lines; purple, 80\%; blue, $85 \%$; green $90 \%$; red, 95\%; black $100 \%$ and dashed lines; purple, $120 \%$; blue, $115 \%$; green $110 \%$; red, $105 \%$. 
This analytical approach proved to be accurate provided that $L_{1}>>L_{2}$, as were other related approaches which considered only a fraction of the four-wave mixing terms, such as cross phase modulation [116] in order to simplify the derivation and/or the exposition. In these cases, the generated four wave mixing terms were considered as an additional noise source, affecting both ones and zeros, in equation 5 . Mixing products arising from the same combination of channels were added vectorially due to their correlation, and those from independent combinations of channels were added incoherently as independent random variables. Proposals to calculate system performance using these quasi phase matched integrals were made [117]. However, for small channel counts, full numerical simulation of equation-10 was possible, minimizing the risk of assumptions being breached or effects being neglected whilst for large channel counts the complexity of the analytical calculations rapidly becomes unwieldy except for certain special cases. As a consequence of this, despite the availability of accurate but little known models based on these principles [118], system design of signal channel, wavelength division multiplexed and even dense wavelength division multiplexed systems continued to rely on numerical simulation $[119,120]$.

\section{Coherently Detected Systems}

We have seen how, throughout the history of optical communications, it has been possible to develop accurate analytical predictions of the system performance. These applied to single channel systems, with and without optical amplifiers, to soliton transmission systems, and to wavelength division multiplexed systems with dispersion management. However, such models rarely gained widespread acceptance and numerical simulations dominated system design. The widening use of coherent detection, along with digital signal processing, appears to have changed the emphasis significantly with analytical predictions more frequently used. This is partly due to a realization of the simplicity of the model [118], but also the availability of digital signal processing to eliminate the impact of component imperfections, greatly improving the model accuracy. Before considering the model, and its use to predict the maximum potential performance of a communication system, we review the basic properties of a coherent transmission system.

\subsection{Linear performance of a coherent transmission system.}

At a fundamental level, the inclusion of a local oscillator in the receiver of a communication system has two significant implications. Firstly, the beating between the local oscillator and the received field results in the generation of additional photocurrents proportional to the received signal amplitude, as opposed to solely the signal intensity. This gives ready access to two orthogonal field quadratures and both polarizations [121-123], allowing the data rate to be immediately quadrupled for the same symbol rate and number of amplitude levels. It also allows the possibility of applying signal processing functions which would be difficult in the optical domain, such as spectral filters with sharp roll offs [18,124-125] and adaptive all pass filters with long memory lengths enabling, for example, electronic compensation of chromatic dispersion $[126,127]$. A particularly important linear compensation function associated with digital coherent receivers is the compensation of substantial levels of polarization mode dispersion. Secondly, if the local oscillator power is sufficiently high, photocurrents proportional to the local oscillator intensity dominate, enhancing the receiver sensitivity (or improving the required optical signal to noise ratio) [124]. The wanted signal $I_{L S}$ and additional shot $\left(I_{L S h}\right)$ and beat noise $\left(I_{L a}\right)$ terms are [128];

$$
\begin{gathered}
I_{L S}^{2} \propto P_{L} P_{s} \\
I_{L s h}^{2} \propto P_{L} P_{e} B_{r} \\
I_{L a}^{2} \propto P_{L} P_{a} \frac{B_{r}}{B_{S}}
\end{gathered}
$$

where $P_{L}$ represents the local oscillator power. Further benefits are observed when balanced receivers are used, with signal-spontaneous and spontaneous-spontaneous beat noise appearing as common mode noise, which may be suppressed by a balanced receiver with appropriate characteristics [129]. In this case, any signal which does not originate from beating with the local oscillator is common to both arms of the balanced receiver and so is substantially cancelled when the arms are subtracted. For an ideal balanced receiver, all photocurrent terms in equation- 4 except that relating to thermal noise become zero. Thermal noise is, unfortunately, doubled for a balanced receiver. Following the approach of section 3.1 and assuming ideal balanced receivers, the received signal-to-noise ratio is given by;

$$
s n r_{C D}=\frac{(1+b d) \sqrt{I_{S L}}}{2 \sqrt{(1-b d)\left(I_{s a}+I_{a s h}+I_{a a}\right)+I_{s s h}+I_{L s h}+I_{L a}+(1+b d) I_{t h}}}
$$

where the detection parameter $b d$ is 0 for a single ended detector and 1 for a balanced detector. We assume henceforth an ideal intra-dyne balanced receiver with digital signal processing [130], and that the local oscillator power is sufficient to make the contribution of thermal and shot noise terms negligible. 


\subsection{Nonlinear signal to noise ratio in a coherent transmission system.}

In contrast to calculating the predicted performance of specific, cost reduced, transmission systems, in order to calculate fundamental performance limits, it is necessary to consider a fully optimized system. Fundamental theorems in communication push the system designer towards; maximizing the channel bandwidth rather than signal to noise ratio as system throughput scales linearly with bandwidth but only logarithmically with signal-to-noise ratio [100]. For memoryless systems employing matched filters to optimize the trade of between inter-symbol interference [131] and noise [132] is of significant benefit, whilst correlated signaling, which deliberately introduces controlled inter-symbol interference [133] or cancelation of intentionally induced inter-symbol interference by maximum likelihood sequence estimation [134] may be used to trade off total capacity and required signal to noise ratio. Modulation formats and coding should be optimized including bi-polar formats to maximize use of the transmitted energy and adapting the constellation to the almost [135] Gaussian noise of the linear optically amplified channel [136]. Having developed a strategy to optimize the per channel performance, it remains to fully exploit the available spectrum by adding additional channels. This is ideally performed using fundamentally orthogonal pulse shapes to minimize inter-channel interference [137] leading to concepts of orthogonal frequency division multiplexing (OFDM) and filter bank multi-carrier $[138,139]$ or using signals with almostrectangular spectra [140]. All of these techniques have been shown to increase the net throughput of an optical system in the ASE noise dominated regime, and whilst it has been argued that slight modifications may be beneficial for nonlinear channels [141], the general principles remain valid. In contrast to the early optically amplified systems described above, where the evolution of pulse parameters could be calculated analytically and used to provide predictions of maximum system performance, the nonlinear evolution of individual channels in such high spectral efficiency systems are difficult to state analytically. Furthermore, the ability to access the full field of the signal offered by coherent detection enables linear impairments to be compensated in the electrical domain (either digitally, or using appropriate analogue circuits) making the approach less valid. An alternative approach is needed for the analysis of such systems which identifies those changes to the received optical field which may not be simply compensated by linear filters in the electrical or digital domain. In order to establish the fundamental performance limit imposed by nonlinearity, rather than the limits of a specific configuration, the system of interest, shown as the top row in figure 10 below, is a wideband multiplex of many independent channels, each channel carrying polarization multiplexed signals with bipolar modulation formats which exploit both quadratures of the optical field. The signals are either spectrally shaped, or overlapping in order to maximize the number of transmitted symbols per unit bandwidth, and we assume that an optimized linear filter is used to compensate for any and all linear impairments. We assume all of this, together with the additional assumption that the transmitters and receivers are all independent of each other (that is, are unable to exchange information about the data they are processing) and are carrying independent data. If all linear impairments are compensated it has been proposed that we may treat the impact of nonlinearity as the generation of an independent nonlinear noise field (of field amplitude $u_{n l}$ ), which is detected along with the signal $\left(u_{t}\right)$ and ASE $\left(u_{a s e}\right)$ at the receiver where they mix with the local oscillator field $\left(u_{l o}\right)$, giving a total photocurrent proportional to;

$$
\boldsymbol{i}_{t} \propto\left|\boldsymbol{u}_{l o}+\boldsymbol{u}_{s}+\boldsymbol{u}_{a s e}+\boldsymbol{u}_{n l}\right|^{2}
$$

and assuming an ideal balanced receiver eliminating common mode noise (including beat products from fields arriving at the same input of the optical hybrid), and, as stated above, electronic compensation of any residual chromatic and polarization mode dispersion, the received electrical signal power receives an additional noise term proportional to the beat between the local oscillator and nonlinear noise $\boldsymbol{I}_{\boldsymbol{S n l}}^{\mathbf{2}}$

$$
I_{S n l}^{2} \propto P_{L} P_{N L} \frac{B_{r}}{B_{S}}
$$

giving a total signal to noise ratio, neglecting shot noise of:

$$
\operatorname{snr}=\frac{B_{S}}{B_{r}} \frac{P_{S}}{P_{a s e}+P_{N L}}
$$

Where $P_{N L}$ represents the nonlinear noise power generated by the system in the bandwidth $B S$ (note that for a WDM system the total system bandwidth is typically greater than the transmitter bandwidth $B_{T x}$ by the number of channels. And is developed further in equation 20. This approach, first proposed in the 1990's [118] and revisited at the turn of the century [142-144] represents a significant departure from the previous, pulse shape dependent, approach and instead relies solely on the power spectral density of the signal. Separating the nonlinear interaction as a noise term relies on a system configuration where the spectral components interacting are random, which is achieved for low symbol rate signals such as OFDM, and signals which experience significant dispersion within the nonlinear effective length. 
When incorporated in Shannon and Hartley's eponymous formula $C / B=\log _{2}(1+s n r)$, equation 24 leads to what has been known as the nonlinear Shannon limit, although it does not represent a true limit in the general sense intended by Shannon, rather a lower bound governed by the choices made in designing the nonlinear transmission system, and the assumption of memoryless signal processing. The term limit refers to the fact that for any given system design there is an optimum launch power at which the signal to noise ratio, and thus data information spectral density, reaches its maximum. A popular approach to calculating this limit is to analytically integrate the nonlinear Schrödinger equation (equation $10)$ over distance (equation 18) and frequency [118, 140, 142, 145-146] subject to certain simplifying assumptions. In addition to those already listed above, these predominantly include the assumption that for the majority of the transmission link, all spectral components may be considered independentrandom variables and that the contributions from each span add with random phase. Alternative approaches based on time domain response functions or perturbation approaches give similar results (see section 4.3). In all cases, it is further assumed that the neteffect of nonlinearity is small. In the case of the frequency domain integration approach, this corresponds to the joint assumptions of negligible pump depletion and the absence of higher order four wave mixing (between at least one nonlinear mixing product and the signals). In the case of the perturbation approaches, this corresponds to a simplified first order model. It is reassuring that each approach gives the same result in the region where this basket of assumptions holds.

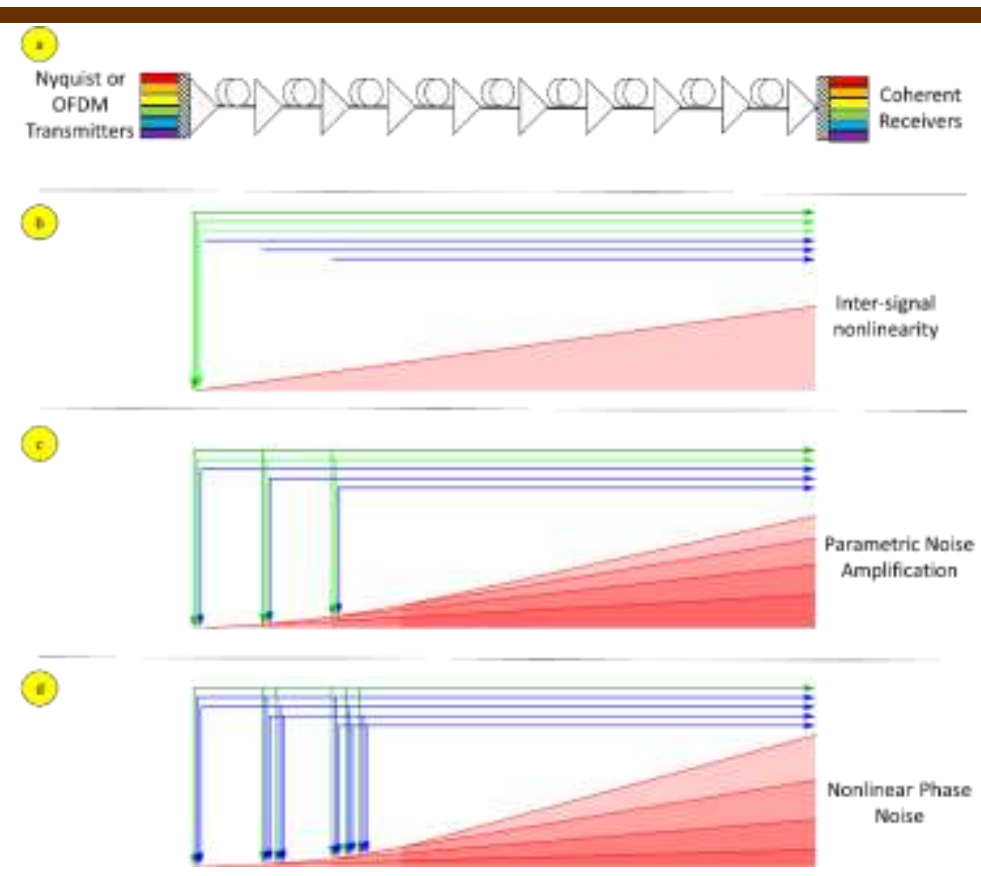

Figure 10: Interacting fields contributing to total nonlinear noise power, showing input signal fields (green horizontal lines) and noise fields from each amplifier (blue horizontal lines), the start of interactions between them (vertical arrows) and the nonlinearly generated signals (red shaded triangles). Each row shows a different interaction, specifically; inter signal interaction (b), parametrically amplified noise (c), and nonlinear phase noise (d). Nonlinear noise is not shown for simplicity.

A typical transmission system is shown in Figure 10a and comprises a number of independent closely spaced transmitters, ideally with flat power spectral densities. Such signals include Nyquist WDM signals [147], OFDM signals [148] or all-optical OFDM signals [149] or super-channels [150]. The signals are transmitted over a link comprising multiple spans of fiber, with periodic amplification, either in the form of discrete amplifiers, such as Erbium doped fiber amplifiers, or distributed amplifiers, commonly based on the Raman Effect. At the receiver each channel (or super-channel) is detected independently. Figure $10 \mathrm{~b}$ illustrates the two most dominant effect which impact the signal-to-noise ratio. The horizontal green lines represent the signal field, the three copies representing three frequency components involved in four-wave mixing. These signals are present at the input to the signal, and their nonlinear interaction may be determined using equation 16 (or estimated using equation 18 for a dispersion managed system). The 
start of this interaction is indicated by the greed vertical lines at the system input. The growth of the nonlinear mixing product is illustrated by the red shaded area, and the noise power grows linearly with distance. The second limiting feature is that each optical amplifier also contributes amplified spontaneous emission, represented by the blue horizontal lines (note only contributions from the first three amplifiers are shown). At the receiver, the signal, noise and nonlinear product fields are simultaneously detected in the coherent receivers.

Of course, once the amplified spontaneous emission is present, it may also interact nonlinearly with the signals. For example, Figure 10c illustrates the process where two signal field components interact with one noise component, with horizontal lines again representing the propagation of each field, vertical lines the initiation of a nonlinear interaction, and the shaded red areas the resulting nonlinear interaction (equations 16 or 18). This figure shows that the noise originating from each amplifier in the link independently interacts with the signal. The interaction between the signals and the noise from each amplifier still grows linearly, however, as a new interaction is added at each amplifier site, the total noise field from this interaction grows approximately quadratically. Of course, different permutations of signal and noise fields are possible, and figure $10 \mathrm{~d}$ shows the interaction between one signal field and two noise fields. As the number of combinations of interacting noise fields increases with the addition of each amplifier (schematically shown by the number of vertical lines, each triplet corresponding to a different interaction), the power originating from this interaction tends to scale cubically. Nonlinear interactions between three noise field components are also possible, but are not shown in figure 10 .

The fundamental assumption here is that all linear fields (signals and noise) present at the input of any and all spans interact via the nonlinearity for the remaining length of the transmission system, and that the nonlinear noise products produced in this way are statistically independent. In order to calculate the total noise power, the nonlinear noise field generated by all possible combinations of signal and noise frequencies are integrated over the signal spectrum, again assuming independence of the initial frequency components. A detailed derivation of the integration of equation 16 to give the nonlinear noise power at a particular frequency may be found in [145] for a dispersion managed system (and thus indirectly of equation 18 Note that for notational simplicity the authors express their results in spectral densities as opposed to signal powers. Following this approach for a system with uniformly spaced amplifiers, and setting $P_{N L}=D_{N L} B_{R x}$ and $P_{a}=N_{a} D_{a} B_{R x}$ we find that the nonlinear noise power may be calculated from its spectral density $D_{N L}$ which I in turn determined from that of the signal $D_{S}$ using;

$$
D_{N L}=\zeta_{0} \Gamma D_{S}^{3}+3 \zeta_{1} \Gamma D_{S}^{2} D_{a}+3 \zeta_{2} \Gamma D_{S} D_{a}^{2}+\zeta_{3} \Gamma D_{a}^{3}
$$

Where the length scaling parameters, $\zeta_{\mathrm{i}}$ are

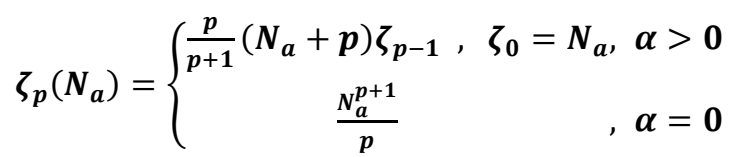

And the aggregated nonlinear scaling factor, $\Gamma$ is given by;

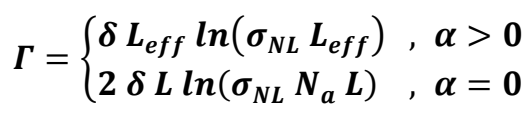

and where and $L_{e f f}$ represents the conventional effective length of the fiber, $\sigma_{N L}=2 \pi^{2}\left|\beta_{2}\right| B_{S}^{2}$ the inverse of the dispersion length of a pulse with spectral width $B_{S}$, and $\delta=\gamma^{2} /\left(\pi / \beta_{2} \mid\right)$ a measure of the relative impact of dispersion on nonlinearity. The index $\mathrm{p}$ indicates the nonlinear process and is equal to the number of four wave mixing components which are taken from the amplified spontaneous emission noise. Equation 27 could equally be written as (for $\alpha>0) \Gamma=\delta L_{e f f} \ln \left(B_{S}^{2} / f_{w}^{2}\right.$ ) where we define $f_{w}^{-2}=2 \pi^{2}\left|\beta_{2}\right| L_{e f f}$ and represents the bandwidth of the first lobe of the four-wave mixing efficiency characteristic (figure 8). This approach implicitly assumes that the total signal bandwidth exceeds the four-wave mixing efficiency bandwidth $\left(\mathrm{B}_{\mathrm{S}}>>\mathrm{B}_{0}\right)$. The degeneracy factor of 3 in equation 25 arises from the interchangeability of two signal and one noise component in the four wave mixing four wave mixing process $[151,152]$ (derived from equation 22) whilst the length scaling of equation 26 is found by summing the contributions from all signals and noise sources (each optical amplifier) independently, each possible triplet propagating over any remaining transmission fiber. The different terms in equation 25 represent inter-signal interaction (or four wave mixing), parametric noise amplification [42-44, 153-154], nonlinear phase noise (also known as the Gordon Mollenauer effect [155]), and what we shall term nonlinear noise [142] respectively. Note that this naming convention has not been adopted uniformly in the literature, with the phrases modulation instability and nonlinear phase noise used interchangeably with parametric noise amplification for the second term, with confusion between parametric noise amplification and nonlinear phase noise particular prevalent. For the majority of systems of moderate length and high signal-to-noise 
ratio $\left(D_{S} \gg D_{a}\right)$ these terms are arranged in order of decreasing magnitude and unless the system length is long [156] the second and subsequent terms may be neglected (see Figure 11). Note that strictly, the natural logarithms in equation 27 should be replaced with inverse hyperbolic sin, however it is often convenient to employ the logarithmic form when comparing systems. For a system which is not fully loaded, noise contributions the integrals need to take into account gaps between the signals [157], and approach which agrees with recent experiments [158]. For a totally uniform input spectrum, gain flat amplifiers (and noise) and wavelength independent fiber loss, these noise spectral densities (with the exception of nonlinear noise) are approximately uniform within the signal band, have a transition region with a width in the region of $B_{0}$ and are zero elsewhere. Nonlinear noise is generated throughout the amplified spontaneous emission noise bandwidth.

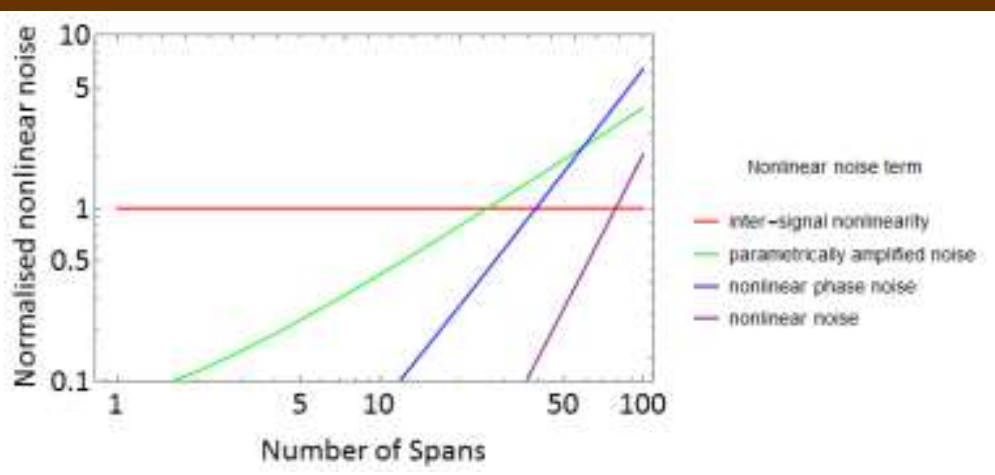

Figure 11: Relative impact of nonlinear interactions involving amplified spontaneous emission, showing normalised inter signal (red), parametrically amplified noise (green), nonlinear phase noise (blue) and nonlinear noise (purple) for a $16 \mathrm{~dB}$ optical signal to noise ratio after one amplifier.

Consider a dispersion managed system with dispersion compensators located at the amplifier sites, for example, at the mid stage of each amplifier. Assume that the dispersion compensators make no contribution to the nonlinear power spectral density, which is possible if they are filter based, or if the power propagating in a dispersion compensating fiber is sufficiently low. In this case, it can be readily shown that $\zeta_{0}$ becomes [145]

$$
\zeta_{0-D M}=\frac{2 e^{-\alpha L \epsilon}\left(N_{a} \operatorname{Sinh}(\alpha L \epsilon)+e^{-\alpha N_{a} L \epsilon_{-1}}\right)}{\left(e^{-\alpha L \epsilon_{-1}}\right)^{2}}, \alpha>0
$$

Where $\varepsilon$ represents the ratio of the residual chromatic dispersion per span to the dispersion of the transmission fiber. Closed form expressions also exists for the other $\zeta_{p-D M}$ but are rather cumbersome. Note that equation 26 is in fact the limit of equation 28 as $\varepsilon$ tends to zero, and that it is independent of the parameters of the signal. The resultant enhancement $\left(\zeta_{p-D M}>1\right)$ of the nonlinear noise spectral density increases the nonlinear noise, and so reduces the maximum system performance. The enhancement factor is often referred to as the phase array enhancement since it arises from periodically changing the relative phases of the nonlinearly interacting signals rather than from adding new nonlinear signals within the compensators themselves. Inspired by experimental observations of slightly supra linear growth with distance $[154,159]$, as an alternative to equation 28 , it has been heuristically proposed to simply raise the span length in equation 26 to the power of $\left(1+\varepsilon^{\prime}\right)$ where $\varepsilon^{\prime}<<1$. We refer to this approach as the power law approximation.

The enhancement factors resulting from Equation 28 are illustrated in Figure 12 for dispersion managed and unmanaged systems based on standard single mode fiber. This clearly shows a significantly greater enhancement of the nonlinearity for the dispersion managed case. There is also a clear enhancement associated with a shorter amplifier spacing. Both of these observations are consistent with the fundamental evolution of the four-wave mixing products detailed in equations 16 and 18. The fractional power law behavior, observed experimentally occurs for short distances, shows good agreement with the exact calculation below around 10 spans. Beyond this level the actual enhancement factor saturates to a constant value, whilst the power law approximation continues to grow, reducing the accuracy of predictions based on this approximation. It is interesting to note that beyond this point not only does the enhancement factor saturate (figure 12), one expects to begin to observe the impact of nonlinear interactions between the signal and noise fields (figure 11). The smooth transition between phased array 
enhancement and nonlinear interaction between signal and noise results in a continuation of the super linear growth of nonlinear noise. This extension of the super linear growth region may give the appearance of extending the validity of the heuristic power law approach. In actual fact, for short span lengths the nonlinear noise power grows super linearly due to the phase array effect, whilst at long span lengths it grows super linearly due to the interaction between signal and noise. Simple closed form expressions (equations 25 and 28) for both of these effects have been presented.

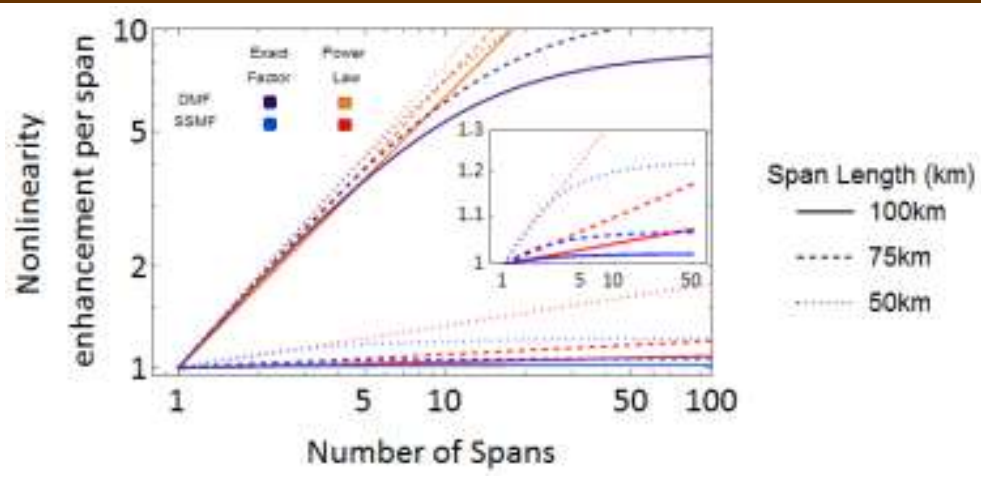

Figure 12: Relative impact of the phase array enhancement for links comprising standard single mode fibers with uniform dispersion (red, blue) or dispersion compensated single mode fiber with 95\% compensation (orange, purple). Links have either 100, 75 or $50 \mathrm{~km}$ amplifier spacing (solid, dashed and dotted respectively) and analytical results are shown for the exact phase array enhancement factor of equation 28 (blue, purple) and for the fractional power law approximation (red, orange). The inset shows a zoom of the same data for the dispersion unmanaged case.

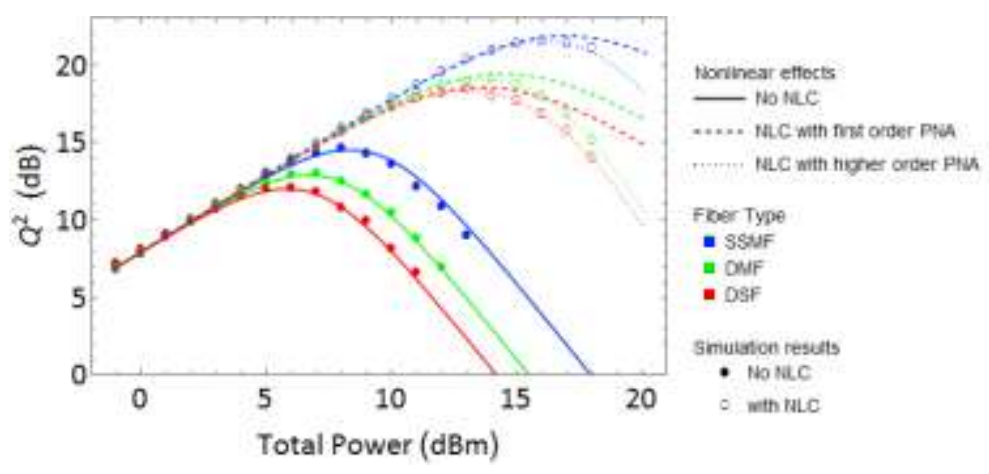

Figure 13: Simulated and predicted performance of lumped system with amplifier noise figure of $6 \mathrm{~dB}$ and passing 8 channel, 28 Gbaud PM-QPSK Nyquist WDM system over 12 spans of $100 \mathrm{~km}$ of fiber, either uncompensated standard single mode fiber (16 ps $/ \mathrm{nm} / \mathrm{km}$, blue), nonzero dispersion shifted fiber (4 ps/nm/ $\mathrm{km}$, red) or a dispersion managed system $(100 \mathrm{~km}$ standard single mode fiber between the amplifiers) with ideal slope compensation located at the mid stage of each amplifier and compensating 95\% of the dispersion of each span (green). Filled dots, simulation results with electronic dispersion compensation; open dots, simulation results with nonlinearity compensation of the full bandwidth of the total optical field. Solid lines theoretical predictions without nonlinearity compensation; dashed lines, theoretical predictions with digital back propagation considering only first order signal-noise interaction; dotted lines, theoretical predictions with digital back propagation considering both the first and second order signal-noise interaction. 
To verify the accuracy of the calculation of nonlinear noise, the solid lines and filled symbols of figure 13 and 14 show the simulated and predicted (with first and second order nonlinear interactions between signal and noise) nonlinear thresholds (figure 13, at $1200 \mathrm{~km}$ ) and distance evolution (figure 14, at the optimum power found at $1200 \mathrm{~km}$ ) of an eight channel 28 Gbaud PM-QPSK system for three common dispersion maps, uncompensated based on standard fiber, nonzero dispersion shifted fiber, and dispersion managed using slope compensating fiber with a residual dispersion per span of $5 \%$ of the standard fiber dispersion. It can be seen from the figure that lower accumulated chromatic dispersion in the link will lead to a degradation in system performance both for the case of receiver signal processing which compensates for nonlinearity [160] and in the case of receiver signal processing which only compensates for linear impairment [126]. Dashed and dotted lines illustrate the performance if the intersignal nonlinearity is compensated, and will be discussed more fully in section 5 .

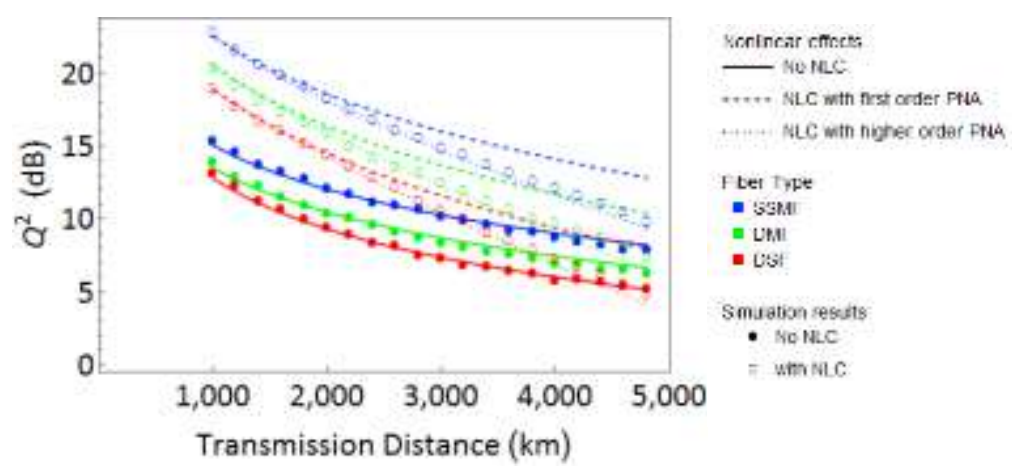

Figure 14: As figure 13, but as a function of transmission distance with signal power optimized at $1,200 \mathrm{~km}$.

Neglecting the impact of nonlinear interaction between signal and noise and of dispersion management, simple expressions may be derived to predict the optimum launch power spectral density and the optimum signal to noise ratio, which are given by $[145,161]$

$$
D_{o p t}=\sqrt[3]{\frac{D_{a}}{2 \Gamma}}
$$

and

$$
s n r_{o p t}=\frac{2}{3 N_{a}} \sqrt[3]{\frac{1}{2 \Gamma D_{a}^{2}}}
$$

With equation 30 readily rearranged to give a maximum reach. Note that the optimum signal power is predicted to be independent of distance for this ideal system limited only by the linear accumulation of amplified spontaneous emission and nonlinear interactions between different components of the signal, and is typically a few tens of $\mathrm{mW} / \mathrm{THz}$. To take into account dispersion management, both equations 29 and 30 should be multiplied by $\sqrt[3]{N_{a} / \zeta_{0}}$. A closed form expression also exists if all four terms of equation 25 are considered, however, a compact representation is not possible. Note that the assumptions thateach frequency component of the signal may be considered an independent random variable inherent in the derivation above break down if the system length is short [162] and for low cardinality modulation formats, such as, binary phase shift keying (BPSK) [163] and quadrature phase shift keying (QPSK) [164]. In these circumstances, strong correlations within the first lobe of the four-wave-mixing efficiency curve (equation 18) occur and the generated mixing products become more deterministic and more strongly correlated from symbol to symbol. These features allow for some of the nonlinear impairments to be partially compensated by linear equalizers [165], or by simplified nonlinear compensators which exploit the correlations [166]. In both of these cases, the impact of the nonlinearly generated noise signal is reduced, and so predictions based on equations 24 to 29 are slightly pessimistic.

The noise generated per span varies exponentially with span length for a lumped amplifier system, but linearly with span length for an ideal lossless Raman amplified system, however, the effective length is substantially increased for the Raman system, greatly increasing the impact of nonlinearity. Since the impact of ASE on the optimum snr is greater than that of the nonlinear noise (see equation 29), it is expected that Raman amplified systems will always outperform there lumped amplified counterparts. 
Considering the impact of these two features on the optimum signal-to-noise ratio allows one to estimate that the optimum signal to noise ratio of the lossless Raman system should be higher than that of a lumped amplifier system by a factor of

$$
\frac{s n r_{\text {Raman }}}{s n r_{\text {lumped }}}=\frac{e^{\frac{2 \alpha L}{3}}}{\alpha L} \sqrt[3]{\frac{n_{s p}^{2}}{2 n_{R}^{2}}\left(\frac{\ln \left(L_{e f f} \sigma_{N L}\right)}{\ln \left(N_{a} \sigma_{N L} L\right)}\right)}
$$

where $n_{R}$ is the spontaneous emission noise factor for ideal distributed Raman [166].

For a given fiber, the performance gain obtained by switching to Raman amplification is dominated by the system bandwidth (larger bandwidths diminishing the benefit of Raman due to higher nonlinearity) and the amplifier spacing (longer spacing emphasizing the reduced noise of the Raman system). The typical achievable gains are shown in figure 15, below, for system bandwidths ranging from $50 \mathrm{GHz}$ (deep red) to $5 \mathrm{THz}$ (purple), and for amplifier spacing between 33 (dotted) and 100 (solid) km. Clearly the figure shows that the amplifier spacing dominates the difference in performance. This is because, for the ideal distributed Raman system (no variation in signal power), the performance does not vary with the amplifier spacing, however for a system with lumped amplifiers, longer spans have higher loss and degrade the optical signal to noise ratio. This gives an exponential dependence on amplifier spacing, as reflected in the exponential dependence in equation 31 (numerator of the first term). The impact of the total bandwidth on the difference in performance is very much very much lower, since this is dominated by the phase matching effects of dispersion, and is reflected in the logarithmic terms of equation 31, where the main difference is the effective lengths of the two systems. For submarine systems, where amplifiers spacing [167] and modulation format [168] are typically optimized to maximize the capacity per unit energy, it has been observed that the gain from including Raman amplification is less than $2 \mathrm{~dB}$ [169], whilst for terrestrial systems, where amplifier spacing is typically much larger, the gains from the inclusion of Raman amplified spans are much more significant.

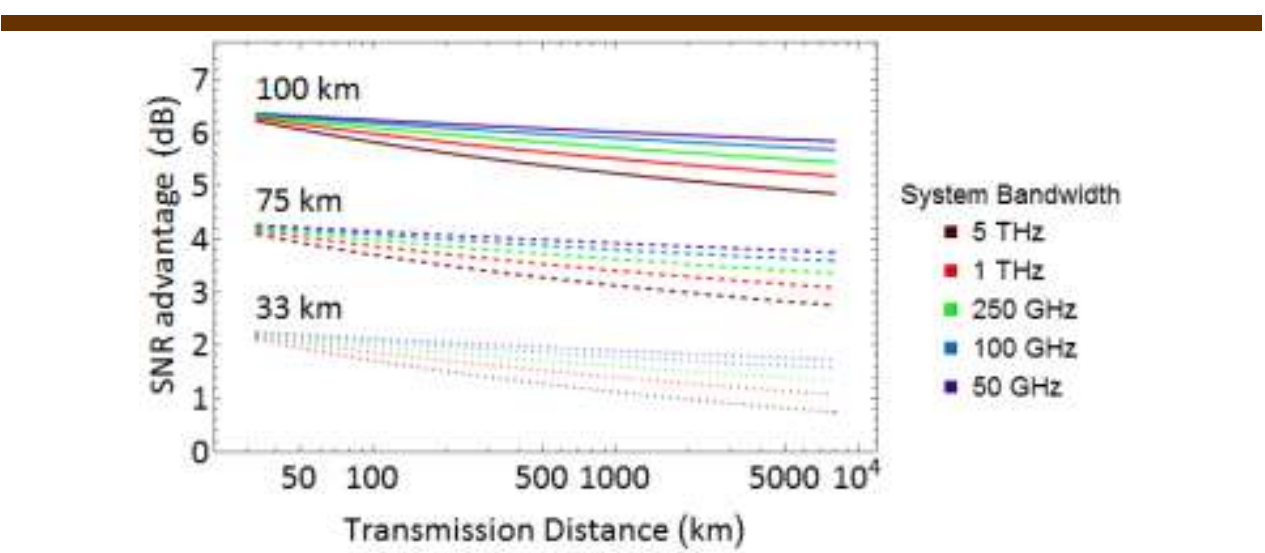

Figure 15. Theoretical performance advantage of ideal loss-less Raman amplified system compared to a lumped amplified system for amplifier spacing of 33 (dotted), 66 (dashed) and 100 (solid) km and for total system bandwidths of 50 (deep red), 100 (red), 250 (green), 1000 (blue) and 5000 (purple) GHz with amplifier noise figure of $4.77 \mathrm{~dB}$, a Raman noise coefficient of 1.17 and a loss coefficient of $0.2 \mathrm{~dB} / \mathrm{km}$.

The approach detailed above, where all noise sources are treated independently, and also independent of the signal has proved accurate for a wide range of deliberate experimental tests [170-172] and independent comparisons between predictions and experiments. However, as shown in equation 28, in certain circumstances, for example where the accumulated dispersion remains low, it is necessary to apply correction factors to account for correlations in the accumulation of nonlinear effects. Whilst this correctly identifies the magnitude of the power transferred between signals, and between signals and noise, it is in exactly these same circumstances where the resultant noise distribution deviates most strongly fromadditive white Gaussian noise both in terms of shape [173-174] and distribution [175-176]. These variations in distribution of course invalidate the additive white Gaussian noise approximations outlined above, and for systems without nonlinearity compensated may result in the nonlinear penalties being underestimated in some circumstances. Of course, the observed correlations may be exploited within the receiver signal processing [177] which would improve the performance legacy dispersion managed and dispersion shifted fiber systems upgraded with coherent transponders. Such correlations 
of course are most keenly felt within the channel bandwidth itself, and if these effects are reversed through an appropriate nonlinear impairment compensation method, such as digital back propagation (DBP), we are left with inter channel effects for more widely spaced signals, where the correlations are much lower, and the interaction between the signal and noise which is closely approximated by the additive white Gaussian noise model, especially since at least one of the interacting fields is a noise field.

\subsection{Alternative approaches to model nonlinearity in coherent transmission systems}

Table 1: Classification of channel models

\begin{tabular}{|c|c|c|c|c|c|}
\hline \multirow{2}{*}{ Memory } & \multicolumn{3}{|c|}{ Domain } & \multicolumn{3}{c|}{ Noise interactions considered } \\
\cline { 2 - 6 } Finite & Continuous & Time & {$[178-180]$} & {$[82,181]$} & S \\
\cline { 2 - 6 } & Discrete & Time & {$[143,163.182-$} & & {$[154]$} \\
\hline \multirow{3}{*}{ Infinite } & Continuous & Frequency & {$[186]$} & & {$[136,141]$} \\
& & & $140,145,156$, & & \\
& & & $162]$ & & 1151,153, \\
& Discrete & Frequency & {$[71,186]$} & & $187]$ \\
\cline { 2 - 6 } & Continuous & Time & & & {$[142,156$,} \\
& & & & & $188]$ \\
\cline { 2 - 6 } & Discrete & Time & {$[144]$} & & \\
\hline
\end{tabular}

In section 4.2 above, we have calculated the nonlinear impairments by integrating the quasi phase matched four wave mixing efficiency over the signal and noise fields injected into the fibers. This can be classified as a continuous frequency approach with infinite. Examples of classifying models in terms of memory and domain are shown in Table 1 . The derivation presented in this paper is valid for a wide range of circumstances, especially those where the signal amplitude and phase varies rapidly in frequency when compared to the four wave mixing efficiency. Such circumstances include OFDM formatted signals, highly dispersed signals and high cordiality modulation formats, where signals resemble noise $[145,163,189]$. Indeed, this approach is sufficiently successful that it has been proposed to replace such signals with spectrally shaped ASE noise in order to stress test system performance [190,191]. However, in other circumstances, alternative derivations are either more accurate, or simply offer even more tractable derivations and physical understanding. Table 1 therefore lists a selection of alternative models which the reader will find useful in these circumstances.

It is useful to note at this point that if memory is included in a formal calculation of the maximum information spectral density, then performance slightly above the optimum predicted by equations 30 and 32 may be possible using, for example, Satellite [180] or Ripple [136] constellations respectively, provided any additional assumptions are satisfied. In addition accurate channel modelling can be used as a basis for advanced methods of nonlinearity mitigation [192-194].

\section{Performance limits with nonlinearity compensation}

Section 4.2 shows that there is a clear maximum deliverable signal to noise ratio for multi-channel optical communication systems using conventional system designs. Commercially available products are rapidly approaching this limit, whilst historical trends [3,4] and industry forecasts [195-196] suggest that demand will continue to grow exponentially. Although it is perhaps unwise to rely solely on a single forecast and historical trends all other indicators confirm the need for continued growth in the volume of traffic transported across the network. The imminence of these volumes exceeding the capability of a single fiber to transport the data over the required routes hassled to widespread discussion of a capacity crunch [5]. As we saw in section 3.4, the deleterious impact of nonlinearity may be mitigated by the use of dispersion management and/or soliton transmission. In the case of soliton transmission the performance is then primarily dominated by the interaction of the signal with noise through Gordon Haus jitter [82]. However, this approach is somewhat restrictive in the use of pulse shapes, and until recently multiamplitude level solitons had not been considered [197]. In principle, the inter signal nonlinearity discussed (first term of equation 25) above is deterministic and thus can be fully compensated as was first described using a concept of inverse nonlinear transmission at the receiver [198] even in the case of solitons. Suitable compensators may be implemented digitally in the transmitter or receiver (digital back propagation (DBP)), ideally calculating the impact of nonlinearity over the full system bandwidth (and probably with a high number of steps per span) [199-202]. For a multi-channel system, it is unlikely that a single receiver would process the system, and so a nonlinear multiple-input-multiple-output signal 
processing strategy should be adopted, where each input would be the detected signal of a particular channel. Such receivers have been implemented using optical comb sources [203], and similar gain may be achieved by MIMO signal processing in a comb based transmitter [204-205]. Significant gains are possible for isolated super-channels propagating without neighbors [203-206], but for a fully populated wavelength division multiplexed (WDM) system where compensation over the full system bandwidth is not feasible, the practical limit in the ability of digital signal processing to improve the signal to noise ratio appears to be around 1-2dB [207], although optical phase conjugation (OPC) [208-209] offers the prospect of sufficiently wideband compensation. In an OPC system, the entire system bandwidth is phase conjugated after a certain length of a transmission system. If the signal is then propagated through a link with identical distortions, subject to certain symmetry conditions, linear and nonlinear effects (excluding odd ordered dispersive effects) are reversed. Following early research into the benefits of OPC for direct detection systems e.g. [210-212], which were severely constrained by nonlinearity as shown in section 3.2, the emergence of digital coherent receivers offered sufficiently superior performance to significantly postpone the need to compensate nonlinearity with 40,100 and 200Gbit/s line systems developed using predominantly linear equalizers.

\subsection{Performance limits using digital back propagation}

The nonlinear interaction between the signal components of the optical field is, in principle at least, completely deterministic, and is governed by equation 10 . If the nonlinear interaction between signals, either using DBP or OPC is substantially or completely compensated, then it is necessary to also consider the interactions between signal and noise shown in equation 25 , where the impact of an ideal nonlinearity compensator might be to cancel the inter-signal term with a length scaling factor $\zeta_{0}$. The intrinsically stochastic nature of amplified spontaneous emission (ASE) noise coupled with uncertainty over the point in the link where it is generated makes it hard to fully compensate for the nonlinear interaction between signals and ASE noise generated along the system, although it should be possible to compensate for effects involving ASE from the first amplifier. In practice, one span will have its parametric noise amplification compensated, however other links will be either under or over compensated leaving residual or inducing virtual noise amplification respectively. This is shown in figure 16, which shows the evolution of the parametric noise amplification contributions from each amplifier, assuming that the receiver is set to compensate exactly for the inter-signal nonlinearity in the receiver. All of the parametrically amplified noise from the first amplifier compensated, unfortunately, the noise from the last amplifier undergoes an effective nonlinear interaction within the nonlinear compensator, and grows. The net effect is almost no change in the total parametrically amplified noise ( $N_{a}$ is replaced by $N_{a}-1$ in equation 26$)$.

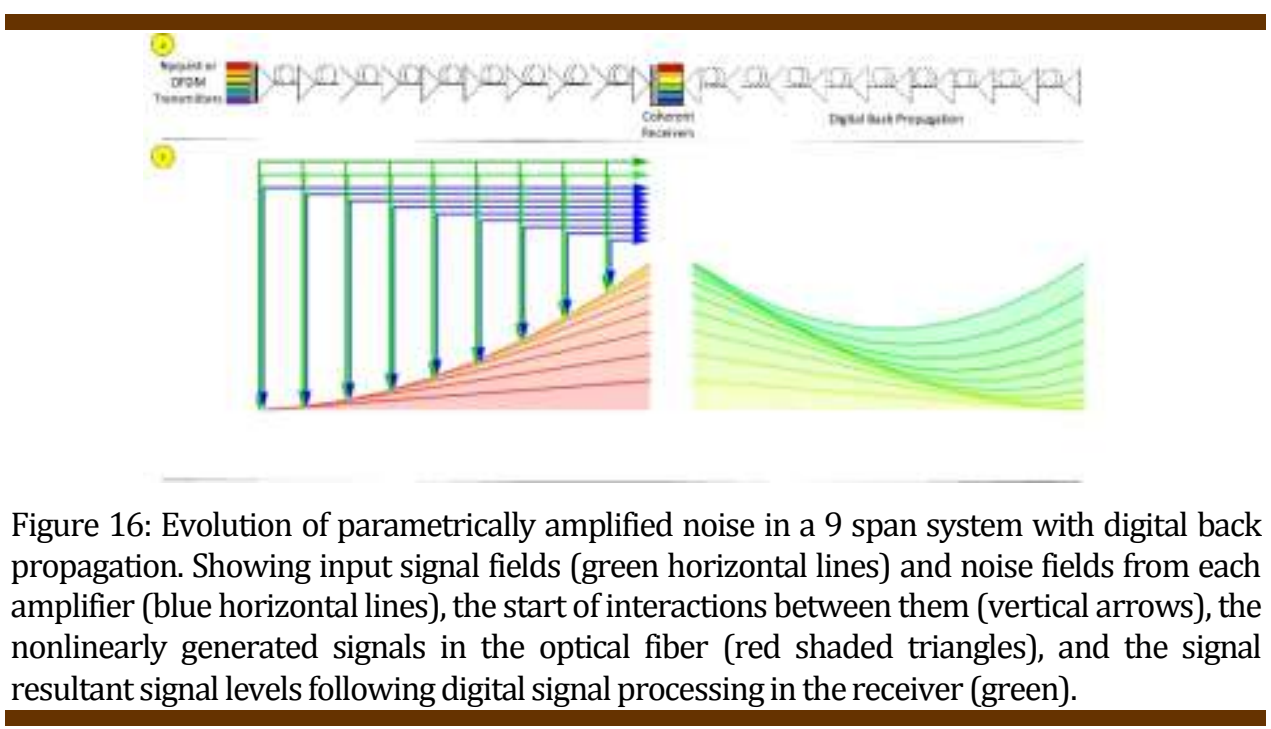

A pure ideal DBP system is thus likely to be dominated by parametric noise amplification (second term of equation 25) and assuming that $\xi_{0}=0$, and that the impact of the higher order terms is negligible, it is straightforward to show that the optimum signal to noise ratio becomes [213-214]

$$
s n r_{D B P} \cong \frac{3}{2 \sqrt{2}} \sqrt{\frac{N_{a}}{N_{a}-1}} s n r_{o p t}^{\frac{3}{2}}
$$


Or expressed in decibels, the optimum signal-to-noise ratio after DBP will be at most $50 \%$ higher (in $\mathrm{dB}$ ) than the optimum signal to noise ratio prior to compensation. The inclusion of higher order terms impacts the numerical factor. This simple expression readily indicates the maximum performance gain that may be obtained by DBP, and in particular, since the signal-to-noise ratio only increases (in $\mathrm{dB}$ ) by approximately $50 \%$, one can readily conclude that the system throughput, which depends logarithmically on the snr may also increase by at most $50 \%$. It indicates clearly that DBP has significantly higher potential impact for higher order modulation formats [215], and has served as an effective upper bound on the experimentally observed performance gains. The dashed lines in Figures 13 and 14 show the limit imposed by parametric noise amplification in close, but not exact, agreement with numerical simulations. Of course, it is not necessary to perform all nonlinearity compensation in the receiver, which in fact induces the maximum amount of virtually generated parametric noise amplification. That is, since receiver based nonlinearity compensation needs to compensate for the inter-signal nonlinearity over the full system length, it also compensates for parametric noise amplification over the full system length. For parametric noise amplification, the compensation is only strictly ideal for the noise injected by the transmitter amplifier, and the parametric noise amplification of amplified spontaneous emission noise from all in-line amplifiers is over compensated [153]. This is shown by the green shapes in figure 16, where we can see that the optimum reduction in parametrically amplified noise from receiver based digital signal processing occurs if only half of the system length is back propagated. In order to fully compensate inter signal effects, the remaining system length should be pre-compensated for in the transmitter. Indeed, analytical predictions and numerical simulations have shown that splitting the compensation 50:50 between the transmitter and receiver is optimum, further increasing the maximum possible snr by up to $1.5 \mathrm{~dB}[187,216]$. As was the case for direct detection systems, parametric noise amplification sets a hard limit on the potential performance improvement of any nonlinearity compensation system, and to extend performance beyond the limit suggested by equation 32 requires it to be taken into account in the design of the compensator.

The benefit of nonlinearity compensation is inevitably accompanied by a significant increase in the signal power of up to $\sqrt{2 s n r_{0}}$ (slightly higher with split compensation). Earlier predictions of nonlinear transmission performance (see section 3.2) were often expressed in terms of the total nonlinear phase shift experienced by the signal, with a "rule of thumb" phase shift of more than $\pi$ being a warning of a significant uncorrectable impact from nonlinearity. For a coherent transmission system with nonlinearity compensation, the optimum signal power corresponds to achieving a total nonlinear phase shift of around $\sqrt{2 N_{a} / 3}$ radians. This clearly scales with system length and may readily exceed a phase shift of $\pi$, and the nonlinear distortions should no longer be considered to be a small perturbation. Indeed, at the elevated launch powers enabled by compensation of the nonlinear effects, signal depletion and the generation of higher order mixing products, neglected in the derivation of equation 25 , will be significant during transmission. If the nonlinearity compensation has sufficient amplitude resolution, a sufficiently high sampling rate, a sufficiently short step size, and covers more than the total system bandwidth to capture any nonlinear products falling outside the signal bandwidth, then any of the additional higher order nonlinear effects which are solely dependent on the signal will also be effectively compensated. Unfortunately, as discussed above, nonlinear interactions involving the amplified spontaneous emission noise from multiple optical amplifiers may not be (fully) compensated, and it is necessary to include higher order mixing products involving this noise field. In particular, as the signal power increases parametric noise amplification products entering the second and subsequent spans may have higher power than the linear ASE noise injected at the beginning of the span. The growth of this higher order parametric noise amplification is illustrated in figure 17, where the parametrically amplified noise at the input to each span (after the $1^{\text {st }}$ span) is itself parametrically amplified by the signal. 


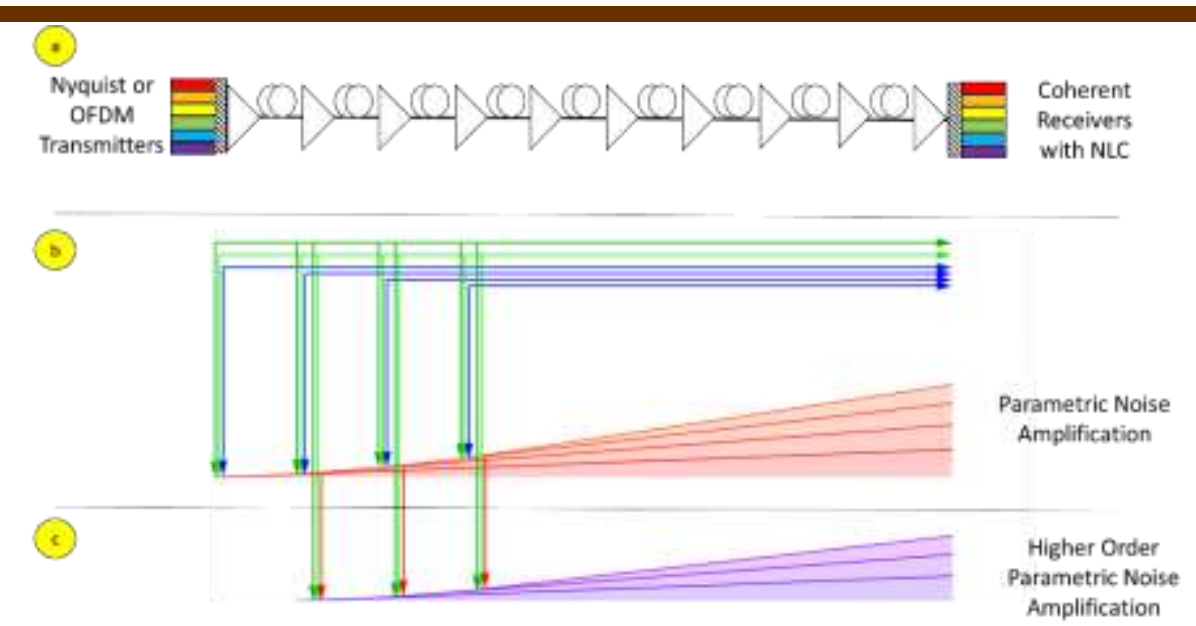

Figure 17: Evolution of $1^{\text {st }}$ order (b) and $2^{\text {nd }}$ order (c) parametrically amplified noise in a 9 span system (a). Showing input signal fields (green horizontal lines) and noise fields from each amplifier (blue horizontal lines), the start of interactions between them (vertical green and blue arrows), the $1^{\text {st }}$ order parametrically amplified noise (red shaded triangles), the onset of interaction between the signal and $1^{\text {st }}$ order parametically amplified noise (vertical green and red arrows) and the $2^{\text {nd }}$ order parametrically amplified noise. Only interactions originating in the first few spans are shown for simplicity. Adapted from [152].

To account for this additional noise term, an additional term $D_{H}$ is added to equation 25 representing the higher order parametric noise amplification for both lumped (contributions from each noise source added) and distributed (contributions integrated over the fiber length) systems [152, 213]:

$$
D_{H}=\zeta_{1 H} D_{S}^{2} D_{a}
$$

where

$$
\zeta_{1 H}=\left\{\begin{array}{c}
\frac{1}{6}\left(N_{a}+1\right) N_{a}\left(N_{a}-1\right) \Gamma^{2} D_{S}^{2}, \alpha>0 \\
\left(\delta N_{a} L D_{s}\right)^{2} \ln \left(\sigma_{N L} N_{a} L e^{-5 / 6}\right), \alpha=0
\end{array}\right.
$$

In the remainder of this section we will assume that signal-signal interactions are fully compensated to formulate the limits due to signal-noise interaction effects for a system without memory. Setting $\zeta_{0}=0$ in equations (25) and (34) allows the optimum signal-to-noise ratio to be calculated taking into account nonlinear noise, nonlinear phase noise, parametrically amplified noise and higher order parametric noise amplification.

Figures 18 and 19 directly illustrates the importance of considering the second order parametric noise amplification in nonlinearity compensated systems. Fig. 18 shows the variation in nonlinear noise for a lumped system with/without digital back propagation receiver observed using numerical simulation of equation 10 using the split step Fourier method (VPITransmissionMaker 9.5), whilst Fig. 19 shows the noise evolution in ideal Raman system that uses DBP or no nonlinearity compensation. In both cases employing DBP the noise observed in the numerical simulations is under-estimated by analytical theory which neglects second order contributions. By neglecting nonlinear phase noise and nonlinear noise, it can readily be shown that the omission of higher order parametric noise amplification leads to an over estimate of $0.7 \mathrm{~dB}$ in the optimum signal to noise ratio. 


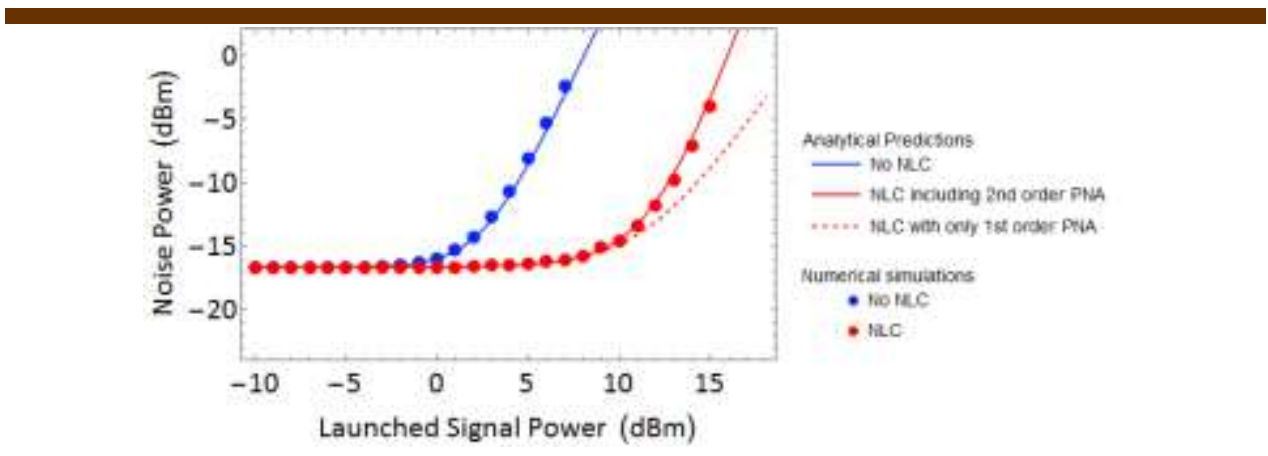

Figure 18: Single channel 28Gbaud PM-QPSK noise evolution in zero PMD fiber systems for a 12 span system using lumped amplifiers with a noise figure of $6 \mathrm{~dB}$ and $100 \mathrm{~km}$ spans of standard single mode fiber. Showing complete theory considering the $1^{\text {st }}$ order and $2^{\text {nd }}$-order signal noise products (solid lines), approximate theory considering only the $1^{\text {st-order signal- }}$ noise product (dashed line), and simulation results (dots). Data is shown for a system where the electronic signal processing only compensates for linear impairments (Blue) and where it also compensates for nonlinear effects (red) and data is replotted from [152].

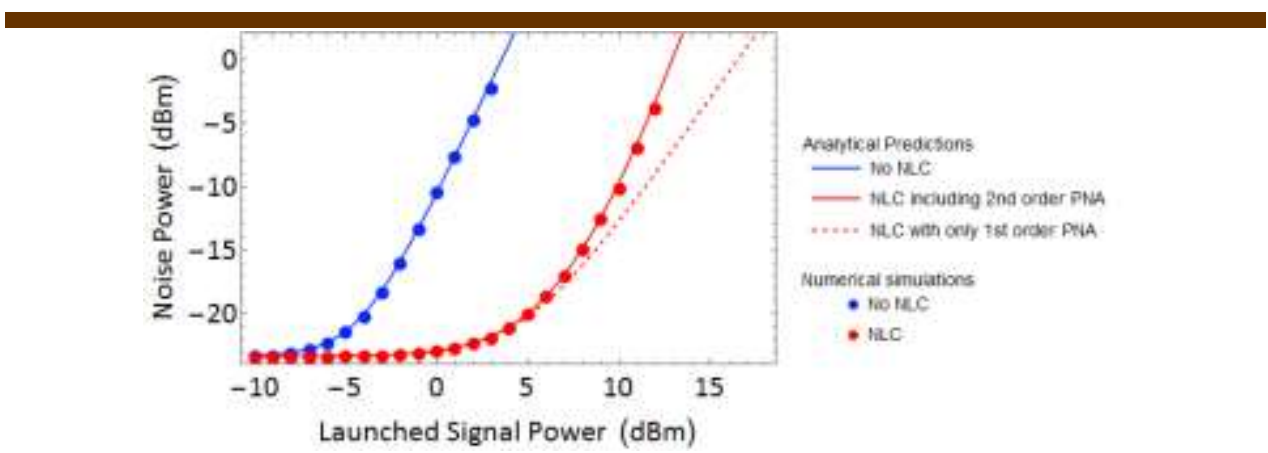

Figure 19: Single channel 28Gbaud PM-QPSK noise evolution in zero PMD fiber systems for a 12 span system assume ideal Raman amplification with total noise power spectral density of $11 \times 10^{-17} \mathrm{~W} / \mathrm{Hz}$ inserted every $1 \mathrm{~km}$ in the link, and spans comprising $100 \mathrm{~km}$ of standard single mode fiber. Showing complete theory considering the $1^{\text {st }}$ order and $2^{\text {nd }}-$ order signal noise products (solid lines), approximate theory considering only the $1^{\text {st }}$-order signal-noise product (dashed line), and simulation results (dots). Data is shown for a system where the electronic signal processing only compensates for linear impairments (Blue) and where it also compensates for nonlinear effects (red) and data is replotted from [152].

We can also see from Fig 19 that the nonlinear noise generated by the Raman system is higher than that of the discrete system, but that consideration of higher order parametric noise amplification still provides a good fit to the numerical simulations. Despite the addition of a term scaling quartically with the signal power in equation 25 , the optimum signal-to-noise ratio may still be readily estimated from the maximum value of equation 24 in the following two cases; (1) without nonlinearity compensation where we assume inter-signal nonlinearity to be dominant ( $D_{N L}=N_{\mathrm{a}} \Gamma D_{S}^{3}$ for a lumped amplifier system), and (2) where we assume ideal nonlinearity compensation including the first two orders of parametric noise amplification $\left(D_{N L}=\left(3 N_{\mathrm{a}}\left(N_{\mathrm{a}}+1\right) / 2+\left(N_{\mathrm{a}}+1\right) N_{\mathrm{a}}\left(N_{\mathrm{a}}-1\right) \Gamma D_{S}^{2} / 6\right) \Gamma D_{S}^{2} D_{a}\right.$ for a lumped amplifier system) The improvement in signal-to-noise ratio is given by [213];

$$
s n r_{D B P}=0.9 s n r_{o p t}^{\frac{3}{2}}
$$

Comparing equations 32 and 35, we observe that they are different only in terms of the scaling factor, with equation 32 over estimating the optimum signal to noise ratio by at least $0.7 \mathrm{~dB}$, and is only dependent the 
number of amplifiers in the link. This achievable performance gain is illustrated by the dotted lines in figures 13 and 14, and the theoretical predictions show excellent agreement with the numerical simulations.

We have detailed above the maximum possible benefit from compensating the nonlinearity at the ends of a transmission link. This assumes potentially impractical ultra-wide bandwidth digital signal processing. Figure 20 illustrates a selection of reported investigations into nonlinearity compensation, including numerical simulations (open symbols) and experimental demonstrations (closed symbols) using a wide variety of compensation techniques. Note, as discussed in section 2 , that $Q$ factors are often reported by converting the BER to signal-to-noise ratio assuming QPSK modulation, irrespective of the actual modulation format used. Here we report the optimum performance levels converted to electrical signal to noise ratio. Digital nonlinearity compensation techniques developed to date include direct digital back propagation, essentially solving equation 10 in each receiver [217-223], various simplified forms of back propagation [224-225], various coding schemes where duplicate information is transmitted (see section 5.3), Volterra series estimation [226], pilot tone estimation [227-228] and look-up tables [229]. For details of how to implement such schemes, the reader is directed to these sources, and to recent reviews of electronic nonlinearity compensation $[202,230]$ and references therein. The numerical values of Figure 20 and the associated references are summarized in Table 2.

Table 2: Selected experimental demonstrations of digital nonlinearity compensation in high capacity transmission systems

\begin{tabular}{|c|c|c|c|c|c|}
\hline & & & & \\
First Author & Ref & Rate $\mathbf{T b} / \mathbf{s})$ & Length $\mathbf{( k m )}$ & $\Delta \mathbf{P}(\mathbf{d B})$ & $\Delta \mathbf{Q}(\mathbf{d B})$ \\
\hline Tanimura & {$[217]$} & .2 & 2160 & 4 & 1 \\
\hline Mussolin & {$[218]$} & .2 & 250 & 4 & 1 \\
\hline Sackey & {$[219]$} & .2 & 800 & 3 & 2.2 \\
\hline Silva & {$[220]$} & .25 & 1,700 & & 1.5 \\
\hline Zhang & {$[221]$} & .3 & 2560 & 2 & .8 \\
\hline Maher & {$[222]$} & .3 & 1940 & 3 & 1.4 \\
\hline Omiya & {$[223]$} & .4 & 720 & 0 & 1 \\
\hline Temprana & {$[205]$} & .5 & 2890 & 4 & 2.4 \\
\hline Xia & {$[224]$} & .8 & 2560 & 2 & 2.2 \\
\hline Maher & {$[231]$} & .8 & 1280 & 4 & 1.6 \\
\hline Yankov & {$[232]$} & .8 & 1,400 & 1 & .35 \\
\hline Fontaine & {$[203]$} & 1.2 & 960 & 2 & 1 \\
\hline
\end{tabular}

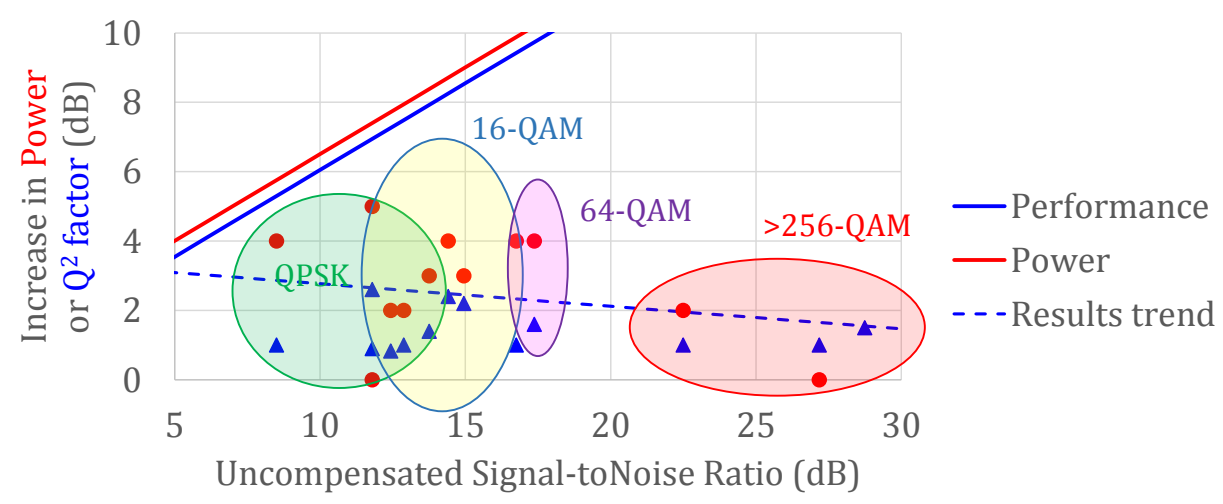

Figure 20: Comparison of experimental coherent transmission systems using electronic nonlinearity compensation (symbols) against theoretical maximum improvements (lines), showing increase on optimum launch power (red) and $\mathrm{Q}^{2}$-factor (blue). Dashed blue line shows the experimentally observed trend in performance gain, reducing with increasing modulation order.

Numerical simulations where the full signal bandwidth is processed digitally, reported by a wide variety of groups, show excellent agreement with the analytical predictions presented in this paper. However, 
where only part of the simulated signal bandwidth is used for the backpropagation, the performance is significantly reduced, with typical signal-to-noise ratio gains using standard single mode fiber in the region of 1.5 to $2 \mathrm{~dB}$. The reported performance gains are significantly lower than the theoretical predictions, and reduce as the modulation format increases, suggesting that uncompensated nonlinearity has a greater impact on higher order modulation formats. Experimentally restrictions in signal processing bandwidth, vertical resolution, processing complexity and the impact of polarization mode dispersion all variously combine to reduce the benefit of digital back propagation to a few $\mathrm{dB}$.

\subsection{Performance limits using optical phase conjugation}

The use of phase conjugation to reverse linear [208] and nonlinear [209] distortions is well known, and has many applications outside of telecommunications. Within the communications field, subject to certain constraints, optical phase conjugation (OPC) provides compensation of deterministic linear and nonlinear impairments. Furthermore, as we shall see later, provides some relief against the impact of stochastic impairments [187]. The overall complexity of an OPC based system is greatly reduced through the use shared optical resources, since only a single pair [233] of OPC devices may process the entire WDM signal. Complexity is further reduced by reducing the signal processing load of digital coherent receivers associated with, for example, chromatic dispersion compensation, enabling simple mixed signal designs originally developed for high capacity short reach systems [234] to be considered for long haul transmission.

A system employing OPC offers full modulation format transparency and is thus fully backwards compatible and future proof. However careful optimization of the design is required to ensure sufficient link symmetry in terms of ensuring that as much of the signal power and dispersion evolution in each segment of the transmission link is matched to that in the compensating segment. Raman amplification, which was shown above to offer superior net performance for a very wide range of transmission systems, is one promising suggestion to provide high levels of symmetry. It has been proposed that a useful quantification of the symmetry for a system with uniform dispersion may be found by normalizing the integral of power difference between the forward propagating signal in the one segment, and a backwards propagating signal in the compensating signal to the integral of the signal power in either segment [235, 236]. This may be generalized to allow for a lumped dispersive element associated with the OPC device itself to give a figure of merit of

$$
\eta_{S}=\frac{\int_{0}^{L^{\prime}}\left|p(z)-p\left(L^{\prime}-z\right)\right| d z+\int_{L^{\prime}}^{N_{a} L / 2}\left|p(z)-p\left(N_{a} L / 2+L^{\prime}-z\right)\right| d z}{2 \int_{L^{\prime}}^{N_{a} L / 2}|p(z)| d z}
$$

Where $p(z)$ represents the evolution of the signal power throughout the system $L$ the length of each span and $L$ ' the equivalent length of a dispersive element located at the OPC site. The equivalent length is the length of transmission fiber compensated for by the lumped dispersive element, which could be a dispersion compensating fiber, a chirped fiber grating or an all pass filter.

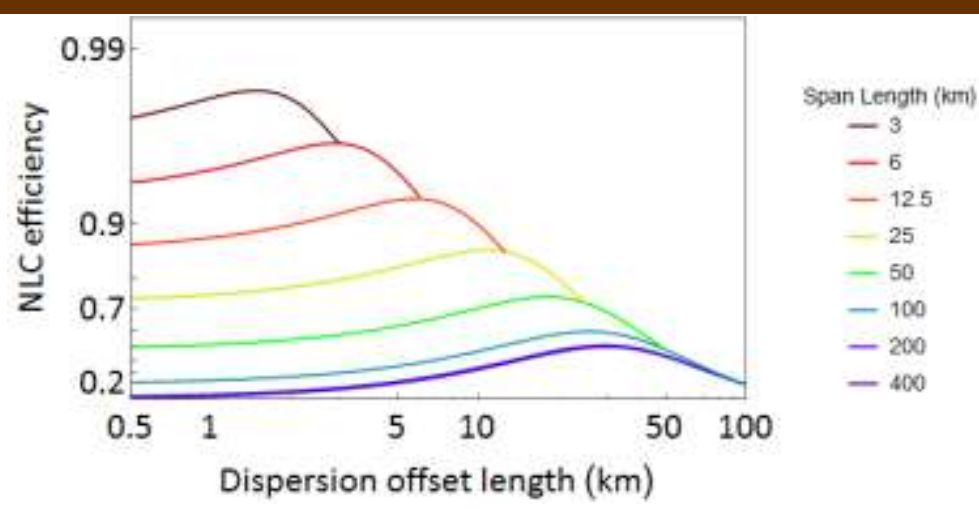

Figure 21. (a) EDFA based system with (blue to dark red) 400, 100, 50, 25, 12.5, 6, 3 km span lengths as a function of the equivalent length of additional dispersion compensation added at the OPC site. Black line is locus of the peaks (readily calculated analytically). NLC - nonlinear compensation.

Whilst the basic form of equation (36), with $L^{\prime}=0$, was originally proposed for the ideal case of a laboratory environment, with identical spans, such that the integrals need to only be performed over a single span, 
the figure of merit may be readily calculated for any configuration, including unequal span lengths, launch powers and even additional spans on one side of the OPC. It has been assumed [237] that the figure of merit is directly equivalent to a reduction in compensation efficiency, such that the compensation efficiency is simply $1-\eta_{S}$ and thus $\zeta_{0}=N_{a} \eta_{S}$ in equation 25 .

Figures 21 and 22 illustrate the predicted influence on dispersion power symmetry on the maximum achievable nonlinear compensation efficiency for a mid-span OPC system for lumped (figure 21), backwards pumped Raman and bidirectionally pumped Raman amplified systems (figure 22). The closer the NLC efficiency is to unity, the more complete is the compensation of the inter signal nonlinearities. For conventionally deployed lumped amplification systems (span lengths greater than $50 \mathrm{~km}$ ), the compensation efficiency is less than $50 \%$ without a lumped dispersive element collocated with the OPC $\left(L^{\prime}=0\right)$. This efficiency reduction arises because the accumulated dispersion where the signal powers are highest are effectively offset by one span length of fiber (adjusted for the nonlinear effective length). By including a purely linear dispersion compensating element in line with the OPC, this mismatch between the values of accumulated dispersion as a function of signal power is reduced and the nonlinear compensation efficiency greatly increase, with the impact being substantial where the span length exceeds the nonlinear effective length.

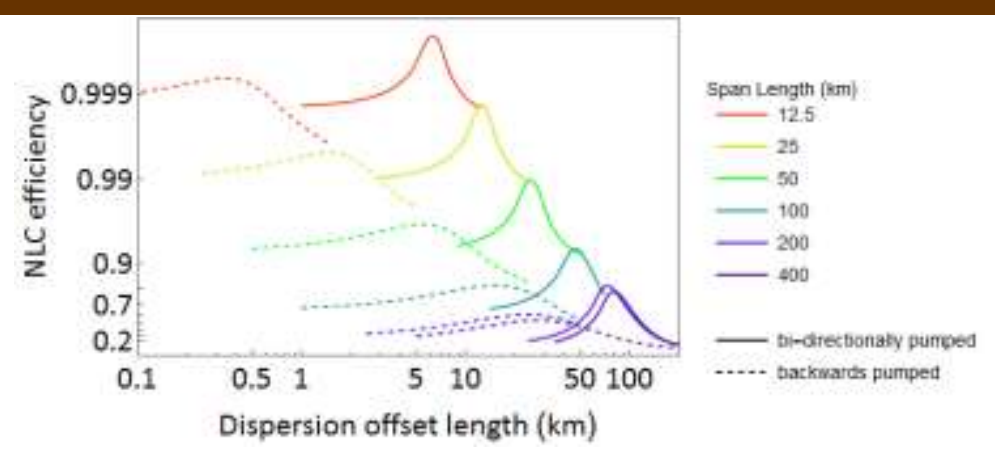

Figure 22. (b) Maximum nonlinear compensation efficiency of a quasi-Lossless Raman amplified OPC system as a function of the equivalent length of additional dispersion compensation added at the OPC site with (dark blue to light green) 400, 200, 100, 50, 25 and $12.5 \mathrm{~km}$ span lengths. Dashed lines, backwards (first order) pumped, solid lines bi-directional (equal pump power, first order). NLC - nonlinear compensation.

For a Raman amplified system, the signal gain at the fiber output restores the signal power to its maximum value at the same accumulated dispersion. This immediately increases the compensation efficiency from $20 \%$ to over $60 \%$ for a $100 \mathrm{~km}$ span length for both backwards (dashed) and bi-directionally (solid) pumped (first order) systems. Without a dispersive element, the backwards only system consistently gives higher compensation efficiencies than bi-directional pumping since the latter actually increases the effective length of the signal at the input to the fiber, somewhat enhancing the original problem. For both pumping schemes, the addition of an appropriate dispersion compensating element enhanced the performance. The gains are more significant for the bi-directionally pumped system, whilst for the backwards pumped system, it is possible to actually degrade the compensation efficiency by selecting the incorrect value of dispersion. A fully optimized first order bi-directionally pumped Raman amplified system, with $200 \mathrm{~km}$ spacing between Raman pumps would provide greater compensation efficiency than a $25 \mathrm{~km}$ spaced lumped amplification system. Such comparisons strongly suggest that OPC systems should ideally be accompanied by Raman amplification. Indeed, as Raman systems without nonlinearity compensation are typically expected to outperform their lumped counterparts it makes sense to first deploy Raman amplification before considering OPC. Even greater power symmetry is possible if optimized second order Raman pumping is employed [238-239], allowing the prospect of near complete compensation of nonlinear impairments to be considered. 


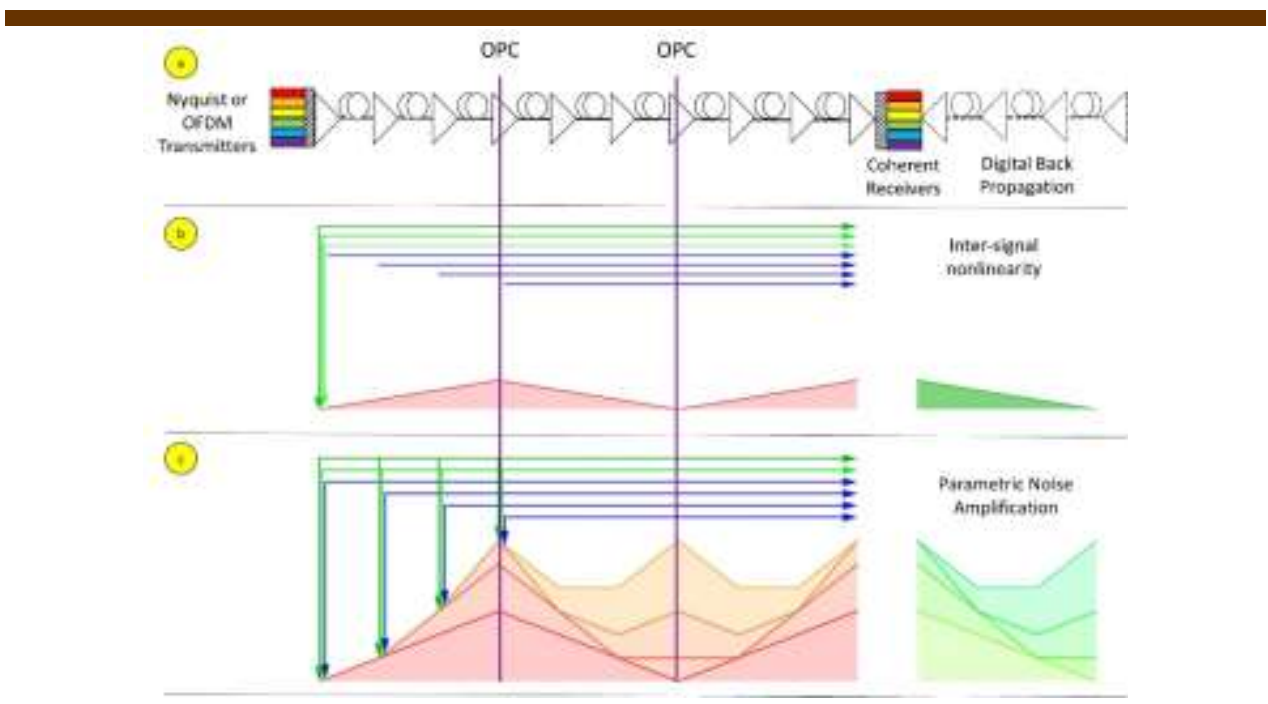

Figure 23: Interacting fields contributing to total nonlinear noise power in a two OPC, nine span system supplemented by digital back propagation, showing input signal fields (green horizontal lines) and noise fields from each amplifier (blue horizontal lines), the start of interactions between them (vertical arrows) and the nonlinearly generated signals (red, orange mustard and yellow shaded triangles). OPCOPC locations identified by vertical purple lines. Each row shows a different interaction, specifically; inter signal interaction (b), parametrically amplified noise (c). Nonlinear phase noise and nonlinear noise is not shown for simplicity. For (c) the total noise is the sum of contributions from all amplifiers and is represented by the maximum height of the shaded region. The figure also shows impact of digital nonlinearity compensation to either compensated or enhance nonlinear products as green triangles. Adapted from [187].

Whilst the concept of complete cancellation of inter signal nonlinearity is straightforward using a mid-link OPC, evolution of parametric noise amplification becomes more complex in a system with one or more OPCs. The generic concepts for inter-signal and parametric noise amplification are illustrated in figure 23. In this example, signals interact from the transmitter and the nonlinear noise grows. After the first OPC this nonlinear noise growth is reversed. Since there is no nonlinear noise after the sixth span (for this configuration), the second OPC has no impact on the nonlinear noise, and in the final segment of three spans, the nonlinear noise grows again. In this example, after coherent detection, digital back propagation is used to compensate the nonlinear effects of these final three spans. The same evolution occurs for the parametric amplification of noise injected by the transmitter amplifier, and this contribution to the total nonlinear noise is also compensated. After a parametrically amplified noise contribution passes through an $\mathrm{OPC}$, the parametric amplification process is reversed, and the net noise gain is reduced. Of course, if there are additional spans between the OPC and the receiver the system is overcompensated, and the parametric noise amplification grows again [187, 240]. With multiple in-line amplifiers, as with all parametric noise amplification processes, it is impossible to determine from which amplifier a particular noise contribution originated from, and some amplifiers will have their parametric noise amplification fully compensated, but others will be wither under or even over compensated. If multiple OPCs are employed (or a combination of OPCs and DSP based nonlinearity compensators) then the growth of parametric noise amplification is limited to the growth experienced between two OPCs (and/or transponders). For the 9 span system shown in figure 23, the use of two OPCs reduces the parametric noise amplification considerably, limiting the maximum parametric noise amplification to that which would be experienced in an isolated 3 span segment and by $4.77 \mathrm{~dB}$ overall, whilst adding split digital compensation further reduces the impact of parametric noise amplification by $2.7 \mathrm{~dB}$. The location of OPCs should of course be optimized to minimize the parametric noise amplification, subject to the constraint that the inter-signal nonlinearity is fully compensated. Previous work looking directly at parametric noise amplification suggested that equally spaced OPCs would give the best performance, splitting any necessary DSP between transmitter and receiver to minimize the residual noise [187]. Earlier work into other manifestations of the nonlinear interaction between signal and noise [88] have suggested that the length of the first and last spans should be varied, especially if receiver signal processing only is taken into 
account, where it was found that the optimum placement for a single OPC was at $2 / 3^{\text {rd }}$ of the total link length, whilst more recent work suggests that the first and last segments in a link should contain half the number of spans as any other segment [241].

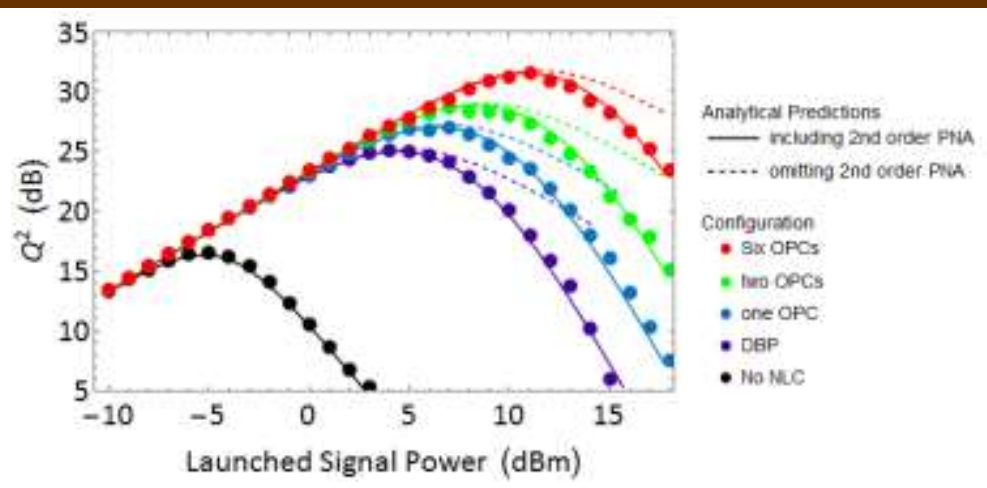

Fig. 24: Performance of an 8 channel 28Gbaud PM-QPSK systems over a 12x100km ideal Raman amplified link with total noise PSD of $11 \times 10-17 \mathrm{~W} / \mathrm{Hz}$ inserted every $1 \mathrm{~km}$. (Solid line) theory considering the 1st-order and 2nd-order signal noise products, (dashed line) theory considering only the 1st-order signal-noise product, (dots) simulation results. Black, without nonlinearity compensation; purple, receiver based DBP; blue, one OPC; green two OPCs; and red, six OPCs. No digital back propagation employed for the OPC cases. Adapted from [152].

Considering the simplest configuration, placing a single OPC in the middle of the transmission path leads to a signal to noise ratio gain of up to $1.27 \mathrm{snro}^{(3 / 2)}$, equivalent to a $1.17 \mathrm{snro}^{(1 / 3)}$ reach enhancement [187, 213]. Reach enhancements exceeding a factor of 2 are clearly possible for higher order modulation formats (requiring a large baseline snr), suggesting that nonlinearity compensation may outperform using an opto-electronic regenerator. Indeed using highly accurate wideband transmitter side nonlinearity compensation and optical frequency combs reach doubling has been achieved for 16QAM ( $s r_{0}=10.5 \mathrm{~d} B$ theoretically allowing $160 \%$ reach enhancement) and reach tripling has been achieved for $64 \mathrm{QAM}$ ( $s n r_{0}=14.7 \mathrm{~dB}$ theoretically allowing $260 \%$ reach enhancement). These results are clearly in line with the predictions of the nonlinear Shannon limit imposed by parametric noise amplification. Calculation of the limit when using multiple in-line OPCs in an ideal Raman amplified system is a straightforward matter of summing the net parametric noise amplification $[187,216]$, resulting in length scaling factors for first and second order parametric noise amplification of [213]

$$
\begin{gathered}
\zeta_{1}=\frac{N_{a}^{2}}{N_{O P C}+1} \frac{\delta L}{\Gamma} \ln \left(\sigma_{N L} \frac{N_{a}}{N_{O P C}+1} L e^{-1 / 2}\right) \\
\zeta_{1 H}=\frac{\left(\delta N_{a} L D_{S}\right)^{2}}{N_{O P C}+1} \ln \left(\sigma_{N L} \frac{N_{a}}{N_{O P C}+1} L e^{-5 / 6}\right)
\end{gathered}
$$

Where $N_{O P C}$ represents the number of uniformly spaced OPCs. This results in a further improvement in the optimum signal to noise ratio of $\sqrt{1+N_{O P C}}$, or a further increase in reach of $\sqrt[3]{1+N_{O P C}}$. Note that due to the elevated launch powers at the optimum signal to noise ratio (which can be readily shown to be $P_{O P C}=P_{o p t} \sqrt{1+N_{O P C}} \sqrt{s n r_{0}}$ ), it is necessary to consider the higher order parametric noise amplification products. The resultant performance benefits are shown in Figure 24, where numerical simulations and the predictions of equations 25 and 37 are compared for the case of ideal Raman amplified transmission systems.

OPC combined with distributed Raman amplification clearly promises significant performance enhancement over systems dominated by inter signal nonlinearities. Whilst there are concerns that each individual device will suffer a high power consumption (often in the region of 1W [237]) this power consumption should be offset against the simultaneous impairment compensation across all WDM channels simultaneously. Considering only the compensation of chromatic dispersion, a beneficial side effect of the nonlinearity compensation offered by OPC, it would be possible to design coherent receivers with greatly simplified equalizer structures. Since chromatic dispersion compensation typically accounts for some $30 \%$ of the integrated circuit consumption, and current merchant DSP chips based on $22 \mathrm{~nm}$ 
CMOS technology have typical power consumptions of around 10W [242], for a half loaded WDM system (around 5033 Gbaud channels) the net transponder energy savings is some 150W per end, readily allowing for the inclusion of a number of OPC devices within the transmission link.

The combination of a net reduction in power consumption with improved signal to noise ratio suggests that there is a compelling case for the use of OPCs. Unfortunately, experimental results fall somewhat below the ideal case. For example, nonlinearity compensation of a total bit rate $2.4 \mathrm{Tbit} / \mathrm{s}$ using a single dual-band OPC allowed a 50\% increase in reach for 6 simultaneously transmitted 400Gbit/s 16QAM super-channels with an 18\% power asymmetry (75 km link length) over standard single mode fiber [158]. Transmission over dispersion shifted and flattened fiber, using Raman amplification and a single OPC enabled a significant 3dB increase in the margin of a $2000 \mathrm{~km} 4 \times 67.25 \mathrm{Gbaud}-16 \mathrm{QAM}$ WDM system, but still less than the expected performance [243]. Figure 15 summarises coherently detected transmission experiments employing OPCs, and compares the reported system improvements with the theoretical predictions. The best observed results confirm the general trends predicted analytically, vis, increase numbers of OPCs increases the performance gain, and the potential performance gain increases with the order of the modulation format. Both of these are critical features of nonlinearity compensation schemes. The monotonic enhancement with the number of devices ensures that reasonable performance gains (7 reports exceeding $2 \mathrm{~dB}$ performance gain) may be readily achieved. Similarly, increased performance are especially welcome for higher order modualtion formats, which are highly susceptible to impairements of any kind, and which would be enabled by nonlinearity compensation in the first place [160]. However, there is a clear offset from the theoretical performance limits (solid lines in figure 25), and the signal-to-noise ratio performance is often more than $3.5 \mathrm{~dB}$ away from the theoretical predictions (dashed line). There are various reasons for this, including OSNR degradation for the inclusion of the OPC [eg 244], symmetry effects as descibed above, finite bandwidth and polarisation mode dispersion [245]. Numerical quantities and references for Figure 15 may be found in Table 3.

Table 3: Experimental demonstrations of coherent transmission aided by Optical Phase Conjugation

\begin{tabular}{|c|c|c|c|c|c|c|}
\hline First Author & Ref & $\begin{array}{c}\text { No } \\
\text { PC }\end{array}$ & $\begin{array}{c}\text { Rate } \\
\mathbf{( T b / s )}\end{array}$ & $\begin{array}{c}\text { Length } \\
\mathbf{( k m )}\end{array}$ & $\begin{array}{c}\Delta \mathbf{P}(\mathbf{d B}) \\
\text { Expt } \\
\text { (theory). }\end{array}$ & $\begin{array}{c}\Delta \mathbf{Q} \text { (dB) } \\
\text { Expt } \\
\text { (theory) }\end{array}$ \\
\hline Jansen\$ & {$[246]$} & 1 & 0.88 & 7,100 & $(6.5)$ & $1.5(6.0)$ \\
\hline Pelusi & {$[247]$} & 1 & 0.12 & 191 & $1.8\left(5.2^{*}\right)$ & $2.7\left(4.7^{*}\right)$ \\
\hline Morshed & {$[248]$} & 1 & 0.2 & 800 & $4.5(11)$ & $2(10.5)$ \\
\hline Pelusi & {$[249]$} & 1 & 1.2 & 800 & $4(7.7)$ & $2(7.2)$ \\
\hline Solis-Trapala & {$[250]$} & 12 & 0.24 & 728 & $3\left(4.0^{*}\right)$ & $1\left(3.5^{*}\right)$ \\
\hline Phillips & {$[237]$} & 1 & 0.7 & 10,000 & $5(5.5)$ & $1.5(5)$ \\
\hline Hu & {$[244]$} & 10 & 0.8 & 6,000 & $5\left(10.8^{*}\right)$ & $1\left(9.2^{*}\right)$ \\
\hline Vokovic & {$[251]$} & 1 & 0.15 & 800 & $3(9.7)$ & $2(9.3)$ \\
\hline Sackey & {$[219]$} & 1 & 0.5 & 800 & $1(8.4)$ & $.9(7.9)$ \\
\hline Yoshida & {$[252]$} & 1 & 0.24 & 400 & $2(10.1)$ & $.5(9.7)$ \\
\hline Solis-Trapala & {$[241]$} & 1 & 0.144 & 693 & $4(10.6)$ & $5(10.1)$ \\
\hline Ellis & {$[158]$} & 1 & 4 & 2,000 & $3(6.4)$ & $1.9(6)$ \\
\hline Namiki & {$[253]$} & 12 & 1.1 & 144 & & 2 \\
\hline Umeki & {$[254]$} & 12 & 13.6 & 3,840 & 4 & 0.5 \\
\hline
\end{tabular}

\$ Differential detection, ${ }^{*}$ Theoretical limit adjusted for number of in-line OPCs 


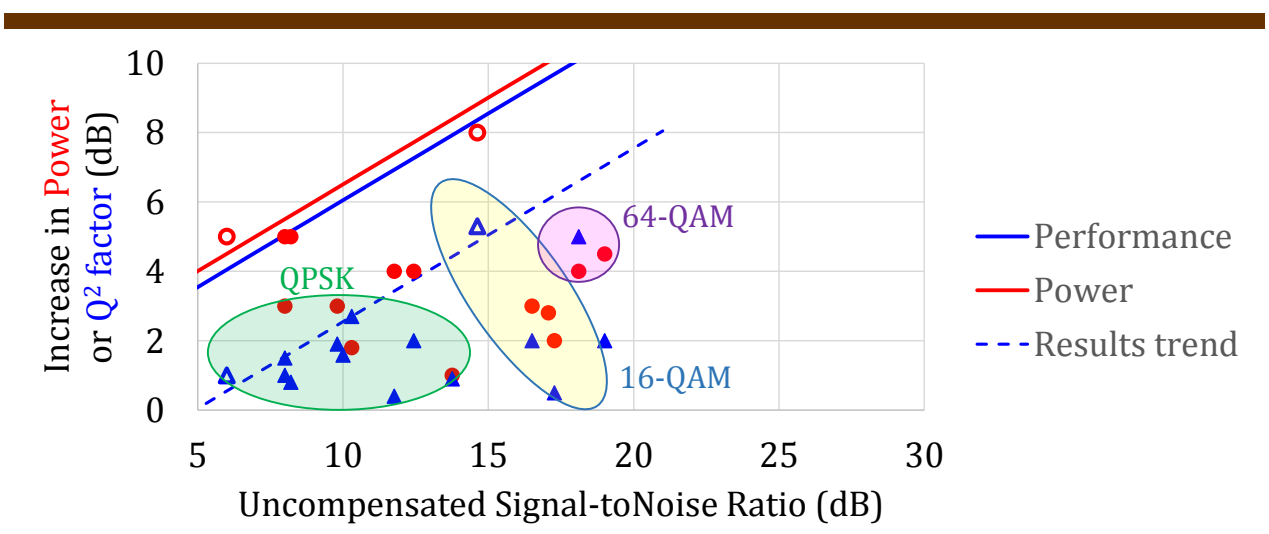

Figure 25: Comparison of experimental coherent transmission systems using optical phase conjugation (symbols) against theoretical maximum improvements (lines), showing increase in optimum launch power (red) and $\mathrm{Q}^{2}$-factor (blue) for systems with one (filled) or many (open) OPCs. Dashed blue line is a guide to the eye for the achieved gains in $\mathrm{Q}^{2}$ and indicates that performance is approximately $4 \mathrm{~dB}$ lower than the theoretical prediction.

\subsection{Performance limits using parallel data transmission}

Simulations have shown DBP to be a useful technique resulting in transmission performance limited by interactions between signal and noise [216] or by polarization mode dispersion [151]. However, its implementation is complex, multiplying the digital equalizer complexity by several factors, even when simplified structures are employed [225]. This complexity increases rapidly if compensation over multiple wavelength channels is performed. It has been recently proposed to polarization multiplex two signals combined with each other's phase conjugated copies over the same transmission line [255] for the purpose of tolerance to polarization dependent loss. This so called Polarization Time Coding was shown through numerical simulations to be resistant to the nonlinear effect of polarization scattering. Recently this concept has been generalized and experimentally demonstrated using a single data channel and its conjugate copy. The copy may be multiplexed in any available dimension, including polarization [256257], wavelength channel [258], time [259] and subcarrier frequency [227]. Ideally the signal and its conjugate copy would experience identical (or deterministically scaled) nonlinear impairments, and would accumulate statistically independent ASE noise. At the receiver, the two signals are conjugated a linearly combined to recover the original signal(s). In the case of Phase Conjugate Twin Waves, since ASE noise is uncorrelated but the two copies of the signal are correlated the signal to noise ratio is increased by $3 \mathrm{~dB}$ (this principle applies to an arbitrary number of copies). The anti-correlated nonlinear effects add destructively and the deterministic nonlinear impairments are in principle fully cancelled. Ideally, this results in an improved signal to noise ratio of $1.8 \operatorname{snr}^{(3 / 2)}$, where snrois the signal to noise ratio of one uncompensated copy, or an improvement of $2.5 \mathrm{~dB}$ plus $50 \%$ of the original $s n r$ in $\mathrm{dB}$ [161], enabling significant increases in reach. Note that $3 \mathrm{~dB}$ of this improvement arises from sending an additional copy. This additional copy clearly also occupies the same amount of bandwidth as the original signal, and so the combination of signal and copy occupy twice the bandwidth as the signal alone. Consequently, despite the attractively simple signal processing (a few additions and phase inversions) little net enhancement in total system throughput is achieved from this approach.

The range of systems where transmission of an additional conjugate copy enhances performance may clearly be improved by reducing the additional bandwidth required. This may be achieved by only transmitting one conjugate for every $\mathrm{n}^{\text {th }}$ data signal and, provided the nonlinear impairments are sufficiently identical as is the case for adjacent channels in an OFDM system, estimate the nonlinear impairment on other channels [227]. Clearly the nonlinear mitigation is somewhat less than conjugating every signal, but due to the reduced excess bandwidth net performance gains in the region of $1.5 \mathrm{~dB}$ have been observed. A more straightforward approach is the multiplexed conjugate coding of pairs of signals [260] fully generalizing the $2 \times 2$ MIMO approach of [256], and has recently been called phase conjugate coding. This approach maintains the full nonlinearity mitigation benefit, but losses the signal to noise ratio benefit of coherent superposition available when only one signal and its conjugate are used. In this case the maximum potential signal-to-noise ratio gain is simply 0.9.snro(3/2), and benefits are observed for all uncompensated signal-to-noise ratios. Clearly, the benefits of both of these techniques are not restricted 
to the transmission of a single additional copy. In the general case of multiple copies $\left(N_{C}\right)$ and assuming ideal compensation of correlated inter-signal nonlinear effects and inserting the optimum signal-to-noise ratio into the Shannon-Hartley theorem gives the maximum rate at which information could be transmitted as

$$
\frac{C}{B}=\frac{2 N_{M}}{N_{c}}\left\{\begin{array}{c}
\log _{2}\left(1+s n r_{0}\right), \text { no NLC } \\
\log _{2}\left(1+0.9 N_{c} / N_{M} s n r_{0}^{\frac{3}{2}}\right), \text { with } N L C
\end{array}\right.
$$

Where $N_{M}$ represents the level of multiplexing ( 1 for phase conjugate twin waves, and 2 for conjugate coding) snro represents the signal to noise ratio of a single copy of the signal without any form of nonlinearity compensation. The potential to improve the overall system performance of phase conjugate twin waves, and the generalization to multiple identical copies coherently added to the receiver, is shown in figure 26 (solid lines). When the impact of higher order parametric noise amplification is taken into account, there is little net improvement in the overall information spectral density, except for the lowest signal to noise ratio region. Polarization time coding, or phase conjugate coding provides compensation of the nonlinearity without the need to sacrifice bandwidth. The advantage of coding signals across the full bandwidth is clearly shown by the blue dashed curve in figure 26, where despite not improving the snr enhanced performance compared to phase conjugate twin waves is always observed.

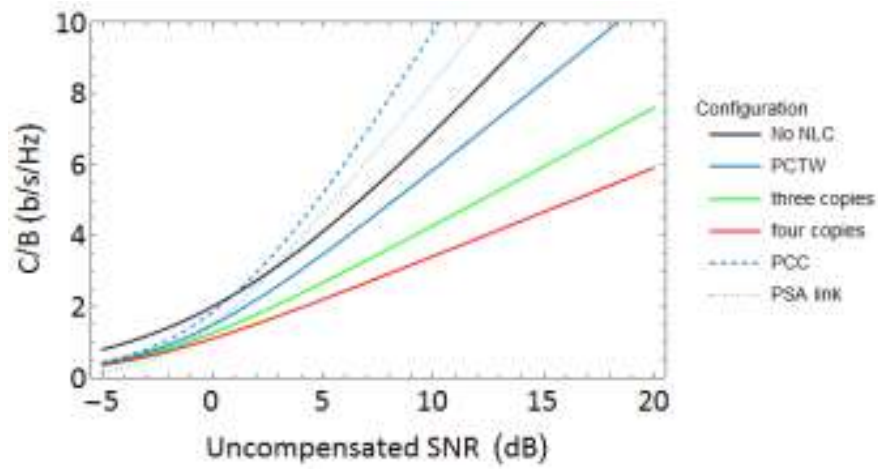

Figure 26: Comparison of maximum potential information spectral densities for transmission without nonlinearity compensation (black), transmission of two (blue), three (green) and four (red) identical copies (Two copies corresponding to phase conjugate twin waves), phase conjugate coding across two copies (blue dashed) and a dual wavelength phase sensitive amplifier link (blue dotted).

The phase conjugate twin wave scheme may be implemented in the optical domain by the use of four wave mixing devices to generate appropriate conjugate copies (usually in the wavelength domain) [261], simultaneously generating all of the required conjugate copies in a single device. At the receiver, a phase sensitive amplifier may be used to combine the original signal and the idler (the signals conjugate copy) through the inherent coherent addition associated with phase sensitive amplifiers [262]. Whilst such schemes do introduce their own complexities, such as pump phase locking [263] and strict requirements for dispersion management, in addition to the benefit of nonlinearity compensation, this scheme also benefits from the reduced noise figure of a phase sensitive amplifier. In the noise dominated region, this increases the resultant signal-to-noise ratio by $3 \mathrm{~dB}$, whilst providing a valuable $1.5 \mathrm{~dB}$ enhancement to the optimum signal to noise ratio albeit retaining the factor of two reduction in net available bandwidth. This is shown by the blue dotted curve in Figure 26, with the combination of improved snr and reduced nonlinear impairments result in potential increases in information spectral densities for systems were the original uncompensated signal to noise ratio (using $3 \mathrm{~dB}$ noise figure amplifiers) exceeds $3 \mathrm{~dB}$. Furthermore, the analysis presented here assumes that a phase sensitive link only compensates for intersignal nonlinearities (devices only inserted at the transmitter and receiver), however if PSAs are distributed throughout the transmission link, a reduction in the nonlinear impairments due to the interaction between signal and noise would also be expected, further enhancing the performance. This 
reduction in noise induced nonlinearity was be discussed in the context of optically phase conjugated links in section 5.2, and is expected to remain valid for a PSA link.

\subsection{The performance impact of imperfect compensation}

The theoretical discussions above assume perfect compensation, with arbitrary precision calculations and full knowledge of all system parameters. However, in practice, there are many features which disrupt the accuracy of nonlinearity cancellation, including practical component limitations, such as digital, optical or electrical signal processing bandwidth, digital resolution, and algorithm complexity to name a few of those where a tradeoff between cost and performance is possible to envision. Figure 27 generically shows the impact of finite nonlinear compensation efficiency on the maximum performance of a link. High compensation efficiency, typically exceeding $95 \%$, is required before the impact of the other terms in equations 25 and 33 have any significant impact. Below this value the compensated maximum signal to noise ratio is given, approximately, by $s n r_{\eta}=s n r_{0} \eta^{-1 / 3}$, where $\eta$ is the normalized residual nonlinearity. Above 95\% compensation efficiency it becomes necessary to include the impact of parametric noise amplification to accurately predict the performance and above $97.5 \%$, higher order terms. Importantly, for systems of finite compensation efficiency the simple inverse cubic relationship to predict the performance gain is perfectly valid.

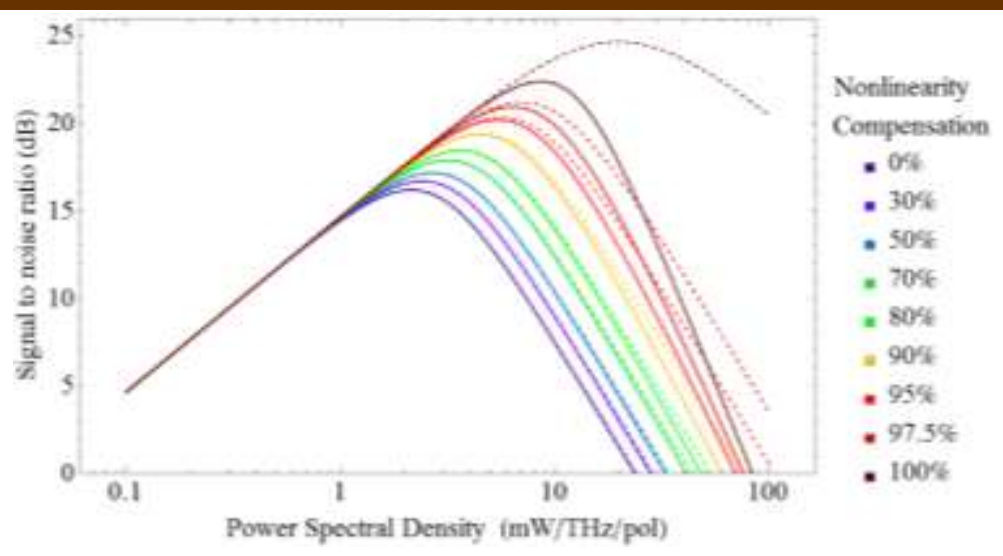

Figure 27. Theoretical nonlinear threshold characteristic for a 64 span system as a function of nonlinearity compensation efficiency considering residual inter-signal nonlinearity and first (dashed) and both first and second order (solid) parametric noise amplification.

Many factors impacting compensation efficiency are within the control of the system designer, and even power and dispersion symmetry can be predicted and either tracked in DSP, or for OPC systems controlled as described above [235]. However statistical polarization evolution experienced using a real transmission link will result in an unforeseeable asymmetry. This will limit the effectiveness of non-linear compensation possible [151] and has been reported as a significant restriction for the effectiveness of digital back propagation which is also constrained by the available signal processing bandwidth [206]. In essence, physical back propagation using OPC, and conventional DBP both assume that the relative polarization orientations of the different channels remain constant, and attempt to reverse the effects without adjustment of the polarization. Full statistical treatment of the restrictions placed on the effectiveness of a nonlinear compensator is complex, and a simple heuristic was proposed, based on the concept of a Lyot filter [161]. Here it was assumed that only that portion of the signal which would pass a pair of polarizers, one at the transmitter and one at the receiver ( of OPC) could possibly contribute to the nonlinear compensation. This simple approximation is useful for estimating when polarization mode dispersion will become a limiting feature, but in practice predictions based in its application to the nonlinear signal to noise ratio are only accurate to with around $1.5 \mathrm{~dB}$ and the full statistical treatment should be taken into account [245].

Fortunately, just as the linear impairments from polarization mode dispersion may be taken into account within the equalizer, so the degradation of nonlinearity compensation may be accounted for in the design of the compensator. In the case of digital back propagation it is, theoretically at least, possible to add a periodic polarization rotation stage to the back propagation algorithm. Conceptually it is readily argued that a polarization adjustment should ideally be applied at around half the polarization walk off length, with an increased frequency increasing the accuracy at the expense of complexity. For practical purposes, 
the ratio of polarization rotations to nonlinear steps should be a rational number, and whilst initial progress has been made by making this ratio an integer [264] or even unity [265] further optimization is required. In the case of OPC based links, it may be argued that provided the OPCs are spaced at less than half of the polarization walk off length, each adjacent segment between OPCs will have approximately identical polarization distributions for the channels, enabling effective nonlinearity compensation since the degree of random polarization rotation experienced by the signals before they are compensated is significantly reduced [245]. This assumption has been tested numerically using VPI TransmissionMaker 9.5 and Matlab. Five Nyquist shaped PM-QPSK channels were transmitted over thirty two $80 \mathrm{~km}$ spans of ideally Raman amplified fiber, to give a total transmission distance of over 2,560 km for various values of PMD. Typical results are shown in Figure 28.
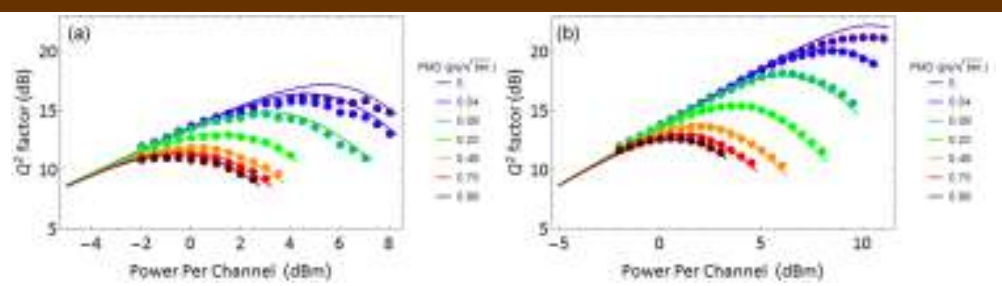

Fig 28: (a) :Analytically predicted (solid lines) and numerically simulated (dots) performance as a function of launch power per channel for a $2560 \mathrm{~km}$ system with one mid span OPC and different levels of PMD, from brown to blue PMD values are 1.0, 0.75, 0.5, 0.25, 0.1, 0.04, and 0 $\mathrm{ps} / \sqrt{\mathrm{km}}$. (2.2dB transmitter impairment). (b) As "a" but with OPC every two spans. There is no electronic nonlinearity compensation. Adapted from data set used for [245].

For this system, even modest levels of PMD, corresponding to state-of-the-art spun fiber, with PMD levels of $0.04 \mathrm{ps} / \sqrt{\mathrm{km}}$ have an observable impact. The performance gain is halved for $0.1 \mathrm{ps} / \sqrt{\mathrm{km}}$ PMD, and almost destroyed completely if PMD levels rise to $0.5 \mathrm{ps} / \sqrt{\mathrm{km}}$. Inclusion of $15 \mathrm{OPCs}$ significantly enhances the performance without PMD by the expected factor of $6 \mathrm{~dB}$, but more importantly in the presence of PMD increasing the number of OPCs also increases the performance markedly.

Solid lines in figure 28 show analytical fits including higher order parametric noise amplification, a $2.2 \mathrm{~dB}$ transmitter impairment and a compensation efficiency parameter determined by the mean PMD. Adapting [151] to calculate independently the nonlinear noise from each inter OPC segment gives an efficiency parameter for uniformly spaced OPCs of:

$$
\eta_{P M D}=\sum_{S=1}^{\frac{N_{a}}{N_{O P C}+1}} \frac{3\left(E i(-3 \xi)-E i\left(-3 \xi \alpha^{\prime 2} / \sigma_{N L}^{2}\right)\right)+E i(-7 \xi)-E i\left(-7 \xi \alpha^{\prime 2} / \sigma_{N L}^{2}\right)}{4 N_{a} L \log \left(\sigma_{N L} / \alpha^{\prime}\right)}
$$

where

$$
\xi=s B_{w}^{2} \frac{\pi^{3} L \sigma_{P M D}^{2}}{64}
$$

and where $\sigma_{P M D}$ is the PMD parameter $\alpha$ 'represents the inverse of effective span length which equals to $\alpha$ in case of lumped systems and equals to $1 /(N L)$ in case of ideal Raman systems, Ei(.) is the exponential integral, $\mathrm{N}_{\mathrm{OPC}}$ is the number of OPCs and $s$ is dummy variable equivalent to the span number within a given inter OPC segment. Good agreement is observed for all but the PMD free fiber, where the optimum performance is somewhat degraded. We believe that this is due to the imperfections in the DSP used in this simulation.

As shown in equation 27, the inter-signal nonlinear noise scales logarithmically with the signal bandwidth. The scaling factor may be expressed as $\ln \left(B / f_{w}\right)$ where $f_{w}^{-2}=2 \pi^{2}\left|\beta_{2}\right| L_{\text {eff }}$ and where Leffrepresents the conventional effective length of a single span for a lumped amplified system or the total length for an ideal lossless Raman amplified system. The majority of the nonlinear terms fall within the system bandwidth $B$ although a slightly broadening of the order of $f_{w}$ may be expected. For standard single mode fiber, $f_{w}$ is of the order of $10 \mathrm{GHz}$. If the compensated bandwidth $B_{c h}$ is large compared to $f_{w}$ and small compared to the overall WDM bandwidth $B_{s}$, which would be the case for super-channel propagation in a fully populated system, then we may split the nonlinear noise into terms falling within the signal processing bandwidth, and this falling outside this bandwidth. For most practical systems employing only digital nonlinearity compensators, the overall system bandwidth, all of which contributes something to the nonlinear noise, will greatly exceed the receiver bandwidth even if super-channel receivers (or 
frequency locked [266] and/or phase coherent transmitters [267]) are used. Furthermore, given that signal processing gains are finite, significant effort has been devoted to developing simplified nonlinearity compensators, based on digital filtering [225, 268], long and/or logarithmic step sizes [269], dominance of cross phase modulation, polarization and/or phase noise [207], Volterra series analysis [226] , neural networks [270] amongst others. In all cases, the compensator makes a reasonable approximation, but in doing so neglects some of the impact of nonlinearity. In many cases, the impact of the approximation is directly calculable. We consider first the signal bandwidth.

Considering inter-signal nonlinearities, and the first two orders of parametric noise amplification, the nonlinear noise summed over these two regions for a lumped amplifier system is then $[189,152]$;

$$
D_{N L}=N_{a}\left(\Gamma-\Gamma_{c h}\right) D_{S}^{3}+3\left(\frac{N_{a}\left(N_{a}+1\right)}{2} \Gamma-N_{a} \Gamma_{c h}\right) D_{S}^{2} D_{a}
$$

where the first term represents the impact of uncompensated inter-signal nonlinearity, the second term parametric noise amplification arising from signals within the compensator bandwidth (assuming split processing between transmitter and receiver) and the final term a first order estimation of the impact of the impact of uncompensated parametric noise amplification from the remainder of the signals. $\Gamma_{c h}$ represents the nonlinear scaling factor evaluated over the signal processing bandwidth. It is straightforward to show that, provided the signal processing bandwidth greatly exceeds $f_{w}$, the residual inter-signal nonlinearity scales as;

$$
\left(\Gamma-\Gamma_{c h}\right)=\left\{\begin{array}{cc}
\delta L_{e f f} \ln \left(\frac{B_{S}}{B_{c h}}\right), & \alpha>0 \\
\delta 2 L \ln \left(\frac{B_{S}}{B_{c h}}\right), & \alpha=0
\end{array}\right.
$$

Normalizing the residual scaling factor to the scaling factor without nonlinearity compensation gives a normalized residual nonlinearity factor $\eta_{D B P}=\left(\Gamma-\Gamma_{c h}\right) / \Gamma$.

Figures 29 and 30 shows the performance of four systems, each of 25 lumped amplified spans, with uncompensated signal to noise ratios ranging between 10 (purple) and $20 \mathrm{~dB}$ (red), and are plotted in terms of the absolute signal to noise ratio gain (figure 29), the relative improvement (the signal-to-noise ratio in decibels divided by the original signal to noise ratio in decibels) (figure 30) as functions of the residual nonlinearity factor (top row) and the aggregate DSP bandwidth (bottom row). In the limit of low compensation efficiency $\left(\eta_{D B P} \sim 1, B_{c h} \ll B_{S}\right)$ the absolute performance gain is relatively small reaching a few $\mathrm{dBs}$ only for large optical super-channels. Furthermore, as can be seen from Figure 29, the absolute gains in this region are almost independent of the system configuration, and are well approximated by the cube root of $\eta_{D B P}$. However, figure 30 shows that the improvement relative to the original snr is however strongly dependent on the system configuration for low compensation efficiency. On the other hand, in the limit of almost perfect compensation efficiency $\left(\eta_{D B P} \ll 1, B_{c h} \sim B_{S}\right)$, the relative improvement converges to the theoretical limit of around $50 \%$ of the initial signal-to-noise ratio in decibels (converging curves in figure 30), whereas the absolute gain (diverging curves in figure 29) is strongly dependent on the system configuration. However, to achieve this limit, signal processing bandwidths exceeding $99 \%$ of the signal bandwidth are required, along with accurate estimation of fiber parameters, including the polarization evolution. To achieve such high signal processing bandwidths, either very large superchannels are required $\left(B_{c h} \sim B_{S}\right)$ or a large number of independent channels should be jointly processed in some other way. It is unlikely that this later regime will be achieved over the full bandwidth allocated to communications without some form of optical signal processing, such as optical phase conjugation or phase sensitive amplification. 


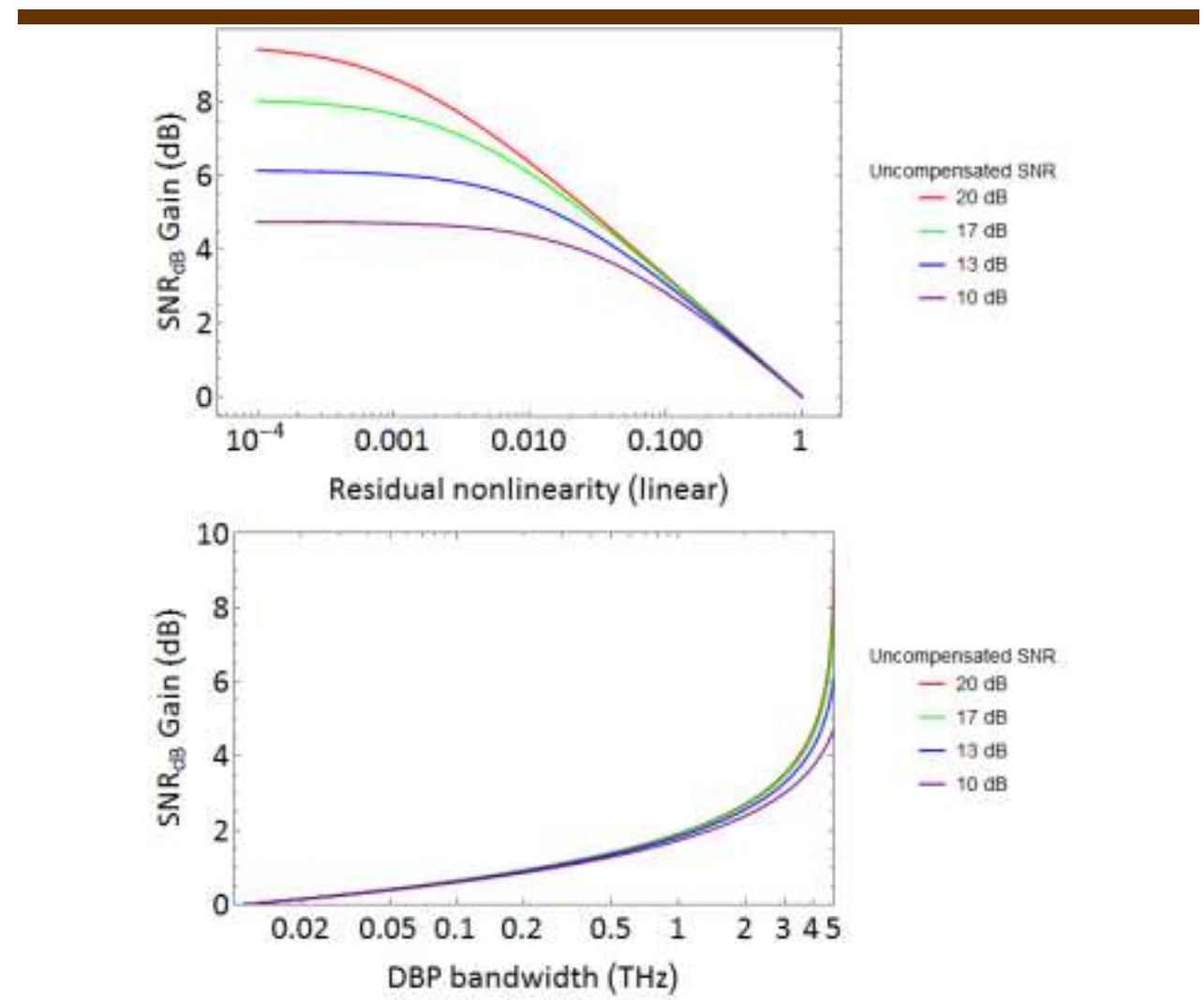

Figure 29: Analytically predicted maximum performance gain for a selection of 25 span transmission systems, each with a total bandwidth of $5 \mathrm{THz}$ and uncompensated signal to noise ratios of 20 (red), 17 (green), 13 (blue) and 10 (purple) dB showing the performance versus compensation efficiency (top row) and digital signal processing bandwidth (bottom row) in terms of absolute performance gain (left) and the performance gain normalised to the original signal to noise ratio. Concept adapted from [187].

For a system employing ideal OPC, such high bandwidth performance is possible [254] and although care should be taken to ensure path and polarization matching if diverse schemes are used for either polarization or waveband diversity, the full anticipated performance gain should be possible. In hybrid systems, where DSP is used to further suppress parametric noise amplification or to account for penalties from symmetry, a bandwidth dependence will be present from the parametric noise amplification terms, however this is a much more modest variability than with DSP alone, where the finite bandwidth impacts on the stronger inter-signal nonlinearity. Similar arguments apply to transmit side and split DSP, where some slight mitigation of parametric noise amplification may be observed. 


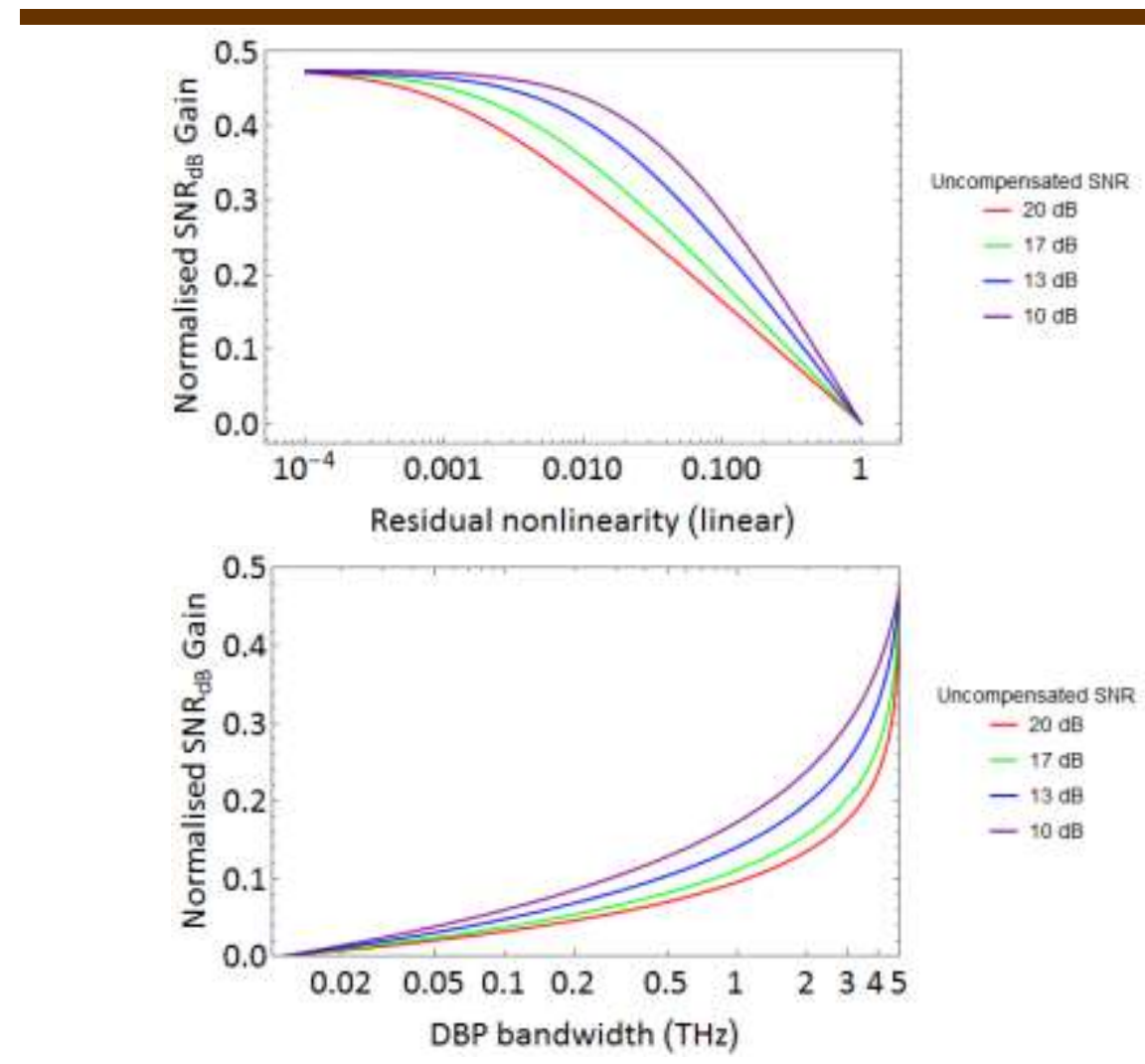

Figure 30: Analytically predicted maximum performance gain for a selection of 25 span transmission systems, each with a total bandwidth of $5 \mathrm{THz}$ and uncompensated signal to noise ratios of 20 (red), 17 (green), 13 (blue) and 10 (purple) dB showing the performance versus compensation efficiency (top row) and digital signal processing bandwidth (bottom row) in terms of absolute performance gain (left) and the performance gain normalised to the original signal to noise ratio.

For numerical simulations and laboratory demonstrations, uniform fiber lengths are often assumed. This gives rise to the opportunity to maximize the symmetry for OPC (see Equation 36), and the fully determine the nonlinear effects for DSP based nonlinearity compensators. However, with the possible exception of long haul submarine systems, practical communication networks do not enjoy such high levels of uniformity. Where the amplifier spacing is variable, the resonantly enhanced (quasi phase matched) peaks associated with four-wave mixing with uniformly spaced fibers are effectively washed out, and the contribution to the total nonlinear noise of each span are simply added as independent random variables. Considering inter-signal nonlinearity only for simplicity, the inter-signal contribution to the nonlinear noise becomes

$$
D_{N L}=\sum_{i=1}^{N_{a}} \Gamma_{i} D_{S i}^{3}
$$

with the nonlinear scaling parameter $\Gamma_{i}$ varying in principle from span to span and determined by the fiber parameters of the $i^{\text {th }}$ span at the noise accumulated including any amplified spontaneous emission noise generated during of immediately after the fiber transmission. The signal power spectral density $D_{S i}$ may take a constant value for each span, however it is straight forward to show that the total nonlinear noise density is most readily minimized by optimizing the launch conditions on a span-by-span basis, and optimization strategy also known as Local Optimization for Global Optimization [271-272]. Adaptive digital signal processing is then required in order to track the fiber parameters and launch power spectral densities of each span, with knowledge of approximate fiber designations and lengths allowing equation 45 to be used to provide initial estimates of the necessary parameters.

For OPC the widespread conception is that such non-uniformity would destroy the compensation. However, this is not strictly the case [230, 273-274]. Whilst it is likely that non-uniform spans would prevent an OPC system reaching the limit imposed by parametric noise amplification, achievement of a 
few $\mathrm{dB}$ gain over all channel simultaneously remains a reasonable option. Full analysis of the four-wave mixing process reveals the impact of differences in dispersion in such circumstances to be tolerable, whilst numerical simulations have revealed the modest impact of non-central OPC placement and that much of this impact may be recovered using DSP. Recent experimental investigations over field installed fiber $[247,252]$ have also suggested that the impact of asymmetry may also be addressed through appropriate dispersion management, in analogy to dispersion compensated spectral inversion. Further development in the understanding of imperfect systems is also likely to result in additional proposals to mitigate their impact.

Finally, for all nonlinear compensation strategies, the compensator itself may add additional signal degradations. For electronic signal processing, finite effective number of bits, component frequency responses and linearity, step size, and sampling rate may all contribute to degradations of signal quality, whilst for OPC based systems, the trade-off between additional inter-signal nonlinearity and finite conversion efficiency may lead to some additional degradation. All of these issues have impacted experimental measurements (see tables 2 and 3 ) but in principle may be eliminated following sufficiently concerted engineering effort.

\section{Nonlinear scattering effects.}

The majority of recent attention in understanding the performance limits of optical fiber communication systems has focused on the Kerr nonlinearity (see sections 3.2 and 4.2 above). In addition to this nonlinearity based on an interaction between the optical field and bound electrons within the medium, there are also interactions between the optical field and vibrational modes (phonons) of the medium. These are typically split into longitudinally propagating acoustic phonons (the Brillouin effect), transversely propagating optical phonons (the acousto-optic effect) and optical phonons (forward and backwards Raman Effect) [275].

Stimulated Brillouin Scattering (SBS) acted as an effective limit on the maximum signal launch power for non-return-to-zero amplitude modulated systems where half the power resided in the carrier [,276, 277]. SBS threshold per channel was found to be independent of the number of channels, owning to the narrow gain bandwidth [278]. Suppression of SBS was an important feature of amplified transmission systems using NRZ modulation, and was achieved by intentionally or implicitly including a time varying modulation of the phase angle of the light waves [279]. A more direct approach is to adopt frequency [280281], phase [277, 281, 282] or duobinary [283] modulation, where the power is spread more uniformly across the signal bandwidth, without a strong carrier component. In effect, the signal power is spread over a bandwidth much greater than the SBS linewidth, leading to a significant increase in the threshold. A typical SBS threshold is around $+7 \mathrm{~dB}$ of continuous wave power, and a typical gain bandwidth, $10 \mathrm{MHz}$. Research on suppressing SBS [277-283] suggests that penalties begin to accumulate when the signal power spectral density exceeds $500 \mathrm{~mW} / \mathrm{GHz}$. This comfortably exceeds, by several orders of magnitude, the predicted optimum signal power spectral densities for systems without nonlinearity compensation of around $100 \mu \mathrm{W} / \mathrm{GHz}$. Systems employing optical phase conjugation to compensate for nonlinearity suggest that the signal launch power may be increased by between 10 and $20 \mathrm{~dB}$ (Figure 20), given the constraints of transmitter signal to noise ratio [284]. The resultant power level would still remain a little below the SBS threshold, but would potentially be sufficiently close to cause difficulties if modulator biases were allowed to drift, resulting in finite continuous wave components.

The closely related acousto-optic effect, or guided acoustic wave Brillouin scattering (GAWBS) causes a long range interaction, and is responsible for phase and arrival time changes in optical pulse sequences $[84,285]$. Whilst the impact of the acousto-optic effect is increased for higher bit rate (shorter pulse) systems, suggesting that it may be significant for broadband signals (see section 3.2), in fact it scales very gently with the number of channels and that "wavelength division multiplexing ... does not increase considerably the bit error rate due to electrostrictional interaction" [84].

The Raman Effect [286] also comes in two flavors, in this case forwards and backwards effects. The forward effect is responsible for nonlinear effects such as the soliton self-frequency shift [83] and contributes to optical rogue wave generation $[287,288]$, whilst both forwards and backwards effect are responsible for power transfer between channels, acting as an additional loss mechanism for short wavelength signals, along with pump mediated crosstalk for systems employing Raman amplification [286, 289-290]. In detail, the Raman gain profile $g_{R}$ is quite complex, but is often analyzed with a simple triangular profile $g_{R}(\Delta f)=g_{R \max } \Delta f / \Delta f_{\max }$, where $g_{R \max }$ is the maximum gain at a detuning of $\Delta f_{\max }$ and the detuning between two specific signals is $\Delta f$ [290-291]. Denoting the signal power spectral density of the $i^{\text {th }}$ frequency component of a WDM signal with a total bandwidth of $B_{S}$ as $D_{S(i)}$ the mean power spectral density evolution is given approximately by [292-293]; 


$$
\frac{\partial D_{S(i)}}{\partial z}=-\alpha D_{S(i)}+\sum_{j=1, j \neq i}^{B_{S} / B_{c h}} g_{R}\left(B_{c h}(j-i)\right) D_{S(i)} D_{S(j)}
$$

Which has been extensively used to predict the performance of multi-pump Raman amplifier systems. Figure 31 illustrates the impact of equation 45 on the output signal power spectral density for a $100 \mathrm{~km}$ span with an input power spectral density of $0.5 \mathrm{~mW}$ per $33 \mathrm{GHz}\left(1.510^{-14} \mathrm{~W} / \mathrm{Hz}\right)$, close to the optimum power given by the trade-off between amplified spontaneous emission noise and the interaction between signals mediated by the Kerr effect. In the absence of nonlinearity compensation, gain tilts of approximately $\pm 2 \mathrm{~dB}$ would be expected, induced by the Raman Effect and is considered an important design parameter for wideband systems. For systems employing conventional Erbium doped amplification, the gain tilt is significantly reduced, to less than $\pm 0.5 \mathrm{~dB}$ and is typically neglected. This gain tilt will have an impact not only on the optical signal-to-noise ratio for short wavelength channels, but also the effective length of the Kerr effect for longer wavelength signals, degrading the nonlinear signal to noise ratio. Optimum pre-emphasis should be designed to mitigate both of these effects simultaneously.

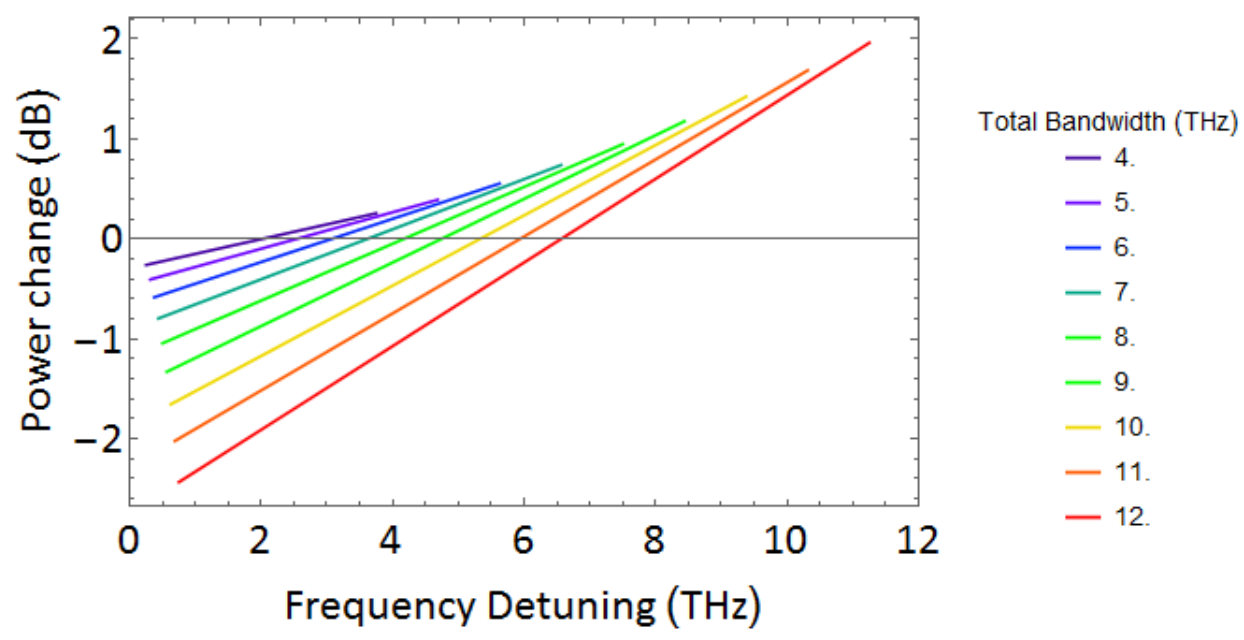

Figure 31: Approximate impact of inter-signal Raman power transfer over a $100 \mathrm{~km}$ span as a function of channel position for total spectral widths of between 4 (purple) and 12 (red) THz, assuming a Raman gain coefficient of $0.3 / \mathrm{W} / \mathrm{km}$ at a peak signal separation of $12 \mathrm{THz}$, attenuation of $0.2 \mathrm{~dB} / \mathrm{km}$ and a launched signal power spectral density of $-3 \mathrm{dBm}$ per $33 \mathrm{GHz}$ channel bandwidth.

In addition to gain tilt which may be readily compensated, amplitude modulated signals produce an additional source of noise for the longer wavelength signals [275] in any given system, and has been thoroughly analyzed for on-off keyed intensity modulated signals [294]. As with the Kerr effect, walk-off between channels has a significant effect [295], but unlike the Kerr effect, the resultant penalties are strongly dependent on the channel frequency [296]. One study [294] suggests that in the absence of walkoff the nonlinear noise variance is approximately equal to the change in signal power. Thus the $1 \mathrm{~dB}$ power changes typically observed in Figure 31 would therefore correspond to a signal-to-noise ratio of only $5.8 \mathrm{~dB}$ and significant transmission penalties. For a high dispersion fiber however, the noise variance is significantly reduced, by the ratio of the dispersion length (of the entire WDM system) to the effective length [294]. This results in a net penalty of less than one hundredth of a $\mathrm{dB}$ per span, even for a $12 \mathrm{THz}$ wide WDM signal, consistent with recent experimental observations of the validity of the Gaussian noise model for bandwidths up to $7.3 \mathrm{THz}$ [297], and suggesting that Raman induced nonlinear noise may be neglected. However, if nonlinearity compensation is employed to mitigate the impact of the Kerr effect, the optimum signal power will increase substantially, with over $95 \%$ depletion of the lowest wavelength channel occurring after the launch power spectral density for a $12 \mathrm{THz}$ bandwidth system is increased by 
more than $\mathrm{x} 4$ (x30 for a $4 \mathrm{THz}$ bandwidth system), suggesting that the small signal approximations of [294] may not be valid in the regime of nonlinearity compensated systems and a more detailed study of Raman crosstalk may be required.

Overall, scattering nonlinearities do not appear to have a significant impact on the statistical properties of the received signals beyond a gain tilt, unless both nonlinearity compensation and ultra-wide bandwidth amplifiers are employed.

\section{Network Implications}

Whilst potential capacity improvements for a point to point system may be readily calculated as shown above, for an optical network a wide variety of link lengths are presented, often with multiple routes sharing the same optical fiber. Not all routes would fully benefit from the available nonlinearity compensation, especially bearing in mind that OPCs may not be symmetrically placed, and that the technical difficulties of ultra-broadband nonlinearity compensation using DSP limit the capacity gains Calculations of snr gain based on point to point links will therefore inevitably be somewhat optimistic. However, it is revealing to consider the maximum possible benefits of nonlinearity compensation and here we illustrate the potential benefit for a wide variety of networks. We consider a simple heuristic model which has proven to be sufficiently accurate for network resource calculation purposes [298], based on widely available geographic area and population data [299], and further assume one core exchange location per 3.5 million people. For each link length predicted by the network model [300] we calculate the maximum potential snr performance relative to a polarization multiplexed 16-QAM system with a reach of $800 \mathrm{~km}$ assuming (a) no nonlinearity compensation, (b) digital back propagation with a maximum performance gain of $1.2 \mathrm{~dB}$, (c) nonlinear compensation corresponding to each signal passing through a single OPC, and (d) and OPC placed at every amplifier site. For OPC systems, a performance gain of $1.2 \mathrm{~dB}$ is applied if the link length is less than or equal to two amplified spans. This is in turn converted to the maximum capacity of each link by determining the maximum transmittable QAM modulation format (in steps of $0.5 \mathrm{~b} / \mathrm{s} / \mathrm{Hz} / \mathrm{pol}$, assuming Trellis coding) by rounding the delivered signal to noise ratio down. Figure 32 illustrates the predicted distribution of modulation formats for two countries, Mexico and Brazil. In both cases, as expected, the $1.2 \mathrm{~dB}$ gain from DBP only offers marginal improvement in the capacity of each link. On the other hand, the most common information spectral density (Mode) increases from 6 to 10.5 for the Mexico and from 4 to 9.5 in Brazil. In both cases, the most common ISD with multiple OPC exceeds the maximum ISD without nonlinearity compensation. Indeed, the two distributions have almost zero overlap. A critical observation is therefore that extensive OPC deployment will only realize its full potential if it is accompanied by significant upgrades in transponder capabilities. Note, for densely populated small countries, such as the UK, the multiple OPC curve shows two peaks due to the high number of single span links, never-the-less the need for higher order modulation formats remains.
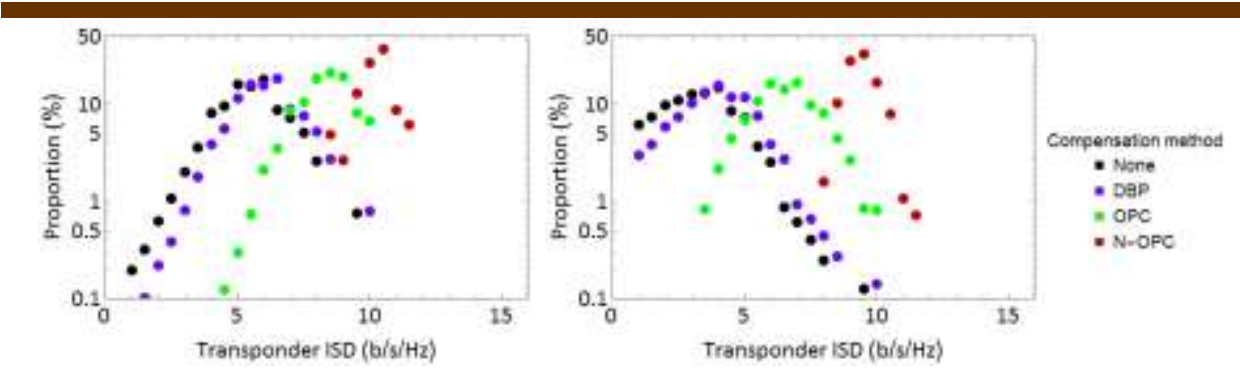

Figure 32: Analytically predicted distribution of modulations formats for Mexico (left) and Brazil (right), assuming flexible modulation formats with a resolution of $0.5 \mathrm{~b} / \mathrm{s} / \mathrm{Hz}$ obtained through coding, and no nonlinearity compensation (Black), simple electronic compensation offering a $1.2 \mathrm{~dB}$ performance gain (blue), an OPC at the midpoint of each link (green), and an OPC every span (minimum span length $40 \mathrm{~km}$, two spans required for OPC deployment). Adapted from [298].

The link capacities may be used to calculate the total network capacity, and the inverse of the network capacity, assuming an arbitrarily large total capacity demand (projected say 10 years beyond the "capacity crunch"), may be used in turn to estimate the number of parallel fiber systems which would be required, as shown in Figure 33. Here we observe that digital nonlinearity compensation has negligible impact on the total number of fibers required for a post "capacity crunch" network, with the national network with 
the most amenable conditions is predicted to enjoy a reduction in fiber count to only $86 \%$ of that required without nonlinearity compensation. For small populations, supporting only a few network nodes, a similarly disappointing benefit is observed however, for larger populations OPC offers the prospect of reducing the total fiber count to $50 \%$ of the requirement without nonlinearity compensation, or even as low as $25 \%$ if multiple OPCs are used in appropriate networks, such as Russia, Brazil, Canada where the ratio of linear network size to population served is large. More formal studies of network enhancement for digitally compensated networks [301] and networks employing optical phase conjugation [302], which consider realistic traffic matrices and accurate link distributions, have shown for a small number of test cases that the majority of the benefits predicted by the simple approach (that is, increases of network capacity of between $25 \%$ and $100 \%$ ) may be realistically expected. The particular advantage of nonlinearity compensation over alternative approaches such as space division multiplexing [303] will depend greatly on the prevailing economic conditions. For example, in a submarine system constrained by the size of the repeater, inclusion of nonlinearity compensation results in record per fiber capacities [304]. On the other hand, for an energy constrained system cable capacity is enhanced by reducing the per carrier modulation formal below the nonlinear threshold [305].
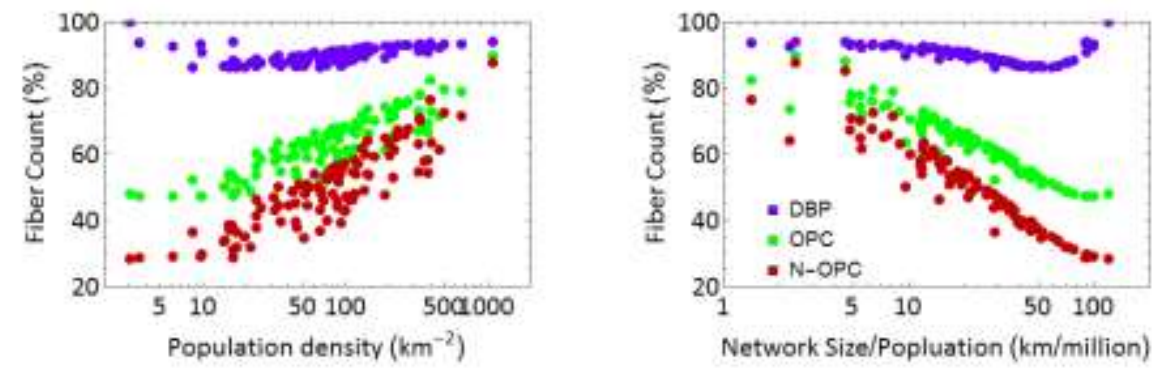

Figure 33. Estimate of economic benefit of nonlinearity compensation as a function of network parameters (assuming national scale networks) for massive capacity optical networks, plotted as a function of population density served (left) and as a function of the ratio between network scales to population (right)

\section{Conclusions}

In this paper we have reviewed the progress in calculation of the maximum performance of a single mode optical fiber, from the first shot noise limit calculations through to the accepted limits of today. Many of the original calculations are still relevant today, especially for cost sensitive applications where fully featured coherent detection is often avoided. In section 5 we discovered that for coherent transmission systems the originally conceived nonlinear Shannon limit may be readily overcome by compensating inter-signal nonlinearity, only to be replaced by a limit imposed by the nonlinear interaction between signal and noise, and that to calculate this limit, some of the original assumptions need to be revisited. Recent reports have also suggested that optimized coding coupled advanced nonlinearity compensation may even allow some mitigation of this limit. Such advances have cast doubt onto the truly fundamental nature of the originally coined nonlinear Shannon limit. These doubts are reasonable given the number of assumptions and approximations built into the deviation of the various forms of the limit, and how readily these assumptions are breached once compensation is implemented. However research into nonlinearity compensation makes two factors abundantly clear. Firstly, stochastic and practical imperfections in system design (eg PMD and DSP resolution respectively) will significantly limit the gains unless multiple OPCs are used. Secondly, since the system throughput only depends logarithmically on the signal to noise ratio and it have been shown that the (linear) Shannon limit remains an upper bound even for nonlinear transmission systems [306], the performance of an optical fiber communication system is fundamentally limited by nonlinearity and noise.

At the time of writing, even though the actual in-line system only accounts for a few $\%$ of the total energy consumption of a link, exponentially increasing the launch power in order to linearly increase system throughput will eventually lose its appeal - even if it were possible. However, a practical upper bound on the launch power is imposed by the various mechanisms for fiber damage, and although new fiber designs 
and improved coatings have an influence on this limit [307-308] it remains finite. So even from the simple view point of a practical maximum launch power [11] and the linear Shannon limit, and only considering shot noise [309], we must inevitably accept that the capacity of an individual fiber is finite, and that in order to maintain growth in service provision, corresponding growth in parallel transmission systems is inevitable.

Funding. This work is partially funded by; the Engineering and Physical Sciences Research Council (EPSRC) (EP/J017582/1-UNLOC, EP/L000091/1-PEACE, EP/M005283/1-UPON); the Royal Society Wolfson Research Merit Award Scheme (WM120035). The authors would like to thank M. Tang, W. Forysiak, T. Zhang, M. Sorokina and S. Sygletos for useful discussions.

\section{REFERENCES}

1. J-C. Antona, "Key technologies for Present and Future Optical Networks," presented at Topical Workshop on Electronics for Particle Physics Plenary Session 5, Paris, France, 21-25 September 2000 (URL: https://indico.cern.ch/event/49682/contribution/154).

2. A.D. Ellis, J. Zhao, and D. Cotter, "Approaching the Non-Linear Shannon Limit," Journal of Lightwave Technology, 28(4), 423-433 (2010).

3. A.D. Ellis, N. Mac Suibhne, D. Saad, and D.N. Payne, "Communication networks beyond the capacity crunch,” Phil. Trans. R. Soc. A, 374(2062), 20150191 (2016).

4. D.J. Richardson, "Filling the Light Pipe," Science 330(6002), 327-328 (2010).

5. A. Chraplyvy. "The Coming Capacity Crunch," in proceedings of European Conference on Optical Communications, (IEEE, 2009) Second Plenary Presentation. (http://ieeexplore.ieee.org/stamp/stamp.jsp?tp=\&arnumber=5287305\&isnumber=5286960)

6. The Editor, "First non-military fibre-optic link," Electronics and Power 22, 285 (1975). (URL: http://ieeexplore.ieee.org/stamp/stamp.jsp?tp=\&arnumber=5183663)

7. R.S.Tucker, "Green Optical Communications-Part I: Energy Limitations in Transport," Journal of Selected Topics in Quantum Electronics, 17, 245-260 (2011).

8. G.Patterson, "BT Group plc Annual Report 2015," https://www.btplc.com/Sharesandperformance/Annualreportandreview/pdf/2015_BT_Strategi c Report.pdf

9. R. J. Essiambre and R. W. Tkach, "Capacity Trends and Limits of Optical Communication Networks," Proceedings of the IEEE, 100(5), 1035-1055 (2012).

10. D. D. Matulka, "Application of LASERS to Digital Communications," IRE Transactions on Aerospace and Navigational Electronics, ANE-9(2), 104-109 (1962).

11. K. C. Kao and G. A. Hockham, "Dielectric-fibre surface waveguides for optical frequencies," Proceedings of the Institution of Electrical Engineers, 113(7), 1151-1158 (1966).

12. . K. Willox, "Q factor: the wrong answer for service providers and equipment manufacturers," in IEEE Communications Magazine, 41(2), S18-S21 (2003).

13. J.G. Proakis, Digital communications, (McGraw-Hill, 1995)

14. R.A. Shafik, M. S. Rahman, A.H.M.R. Islam, "On the extended relationships among EVM, BER and SNRas Performance Metrics," In Proceedings of $4^{\text {th }}$ International Conference on Electical and Computer Engineering, ICECE 2006 (IEEE, 2016), 408-411.

15. R. Schmogrow, B. Nebendahl, M. Winter, A. Josten, D. Hillerkuss, S. Koenig, and J. Meyer, "Error vector magnitude as a performance measure for advanced modulation formats," IEEE Photonics Technology Letters 24(1), 61-63 (2012)

16. A. Alvarado, E. Agrell, D. Lavery, R. Maher, and P. Bayvel, "Replacing the Soft-Decision FEC Limit Paradigm in the Design of Optical Communication Systems," J. Lightwave Technol. 34, 707-721 (2016)

17. N. S. Kapany and J. J. Burke, "Fiber Optics. IX. Waveguide Effects," J. Opt. Soc. Am. 51, 1067-1078 (1961).

18. O. E. DeLange, "Wide-band optical communication systems: Part II-Frequency-division multiplexing," Proceedings of the IEEE, 58(10), 1683-1690 (1970).

19. F.P. Kapron, D. B. Keck, and R. D. Mauer, "Radiation losses in glass optical waveguides", Appl. Phys. Lett., 17B(10), 423-425 (1970)

20. T. Miya, Y. Terunuma, T. Hosaka, and T. Miyashita, "Ultimate low-loss single-mode fibre at $1.55 \mu \mathrm{m}$," Electronics Letters, 15(4), 106-108 (1979).

21. G. Fillmore and G. Lachs, "Information rates for photocount detection systems," IEEE Transactions on Information Theory, 15(4), 465-468 (1969).

22. T. S. Kinsel, "Wide-band optical communication systems: Part I-Time division multiplexing," Proceedings of the IEEE, 58(10), 1666-1683 (1970). 
23. H. Steinberg, "The use of a laser amplifier in a laser communication system," Proceedings of the IEEE, 51(6), 943-943 (1963).

24. R.C. Hooper, D.B. Payne, and M. H. Reeve, "The Development of Single-Mode Fibre Transmission Systems at BTRL," Journal of the Institution of British Telecommunications Engineers, 4(2), 74-78 (1985).

25. R.H. Wentworth, G.E. Bodeep, and T.E. Darcie, "Laser mode partition noise in lightwave systems using dispersive optical fiber," Journal of Lightwave Technology 10, 84-89 (1992).

26. A. Lord, L. C. Blank, S. F. Carter, M. J. O'Mahony, S. J. Pycock, D. M. Spirit, and J. V. Wright "Linear Propagation Effects," in High capacity optical transmission explained D.M. Spirit and M.J. O"Mahony eds, (Wiley, 1995)), 61-88.

27. N. A. Olsson, "Lightwave systems with optical amplifiers," Journal of Lightwave Technology, 7(7), 1071-1082 (1989).

28. S. Walklin and J. Conradi, "Multilevel signaling for increasing the reach of $10 \mathrm{~Gb} / \mathrm{s}$ lightwave systems," Journal of Lightwave Technology, 17(11), 2235-2248 (1999).

29. A. Naka and S. Saito, "In-Line Amplifier Transmission Distance Determined by Self-phase Modulation and Group-Velocity Dispersion," Journal of Lightwave technology, 12(2), 280287 (1994).

30. G.P.Agrawal, Nonlinear Fiber Optics, Second Edition, (Academic Press, 1995).

31. R.S. Vodhanel, A. F. Elrefaie, M. Z. Iqbal, R. E. Wagner, J. L. Gimlett, and S. Tsuji, "Performance of directly modulated DFB lasers in 10-Gb/s ASK, FSK, and DPSK lightwave systems," Journal of lightwave technology 8(9), 1379-1386, (1990).

32. A.D.Ellis, "All optical networking beyond 10 Gbits/s: OTDM networks based on electro-optic modulators and fibre ring lasers", $\mathrm{PhD}$ thesis (Aston University, 1997).

33. M. Tan, P. Rosa, S. T. Le, Md. A. Iqbal, I. D. Phillips, and P. Harper, "Transmission performance improvement using random DFB laser based Raman amplification and bidirectional second-order pumping," Opt. Express 24, 2215-2221 (2016).

34. K. Zou; Y. Zhu; F. Zhang, "800Gb/s $(8 \times 100 \mathrm{~Gb} / \mathrm{s})$ Nyquist Half-Cycle Single Sideband Modulation Direct Detection Transmission over 320km SSMF at C-band," Journal of Lightwave Technology (to be published).

35. B. Zhu, J. Zhang, J. Yu, D. Peckham, R. Lingle, M.F. Yan, and D.J. DiGiovanni, " 34.6 Tb/s $(173 \times 256 \mathrm{~Gb} / \mathrm{s})$ single-band transmission over $2400 \mathrm{~km}$ fiber using complementary Raman/EDFA," in Optical Fiber Communication Conference, OSA Technical Digest Series (Optical Society of America, 2016), paper Tu3A1.

36. S. Zhang, F. Yaman, Y.K. Huang, J.D. Downie, D. Zou, W.A. Wood, and J. Hurley, "Capacity-Approaching Transmission over $6375 \mathrm{~km}$ at Spectral Efficiency of $8.3 \mathrm{bit} / \mathrm{s} / \mathrm{Hz}$,", ," in Optical Fiber Communication Conference, OSA Technical Digest Series (Optical Society of America, 2016), paper Th5C2.

37. J. Cai, H. G. Batshon, M. Mazurczyk, O. V. Sinkin, D. Wang, M. Paskov, W. Patterson, C. Davidson, P. Corbett, G. wolter, T. Hammon, M. A. Bolshtyansky, D. Foursa, and A. Pilipetskii, "70.4 Tb/s Capacity over 7,600 km in C+L Band Using Coded Modulation with Hybrid Constellation Shaping and Nonlinearity Compensation," in Optical Fiber Communication Conference, OSA Technical Digest Series (Optical Society of America, 2017), paper Th5B.2.

38. A. Amari, P. Ciblat and Y. Jaouën, "Inter-Subcarrier Nonlinear Interference Canceler for Long-Haul Nyquist-WDM Transmission," IEEE Photonics Technology Letters, 28(23), 27602763 (2016).

39. S. Saito, M. Murakami, A. Naka, Y. Fukada, T. Imai, M. Aiki, and T. Ito, "Inline amplifier transmission experiments over $4500 \mathrm{~km}$ at $2.5 \mathrm{~Gb} / \mathrm{s}$," Journal of Lightwave Technology, 10(8), 1117-1126 (1992).

40. C. Caspar, H. -M. Foisel, A. Gladisch, N. Hanik, F. Kuppers, R. Ludwig, A. Mattheus, W. Pieper, B. Strebel, and H. G. Weber, "RZ versus NRZ modulation format for dispersion compensated SMF-based 10-Gb/s transmission with more than 100-km amplifier spacing," IEEE Photonics Technology Letters, 11(4), 481-483 (1999).

41. N. S. Bergano and C. R. Davidson, "Circulating loop transmission experiments for the study of long-haul transmission systems using erbium-doped fiber amplifiers," Journal of Lightwave Technology, 13(5), 879-888 (1995).

42. C. Lorattanasane and K. Kikuchi, "Parametric instability of optical amplifier noise in longdistance optical transmission systems," IEEE Journal of Quantum Electronics, 33(7), 10681074 (1997)

43. R. Hui, M. O'Sullivan, A. Robinson, and M. Taylor, "Modulation instability and its impact in multispan optical amplified IMDD systems: theory and experiments," Journal of Lightwave Technology, 15(7), 1071-1082 (1997). 
44. D. Malyon and T. Widdowson, "2.5 Gbit/s NRZ system aspects for transoceanic distances," Electronics Letters, 28(16), 1529-1531 (1992).

45. D. Marcuse, "Single-channel operation in very long nonlinear fibers with optical amplifiers at zero dispersion," in Journal of Lightwave Technology, 9(3), 356-361 (1991).

46. Y. Miyamoto, T. Kataoka, A. Sano, T. Ono, K. Hagimoto, K. Aida, and Y. Kobayashi, "10Gbit/s 280-km nonrepeatered transmission with suppression of modulation instability," in Optical Fiber Communication Conference, 4 of 1994 OSA Technical Digest Series (Optical Society of America, 1994), paper TuN2.

47. B. Mikkelsen, G. Raybon, R. J. Essiambre, J. E. Johnson, K. Dreyer and L. F. Nelson, "Unrepeatered transmission over $150 \mathrm{~km}$ of nonzero-dispersion fibre at $100 \mathrm{Gbit} / \mathrm{s}$ with semiconductor based pulse source, demultiplexer and clock recovery," Electronics Letters, 35(21), 1866-1868 (1999).

48. H. Taga, N. Edagawa, Y. Yoshida, S. Yamamoto, M. Suzuki, and H. Wakabayashi, "10 Gbit/s, 4500km Transmission Experiment Using 138 Cascaded Er-doped Fiber Amplifiers," ," in Optical Fiber Communication Conference, 5 of 1992 OSA Technical Digest Series (Optical Society of America, 1992), paper PD12.

49. M. Nakazawa, T. Yamamoto and K. R. Tamura, "1.28 Tbit/s-70 km OTDM transmission using third- and fourth-order simultaneous dispersion compensation with a phase modulator," in Electronics Letters, 36(24), 2027-2029 (2000).

50. S. Saito, M. Murakami, A. Naka, Y. Fukada, T. Imai, M. Aiki, and T. Ito, "Inline amplifier transmission experiments over $4500 \mathrm{~km}$ at $2.5 \mathrm{~Gb} / \mathrm{s}$," Journal of Lightwave Technology, 10(8), 1117-1126 (1992).

51. M.Chagnon, M. Osman, M. Poulin, C. Latrasse, J.-F. Gagné, Y. Painchaud, Carl P., S. Lessard, and D. Plant. "Experimental study of $112 \mathrm{~Gb} / \mathrm{s}$ short reach transmission employing PAM formats and SiP intensity modulator at $1.3 \mu \mathrm{m}$." Optics express 22(17), 21018-21036 (2014).

52. E. El-Fiky, M. Chagnon, M. Sowailem, A. Samani, M. Morsy-Osman, and D.V. Plant, "168 Gb/s Single Carrier PAM4 Transmission for Intra Data Center Optical Interconnects," IEEE Photonics Technology Letters, 29(3), 314-317 (2017).

53. K. Zhong, X. Zhou, Y. Wang, Y. Wang, W. Zhou, W. Chen, and C. Lu, 2Transmission of a 120-GBd PM-NRZ Signal Using a Monolithic Double-Side EML," IEEE Photonics Technology Letters, 28(20), 2176-2179 (2016).

54. H. Yamazaki, M. Nagatani, F. Hamaoka, S. Kanazawa, H. Nosaka, T. Hashimoto, and Y. Miyamoto, “ 300-Gbps Discrete Multi-tone Transmission Using Digital-Preprocessed AnalogMultiplexed DAC with Halved Clock Frequency and Suppressed Image," in Proceedings of $42^{\text {nd }}$ European Conference and Exhibition of Optical Communication (VDE, 2016), 25-27.

55. S. Kanazawa, H. Yamazaki, Y. Nakanishi, T. Fujisawa, K. Takahata, Y. Ueda, and H. Sanjoh, "Transmission of 214-Gbit/s 4-PAM signal using an ultra-broadband lumped-electrode EADFB laser module". in Optical Fiber Communication Conference, OSA Technical Digest Series (Optical Society of America, 2017), paper Th5B-3.

56. K. Zhong, X. Zhou, Y. Gao, W. Chen, J. Man, L. Zeng, and C. Lu, “140 Gbit/s 20km transmission of PAM-4 signal at $1.3 \mu \mathrm{m}$ for short reach communications," IEEE Photon. Technol. Lett., 27(16), 1757-1760 (2015).

57. M. Morsy-Osman, M. Chagnon, M. Poulin, S. Lessard, and D. V. Plant, "224-Gb/s 10-km Transmission of PDM PAM-4 at $1.3 \mu \mathrm{m}$ Using a Single Intensity-Modulated Laser and a Direct-Detection MIMO DSP-Based Receiver," J. Lightwave Technol. 33, 1417-1424 (2015).

58. M. Morsy-Osman, M. Chagnon, and D. V. Plant, "Four-Dimensional Modulation and Stokes Direct Detection of Polarization Division Multiplexed Intensities, Inter Polarization Phase and Inter Polarization Differential Phase," J. Lightwave Technol. 34, 1585-1592 (2016) .

59. A.Rahim, A. Abbasi, N. Andre, A. Katumba, H. Louchet, K. Van Gasse, R. Baets, G. Morthier, and G. Roelkens. "69 Gb/s DMT direct modulation of a Heterogeneously Integrated InP-on-Si DFB Laser," in Optical Fiber Communication Conference, OSA Technical Digest Series (Optical Society of America, 2017), paper Th1B-5.

60. M. Huang, P. Cai, S. Li, T.-I. Su, L. Wang, W. Chen, C. Hong, and D. Pan. "Cost-effective 25G APD TO-Can/ROSA for 100G applications," in Optical Fiber Communication Conference, OSA Technical Digest Series (Optical Society of America, 2017), Th3B-3.

61. A. Chiuchiarelli, R. Gandhi, S. Rossi, S. Behtash, L. H. Carvalho, F. Caggioni, J. C. Oliveira, and J. Reis. "Single Wavelength 100G Real-Time Transmission for High-Speed Data Center Communications," in Optical Fiber Communication Conference, OSA Technical Digest Series (Optical Society of America, 2017), paper W4I-2.

62. Z. Li, M. S. Erkilinc, K. Shi, E. Sillekens, L. Galdino, B. C. Thomsen, P. Bayvel, and R. Killey. "Performance Improvement of Electronic Dispersion Post-Compensation in Direct 
Detection Systems Using DSP-Based Receiver Linearization," in Optical Fiber Communication Conference, OSA Technical Digest Series (Optical Society of America, 2017), paper Th3D-2.

63. X. Hong, O. Ozolins, C. Guo, X. Pang, J. Zhang, J. R. Navarro, and A. Kakkar "1.55- $\mu$ m EML-based DMT Transmission with Nonlinearity-Aware Time Domain Super-Nyquist Image Induced Aliasing," In Optical Fiber Communication Conference), in Optical Fiber Communication Conference, OSA Technical Digest Series (Optical Society of America, 2017), paper Th3D-3.

64. R. Hirai, N. Kikuchi, and T. Fukui. "High-Spectral Efficiency DWDM transmission of 100Gbit/s/lambda IM/DD Single Sideband-Baseband-Nyquist-PAM8 Signals," in Optical Fiber Communication Conference, OSA Technical Digest Series (Optical Society of America, 2017), paper Th3D-4.

65. K. Hasebe, W. Kobayashi, N. Fujiwara, T. Shindo, T. Yoshimatsu, S. Kanazawa, and T. Ohno, "28-Gbit/s 80-km transmission using SOA-assisted extended-reach EADFB laser (AXEL)," in Optical Fiber Communication Conference, OSA Technical Digest Series (Optical Society of America, 2017), paper Th4G-4.

66. K.P. Zhong, X. Zhou, Y. Wang, J., L. Zeng, C. Yu, A. P. T. Lau, and C. Lu, "Amplifier-Less Transmission of 56Gbit/s PAM4 over 60km Using 25Gbps EML and APD," in Optical Fiber Communication Conference, OSA Technical Digest Series (Optical Society of America, 2017), paper Tu2D-1.

67. Q. Zhang, N. Stojanovic, T. Zuo, L. Zhang, F. Karinou, and E. Zhou, "Single-Lane 180 Gb/s SSB-Duobinary-PAM-4 Signal Transmission over 13 km SSMF," in Optical Fiber Communication Conference, OSA Technical Digest Series (Optical Society of America, 2017), paper Tu2D-2.

68. N. Eiselt, H. Griesser, M. H. Eiselt, W. Kaiser, S. Aramideh, J.J.V. Olmos, I. Tafur Monroy, and J.-P. Elbers, "Real-Time $200 \mathrm{~Gb} / \mathrm{s}(4 \times 56.25 \mathrm{~Gb} / \mathrm{s})$ PAM-4 Transmission over $80 \mathrm{~km}$ SSMF using Quantum-Dot Laser and Silicon Ring-Modulator," in Optical Fiber Communication Conference, OSA Technical Digest Series (Optical Society of America, 2017) paper W4D-3.

69. M. Chagnon, and D. Plant, "504 and 462 Gb/s Direct Detect Transceiver for Single Carrier Short-Reach Data Center Applications," in Optical Fiber Communication Conference, OSA Technical Digest Series (Optical Society of America, 2017), paper W3B-2.

70. R. van der Linden, N.-C. Tran, E. Tangdiongga, and A. Koonen, "Demonstration and Application of $37.5 \mathrm{~Gb} / \mathrm{s}$ Duobinary-PAM3 in PONs," in Optical Fiber Communication Conference, OSA Technical Digest Series (Optical Society of America, 2017), paper Tu3G-4.

71. K.-P. Ho and J. M. Kahn, "Electronic compensation technique to mitigate nonlinear phase noise," Journal of Lightwave Technology, 22(3), 779-783 (2004).

72. E. Lichtman and S. G. Evangelides, "Reduction of the nonlinear impairment in ultralong lightwave systems by tailoring the fibre dispersion," Electronics Letters, 30(4), 346-348 (1994).

73. M. Murakami, T. Takahashi, M. Aoyama, M. Amemiya, M. Sumida, N. Ohkawa, Y. Fukada, T. Imai, and M. Aiki, "2.5 Gbit/s-9720 km, $10 \mathrm{Gbit} / \mathrm{s}-6480 \mathrm{~km}$ transmission in the FSA commercial system with $90 \mathrm{~km}$ spaced optical amplifier repeaters and dispersion-managed cables," Electronics Letters, 31(10), 814-816 (1995).

74. M. Suzuki, I. Morita, N. Edagawa, S. Yamamoto, H. Taga, and S. Akiba, "Reduction of Gordon-Haus timing jitter by periodic dispersion compensation in soliton transmission," Electronics Letters, 31(23), 2027-2029 (1995).

75. A. D. Ellis, J. D. Cox, D. Bird, J. Regnault, J. V. Wright, and W. A. Stallard, "5 Gbit/s soliton propagation over $350 \mathrm{~km}$ with large periodic dispersion coefficient perturbations using erbium doped fibre amplifier repeaters," Electronics Letters, 27(10), 878-880 (1991).

76. J. H. B. Nijhof, N. J. Doran, W. Forysiak, and A. Berntson, "Energy enhancement of dispersion-managed solitons and WDM," Electronics Letters, 34(5), 481-482 (1998).

77. P. Kaewplung, T. Angkaew, and K. Kikuchi, "Complete analysis of sideband instability in chain of periodic dispersion-managed fiber link and its effect on higher order dispersionmanaged long-haul wavelength-division multiplexed systems," Journal of Lightwave Technology, 20(11), 1895-1907 (2002).

78. A. A. Redyuk, O. E. Nanii, V. N. Treshchikov, V. Mikhailov, and M. P. Fedoruk, " 100 Gb s s$^{-1}$ coherent dense wavelength division multiplexing system reach extension beyond the limit of electronic dispersion compensation using optical dispersion management," Laser Physics Letters, 12(2), 025101 (2014).

79. X. Liu, C. Sethumadhavan, and P. J. Winzer, "Dispersion management for inhomogeneous fiber-optic links," US Patent 9160456B2, 2013. 
80. L. Yi, X. Wang, Z. Li, J. Huang, J. Han, and W. Hu, "Upstream dispersion management supporting $100 \mathrm{~km}$ differential reach in TWDM-PON," Opt. Express 23, 7971-7977 (2015)

81. S. H. Cho J-H. Lee, J-H. Lee, E-G. Lee, H. H. Lee, E-S. Jung, and S. S. Lee., "1.25 Gb/s operation of ASE injected RSOA with $50 \mathrm{GHz}$ channel spacing by using injection current adjustment, dispersion management and receiver with decision threshold level control," In Proceedings of the $12^{\text {th }}$ International Conference on Transparent Optical Networks, (IEEE, 2010), paper Tu.B1.6.

82. J. P. Gordon and H. A. Haus, "Random walk of coherently amplified solitons in optical fiber transmission," Opt. Lett. 11, 665-667 (1986).

83. D. Wood, "Constraints on the bit rates in direct detection optical communication systems using linear or soliton pulses," Journal of Lightwave Technology, 8(7), 1097-1106 (1990).

84. E.M. Dianov, A.V. Luchnikov, A.N. Pilipetskii, and A.M. Prokhorov, "Long-range interaction of picosecond solitons through excitation of acoustic waves in optical fibers," Appl. Phys. B 54(2), 175-180 (1992).

85. J.M. Jacob, E.A. Golovchenko, A.N. Pilipetskii, G.M. Carter, and C.R. Menyuk, "10-Gb/s transmission of NRZ over $10000 \mathrm{~km}$ and solitons over $13500 \mathrm{~km}$ error-free in the same dispersion-managed system," IEEE Photonics Technology Letters, 9(10), 1412-1414 (1997).

86. Yuji Kodama and Akira Hasegawa, "Generation of asymptotically stable optical solitons and suppression of the Gordon-Haus effect," Opt. Lett. 17, 31-33 (1992).

87. N. J. Smith, K. J. Blow, K. Smith, and W. J. Firth, "Suppression of soliton interactions by periodic phase modulation," Opt. Lett. 19, 16-18 (1994)

88. W. Forysiak and N. J. Doran, "Reduction of Gordon-Haus jitter in soliton transmission systems by optical phase conjugation," Journal of Lightwave Technology, 13(5), 850-855 (1995).

89. M. Nakazawa, E. Yamada, H. Kubota, and K. Suzuki, "10 Gbit/s soliton data transmission over one million kilometres," Electronics Letters, 27(14), 1270-1272 (1991).

90. N. J. Doran, "Soliton communications systems: the concept is alive," in Proceedings of 14th Annual Meeting of the IEEE Lasers and Electro-Optics Society (IEEE, 2001), pp. 214-215.

91. O. Yushko, A. Redyuk, M. Fedoruk, K. J. Blow, N. J. Doran, A. D. Ellis, and S. Turitsyn, "Timing and phase jitter suppression in coherent soliton transmission," Opt. Lett. 39, 6308$6311(2014)$

92. S.E. Alavi, I.S. Amiri, S.M. Idrus, A.S. Supa'at, J. Ali J, and P.P. Yupapin, "All-optical OFDM generation for IEEE802. 11a based on soliton carriers using microring resonators," IEEE Photonics Journal 6(1), 1-9 (2014).

93. R. Sharma and G. Garg; "Path Averaged Soliton Systems for Long-Haul Communication," International Journal of Research and Engineering, 4(1), 22-27 (2017).

94. R. Nagesh, R. Mohan, and R.S. Asha, "A Survey on Dispersion Management Using Optical Solitons in Optical Communication System," Procedia Technology, 25, 552-559 (2016).

95. W. Liu, Y. Zhang, L. Pang, H. Yan, G. Ma, and M. Lei, "Study on the control technology of optical solitons in optical fibers," Nonlinear Dynamics, 86(2), 1069-1073 (2016).

96. S. Hari, M. I. Yousefi, and F. R. Kschischang, "Multieigenvalue Communication," J. Lightwave Technol. 34, 3110-3117 (2016)

97. S.A. Derevyanko, J. E. Prilepsky, and S. K. Turitsyn. "Capacity estimates for optical transmission based on the nonlinear Fourier transform," Nature Communications, 7, 12710 (2016).

98. S. Sugimoto, K. Minemura; K. Kobayashi; M. Seki; M. Shikada; A. Ueki; T. Yanase; T. Miki, "High-speed digital-signal transmission experiments by optical wavelength-division multiplexing," Electronics Letters, 13(22), 680-682 (1977).

99. P. J. Winzer, "Scaling Optical Fiber Networks: Challenges and Solutions," Optics \& Photonics News 26(3), 28-35 (2015).

100. C. E. Shannon, “A mathematical theory of communication,” Bell Syst. Tech. J., 27, 379-423, 623-656, (1948).

101. K.O. Hill, D. C. Johnson, B. S. Kawasaki, and R. I. MacDonald. "cw three-wave mixing in single-mode optical fibers," Journal of Applied Physics 49(10), 5098-5106 (1978).

102. A. D. Ellis and W. A. Stallard, "Four wave mixing in ultra long transmission systems incorporating linear amplifiers," in Proceedings of the IEE Colloquium on Non-Linear Effects in Fibre Communications, (IEE, 1990), 6/1-6/4.

103. D. G. Schadt, "Effect of amplifier spacing on four-wave mixing in multichannel coherent communications," in Electronics Letters, 27(20), 1805-1807 (1991).

104. K. Inoue, "Phase-mismatching characteristic of four-wave mixing in fiber lines with multistage optical amplifiers," Opt. Lett. 17, 801-803 (1992). 
105. N. Shibata, R. Braun, and R. Waarts, "Phase-mismatch dependence of efficiency of wave generation through four-wave mixing in a single-mode optical fiber," IEEE Journal of Quantum Electronics, 23(7), 1205-1210 (1987).

106. D.A.Cleland, X.Gu, A.D.Ellis, and J.D.Cox, "Limitations of WDM transmission over $560 \mathrm{~km}$ due to degenerate four wave mixing," Electronics Letters, 28(3), 307-308 (1992).

107. C. Kurtzke, "Suppression of fiber nonlinearities by appropriate dispersion management," IEEE Photonics Technology Letters, 5(10), 1250-1253 (1993).

108. W. Zeiler, F. Di Pasquale, P. Bayvel, and J. E. Midwinter, "Modeling of four-wave mixing and gain peaking in amplified WDM optical communication systems and networks," Journal of Lightwave Technology, 14(9), 1933-1942 (1996).

109. K. Inoue and H. Toba, "Fiber four-wave mixing in multi-amplifier systems with nonuniform chromatic dispersion," in Journal of Lightwave Technology, 13(1), 88-93 (1995).

110. M. E. Marhic, N. Kagi, T. K. Chiang, and L. G. Kazovsky, "Optimizing the location of dispersion compensators in periodically amplified fiber links in the presence of third-order nonlinear effects," IEEE Photonics Technology Letters, 8(1), 145-147 (1996).

111. K. Nakajima, M. Ohashi, K. Shiraki, T. Horiguchi, K. Kurokawa, and Y. Miyajima, "Fourwave mixing suppression effect of dispersion distributed fibers," Journal of Lightwave Technology, 17(10), 1814-1822 (1999).

112. M. Manna and E. A. Golovchenko, "FWM resonances in dispersion slope-matched and nonzero-dispersion fiber maps," in IEEE Photonics Technology Letters, 14(7), 929-931 (2002).

113. E. A. Golovchenko, N. S. Bergano and C. R. Davidson, "Four-wave mixing in multispan dispersion-managed transmission links," IEEE Photonics Technology Letters, 10(10), 14811483 (1998).

114. M.A.Z.Al Khateeb, M. Tan, M.A. Iqbal, M. McCarthy, P. Harper and A.D. Ellis, "Four Wave Mixing in Distributed Raman Amplified Optical Transmission Systems" in Proceedings of IEEE Photonics Conference, (IEEE 2016), paper Th.B1.1

115. V. Pechenkin and I. J. Fair, "On Four-Wave Mixing Suppression in Dispersion-Managed Fiber-Optic OFDM Systems With an Optical Phase Conjugation Module," Journal of Lightwave Technology, 29(11), 1678-1691 (2011).

116. R. I. Killey, H. J. Thiele, V. Mikhailov, and P. Bayvel, "Prediction of transmission penalties due to cross-phase modulation in WDM systems using a simplified technique," IEEE Photonics Technology Letters, 12(7), 804-806 (2000).

117. J. M. Kahn and Keang-Po Ho, "Spectral efficiency limits and modulation/detection techniques for DWDM systems," IEEE Journal of Selected Topics in Quantum Electronics, 10(2), 259272 (2004).

118. A.Splett, C. Kurtzke, and K. Petermann. "Ultimate Transmission Capacity of Amplified Optical Fiber Communication Systems taking into Account Fiber Nonlinearities", in Proceedings of European Conference and Exhibition on Optical Communication (EPF, 1993), paper MoC2.4.

119. K.J. Blow, N. J. Doran, and J. R. Taylor, "Nonlinear propagation effects in optical fibers: numerical studies," in Optical Solitons-Theory and Experiment, J.R.Taylor, ed, (Cambridge University Press, 1992), pp. 73-106.

120. O. V. Sinkin, R. Holzlöhner, J. Zweck, and C. R. Menyuk, "Optimization of the Split-Step Fourier Method in Modeling Optical-Fiber Communications Systems," J. Lightwave Technol. 21(1), 61-68 (2003).

121. L. G. Kazovsky, "Phase- and polarization-diversity coherent optical techniques," Journal of Lightwave Technology, 7(2), 279-292 (1989).

122. P. M. Hill, R. Olshansky and W. K. Burns, "Optical polarization division multiplexing at 4 Gb/s," in IEEE Photonics Technology Letters, 4(5), 500-502, May 1992.

123. S. Tsukamoto, D. S. Ly-Gagnon, K. Katoh, and K. Kikuchi, "Coherent demodulation of 40Gbit/s polarization-multiplexed QPSK signals with 16-GHz spacing after 200-km transmission," in Optical Fiber Communication Conference, OSA Technical Digest Series (Optical Society of America, 2005), paper PDP29.

124. G. R. Walker, D. M. Spirit, P. J. Chidgey, E. G. Bryant, and C. R. Batchellor, "Effect of fibre dispersion on four-wave mixing in multichannel coherent optical transmission system," Electronics Letters, 28(11), 989-991 (1992).

125. S. Watanabe, T. Terahara, I. Yokota, T. Naito, T. Chikama, and H. Kuwahara, "Optical coherent broad-band transmission for long-haul and distribution systems using subcarrier multiplexing," Journal of Lightwave Technology, 11(1), 116-127 (1993).

126. S.J. Savory, "Digital filters for coherent optical receivers," Optics Express 16(2), 804-817 (2008). 
127. K. Roberts, S.H. Foo, M. Moyer, M. Hubbard, A. Sinclair, J. Gaudette, and C. Laperle, "High Capacity Transport—100G and Beyond," Journal of Lightwave Technology, 33(3), 563-578 (2015).

128. M. Nakazawa, K. Kikuchi, and T. Miyazaki, High Spectral Density Optical Communication Technologies, (Springer, 2010).

129. S. Yamashita and T. Okoshi, "Suppression of beat noise from optical amplifiers using coherent receivers," Journal of Lightwave Technology, 12(6), 1029-1035 (1994).

130. F. Derr, "Coherent optical QPSK intradyne system: concept and digital receiver realization," Journal of Lightwave Technology, 10(9), 1290-1296 (1992).

131. H. Nyquist, "Certain topics in telegraph transmission theory", Proc. AIEE Trans., 47(2), $617-$ 644 (1928).

132. D. O. North, "The Absolute Sensitivity of Radio Receivers," RCA Review, 6(3), 332-43 (1942)

133. A. Lender, "Correlative level coding for binary-data transmission", IEEE Spectrum, 3(2), 104-115 (1966).

134. F.Fresi, M. Secondini, G. Berrettini, G.Meloni, and L.Poti, "Impact of optical and electrical narrowband spectral shaping in faster than Nyquist Tb superchannel”, Photonics Technology Letters, 25(23), 2301-2304 (2013).

135. V.Arya, I. Jacobs, “Optical preamplifier receiver for spectrum sliced WDM," Journal of Lightwave Technology, 15(4), 576-583 (1997).

136. M. Sorokina, S. Sygletos, and S.K. Turitsyn , "Shannon Capacity of Nonlinear Communication Channels" in Conference on Lasers and Electrooptics, (Optical Society of America, 2016), paper SM3F4.

137. R. R. Mosier and R. G. Clabaugh, "Kineplex, a bandwidth-efficient binary transmission system," Transactions of the American Institute of Electrical Engineers, Part I: Communication and Electronics, 76(6), 723-728 (1958).

138. H. W. Chang, "Synthesis of band-limited orthogonal signals for multichannel data transmission," The Bell System Technical Journal, 45(10), 1775-1796 (1966).

139. B. Farhang-Boroujeny, "OFDM Versus Filter Bank Multicarrier," IEEE Signal Processing Magazine, 28(3), 92-112 (2011).

140. G. Bosco, A. Carena, V. Curri, P. Poggiolini, and F. Forghieri, "Performance Limits of Nyquist-WDM and CO-OFDM in High-Speed PM-QPSK Systems," IEEE Photonics Technology Letters, 22(15), 1129-1131 (2010).

141. M Sorokina, S Sygletos, and S Turitsyn, "Ripple distribution for nonlinear fibre-optic channels," Optics Express, 25, 2228-2238 (2017).

142. J. Tang, "The channel capacity of a multispan DWDM system employing dispersive nonlinear optical fibers and an ideal coherent optical receiver," Journal of Lightwave Technology, 20(7), 1095-1101 (2002).

143. P. P. Mitra and J. B. Stark, "Nonlinear limits to the information capacity of optical fibre communications," Nature 411, 1027-1030 (2001).

144. K. .S. Turitsyn, S.A. Derevyanko, I.V. Yurkevich, and S. K. Turitsyn, "Information capacity of optical fiber channels with zero average dispersion," Phys. Rev. Letters, 91, 203901-203904 (2003).

145. X.Chen and W. Shieh, "Closed-form expressions for nonlinear transmission performance of densely spaced coherent optical OFDM systems," Opt. Express, 18(18), 19039-54 (2010).

146. H. Louchet, A. Hodzic, and K. Petermann, "Analytical model for the performance evaluation of DWDM transmission systems," IEEE Photonics Technology Letters, 15(9), 1219-1221 (2003).

147. K. Igarashi, T. Tsuritani, I. Morita, Y. Tsuchida, K. Maeda, M. Tadakuma, and M. Suzuki, "Super-Nyquist-WDM transmission over 7,326-km seven-core fiber with capacity-distance product of 1.03 Exabit/s· km," Optics Express, 22(2), 1220-1228 (2014).

148. T. Kan, K. Kasai, M. Yoshida, and M. Nakazawa, "42.3-Tbit/s, 18-Gbaud 64QAM WDM Coherent Transmission of $160 \mathrm{~km}$ over Full C-band using an Injection Locking Technique with a Spectral Efficiency of $9 \mathrm{bit} / \mathrm{s} / \mathrm{Hz}$," in Optical Fiber Communication Conference, OSA Technical Digest Series (Optical Society of America, 2017), paper Th3F5

149. D. Hillerkuss, R. Schmogrow, T. Schellinger, M. Jordan, M. Winter, G. Huber, T. Vallaitis, R. Bonk, P. Kleinow, F. Frey, M. Roeger, S. Koenig, A. Ludwig, A. Marculescu, J. Li, M. Hoh, M. Dreschmann, J. Meyer, S. Ben Ezra, N. Narkiss, B. Nebendahl, F. Parmigiani, P. Petropoulos, B. Resan, A. Oehler, K. Weingarten, T. Ellermeyer, J. Lutz, M. Moeller, M. Huebner, J. Becker, C. Koos, W. Freude, and J. Leuthold, "26 Tbit s-1 line-rate super-channel transmission utilizing all-optical fast Fourier transform processing," Nature Photonics 5(6), 364-371 (2011). 
150. S. Chandrasekhar, X. Liu, B. Zhu, and D. W. Peckham. "Transmission of a 1.2-Tb/s 24-carrier no-guard-interval coherent OFDM superchannel over 7200-km of ultra-large-area fiber." In Proceedings of $35^{\text {th }}$ European Conference and Exhibition of Optical Communication (IEEE, 2009).

151. G. Gao, X. Chen, and W. Shieh, "Influence of PMD on fiber nonlinearity compensation using digital back propagation," Opt. Express 20, 14406-14418 (2012).

152. M. A. Z. Al-Khateeb, M.E. McCarthy, C.S. Costa, and A.D. Ellis, "Effect of second order signal-noise interactions in nonlinearity compensated optical transmission systems" Opt. Lett. 41, 1849-1852 (2016)

153. D. Rafique and A. D. Ellis, "Impact of signal-ASE four-wave mixing on the effectiveness of digital back-propagation in $112 \mathrm{~Gb} / \mathrm{s}$ PM-QPSK systems," Opt. express, 19(4), 3449-3454 (2011)

154. P. Serena, "Nonlinear Signal-Noise Interaction in Optical Links with Nonlinear Equalization," Journal of Lightwave technology, 34(6), 1476-1483 (2016).

155. J. P. Gordon and L. F. Mollenauer, "Phase noise in photonic communications systems using linear amplifiers," Opt. Lett. 15, 1351-1353 (1990).

156. P. Poggiolini, A. Carena, Y. Jiang, G. Bosco, V. Curri and F. Forghieri, "Impact of low-OSNR operation on the performance of advanced coherent optical transmission systems," in Proceedings of The European Conference on Optical Communication (IEEE, 2014), paper Mo4.3.2

157. G. Gao, X. Chen, and W. Shieh, "Analytical Expressions for Nonlinear Transmission Performance of Coherent Optical OFDM Systems With Frequency Guard Band”, Journal of Lightwave Technology, 30(15), 2447-2454 (2012).

158. A. D. Ellis, M. Tan, M. A. Iqbal, M. A. Z. Al Khateeb, V. Gordienko, G. Saavedra. M., S. Fabbri, M. F. C. Stephens, M. E. McCarthy, A. Perentos, I. D. Phillips, D. Lavery, G. Liga, R. Maher, P. Harper, N. J. Doran, S. K. Turitsyn, S. Sygletos, and P. Bayvel, "4 Tb/s Transmission Reach Enhancement Using $10 \times 400 \mathrm{~Gb} / \mathrm{s}$ Super-Channels and Polarization Insensitive Dual Band Optical Phase Conjugation," Journal of Lightwave Technology, 34(8), 1717-1723 (2016).

159. F. Vacondio, O. Rival, C. Simonneau, E. Grellier, A. Bononi, L. Lorcy, J.-C. Antona, and S. Bigo, "On nonlinear distortions of highly dispersive optical coherent systems," Opt. Express 20(2), 1022-1032 (2012)

160. D. Rafique and A.D. Ellis, "Digital back-propagation for spectrally efficient WDM 112 Gbit/s PM m-ary QAM transmission," Optics Express, 19(6), 5219-5224 (2011).

161. A.D. Ellis, S. T. Le, M. A. Z. Al-Khateeb, S. K. Turitsyn, G. Liga, D. Lavery, T. Xu, and P. Bayvel, "The Impact of Phase Conjugation on the Nonlinear-Shannon Limit", In Proceedings of the 2015 IEEE Summer Topicals Meeting Series (IEEE, 2015), 209-210.

162. P. Poggiolini, "The GN Model of Non-Linear Propagation in Uncompensated Coherent Optical Systems," Journal of Lightwave Technology, 30(24), 3857-3879 (2012).

163. H. Kim and A. H. Gnauck, "Experimental investigation of the performance limitation of DPSK systems due to nonlinear phase noise," IEEE Photonics Technology Letters, 15(2), 320 322 (2003).

164. R. Dar, M. Shtaif, and M. Feder, "New bounds on the capacity of the nonlinear fiber-optic channel," in Opt. Lett., 39, 398-401 (2014).

165. C-Y Lin, R. Asif, M. Holtmannspoetter, and B. Schmauss, "Nonlinear mitigation using carrier phase estimation and digital backward propagation in coherent QAM transmission," Opt. Express 20, B405-B412 (2012)

166. R. J. Essiambre, G. Kramer, P. J. Winzer, G. J. Foschini, and B. Goebel, "Capacity Limits of Optical Fiber Networks," Journal of Lightwave Technology, 28(4), 662-701 (2010).

167. N.J.Doran and A.D.Ellis, "Minimising total energy requirements in amplified links by optimising amplifier spacing," Optics Express, 22(16), 19810-19817 (2014).

168. Y. Sun, O. V. Sinkin, A. V. Turukhin, M. A. Bolshtyansky, D. G. Foursa, and A. N. Pilipetskii, "SDM for Power Efficient Transmission", in Optical Fiber Communication Conference, OSA Technical Digest Series (Optical Society of America, 2017), paper M2F1.

169. J. X. Cai, Y. Sun, H. Zhang, H. G. Batshon, M. V. Mazurczyk, O. V. Sinkin, D. G. Foursa, and A. Pilipetskii "49.3 Tb/s Transmission Over $9100 \mathrm{~km}$ Using C+L EDFA and $54 \mathrm{~Tb} / \mathrm{s}$ Transmission Over 9150 km Using Hybrid-Raman EDFA," Journal of Lightwave Technology, 33(13), 2724-2734 (2015).

170. A. Nespola, S. Straullu, A. Carena, G. Bosco, R. Cigliutti, V. Curri, and J. Bauwelinck, "GNModel Validation Over Seven Fiber Types in Uncompensated PM-16QAM Nyquist-WDM Links," IEEE Photonics Technology Letters, 26(2), 206-209 (2014). 
171. J. Stark, Y. T. Hsueh, T. F. Detwiler, M. M. Filer, S. Tibuleac, and S. E. Ralph, "System Performance Prediction With the Gaussian Noise Model in 100G PDM-QPSK Coherent Optical Networks," Journal of Lightwave Technology, 31(21), 3352-3360 (2013).

172. R.I. Killey, R. Maher, T. Xu, L. Galdino, M. Sato, S. Kilmurray, and P. Bayvel, "Experimental characterisation of digital Nyquist pulse-shaped dual-polarisation 16QAM WDM transmission and comparison with the Gaussian noise model of nonlinear propagation," In Proceedings of International Conference on Transparent Optical Networks, (IEEE, 2014), paper TuD1.3.

173. N. Rossi, P. Ramantanis, and J. C. Antona, "Nonlinear interference noise statistics in unmanaged coherent networks with channels propagating over different lightpaths," in Proceedings of $40^{\text {th }}$ European Conference and Exhibition of Optical Communication (IEEE, 2014), paper Mo4.3.4.

174. O. Golani, M. Feder, A. Mecozzi, and M. Shtaif, "Correlations and phase noise in NLINmodelling and system implications," in Optical Fiber Communication Conference, OSA Technical Digest (Optical Society of America, 2016), paper W3I2.

175. P. Jennevé, P. Ramantanis, J.C. Antona, G. de Valicourt, M.A. Mestre, H. Mardoyan, and S. Bigo, "Pitfalls of error estimation from measured non-Gaussian nonlinear noise statistics over dispersion-unmanaged systems," in Proceedings of $40^{\text {th }}$ European Conference and Exhibition of Optical Communication (IEEE, 2014), paper Mo4.3.3

176. M. P. Yankov "Experimental Study of Nonlinear Phase Noise and its Impact on WDM Systems with DP-256QAM," in Proceedings of $42^{\text {nd }}$ European Conference and Exhibition of Optical Communication (VDE, 2016), 479-481

177. L. Li, Z. Tao, L. Dou, W. Yan, S. Oda, T. Tanimura, and J.C. Rasmussen, "Implementation efficient nonlinear equalizer based on correlated digital backpropagation," in Optical Fiber Communication Conference, OSA Technical Digest Series (Optical Society of America, 2011), paper OWW3.

178. M. H. Taghavi, G. C. Papen, and P. H. Siegel, "On the Multiuser Capacity of WDM in a Nonlinear Optical Fiber: Coherent Communication,” IEEE Transactions on Information Theory, 52(11), 5008-5022 (2006).

179. E. E. Narimanov and P. Mitra, "The channel capacity of a fiber optics communication system: perturbation theory," J. Lightwave Technol., 20,530-537 (2002).

180. E. Agrell, A. Alvarado, and F. R. Kschischang. "Implications of information theory in optical fibre communications," Phil. Trans. R. Soc. A, 374(2062), 2016

181. M. Secondini, E. Forestieri, and C. R. Menyuk "A combined regular-logarithmic perturbation method for signal-noise interaction in amplified optical systems", J. Lightwave Technol. 27(16), 3358 - 3369 (2009).

182. P. Johannisson and M. Karlsson, "Perturbation analysis of nonlinear propagation in a strongly dispersive optical communication system," J. Lightw. Technol., 31(8), 1273-1282 (2013).

183. M. Secondini, E. Forestieri, and G. Prati, "Achievable information rate in nonlinear WDM fiber-optic systems with arbitrary modulation formats and dispersion maps," J. Lightwave Technol. 31(23), 3839-3852 (2013).

184. E. Agrell, A. Alvarado, G. Durisi, and M. Karlsson, "Capacity of a nonlinear optical channel with finite memory," J.Lightwave Technol. 32(16), 2862-2876 (2014).

185. R. Dar, M. Feder, A. Mecozzi, and M. Shtaif, "Inter-channel nonlinear interference noise in WDM systems: modeling and mitigation," J. Lightwave Technol. 33(5), 1044-1053 (2015).

186. M. Secondini; E. Forestieri, "Scope and Limitations of the Nonlinear Shannon Limit," Journal of Lightwave Technology, 35(4), 893-902 (2017).

187. A. D. Ellis, M. E. McCarthy, M. A. Z. Al-Khateeb, and S. Sygletos, "Capacity limits of systems employing multiple optical phase conjugators," Opt. Express, 23, 20381-20393 (2015).

188. M. Nazarathy, J. Khurgin, R. Weidenfeld, Y. Meiman, P. Cho, R. Noe, I. Shpantzer, and V. Karagodsky, "Phased-array cancellation of nonlinear FWM in coherent OFDM dispersive multi-span links," Opt. Express 16, 15777-15810 (2008).

189. A.D.Ellis and M.E. McCarthy, "Impact of Optical Phase Conjugation on the Shannon Capacity Limit," in Optical Fiber Communication Conference, OSA Technical Digest Series (Optical Society of America, 2016), paper Th4F-2.

190. D. J. Elson, L. Galdino, R. Maher, R. I. Killey, B. C. Thomsen, and P. Bayvel, "High spectral density transmission emulation using amplified spontaneous emission noise," Opt. Lett. 41, 68-71 (2016).

191. N. MacSuibhne, M.E. McCarthy, S.T. Le, S. Sygletos, F.M. Ferreira, and A.D. Ellis, "Optical fibre limits: an approach using ASE channel estimation", in Proceedings of Progress in Electromagnetics Research Symposium, (The Electromagnetics Academy, 2016), 489.. 
192. .M. Sorokina, S. Sygletos, and S. Turitsyn, "Sparse identification for nonlinear optical communication systems: SINO method," Opt. Express 24, 30433-30443 (2016)

193. J. Gonçalves, C. S. Martins, F. P. Guiomar, T. R. Cunha, J. C. Pedro, A. N. Pinto, and P. M. Lavrador, "Nonlinear compensation with DBP aided by a memory polynomial," Opt. Express 24, 30309-30316 (2016)

194. J Thrane, J Wass, M Piels, JCM Diniz, R Jones, and D Zibar, "Machine Learning Techniques for Optical Performance Monitoring from Directly Detected PDM-QAM Signals," Journal of Lightwave Technology 35(4), 868-875 (2016)

195. A. Lord, A. Soppera, and A. Jacquet, "The impact of capacity growth in national telecommunications networks," Phil. Trans. R. Soc. A, 374(2062), 20140431 (2016)

196. Cisco, V. N. I. "Cisco Visual Networking Index: Forecast and Methodology, 2014-2019 White Paper." (2015).

197. O. Yushko, A. Redyuk, M. Fedoruk, K. J. Blow, N. J. Doran, A. D. Ellis, and S. Turitsyn, "Timing and phase jitter suppression in coherent soliton transmission", Optics Letters, 39(21), 6308-6311 (2014).

198. C. Paré, N.J. Doran, A. Villeneuve, and P.A. Bélanger, "Compensating for dispersion and the nonlinear Kerr effect without phase conjugation," Optics letters, 21(7), 459-461 (1996).

199. X. Li, X. Chen, G. Goldfarb, E. Mateo, I. Kim, F. Yaman, and G. Li, "Electronic postcompensation of WDM transmission impairments using coherent detection and digital signal processing," Opt. Express, 2, 881- 888 (2008)

200. L. B. Du, D. Rafique, A. Napoli, B. Spinnler, A. D. Ellis, M.Kuschnerov, and A.J. Lowery, "Digital Fiber Nonlinearity Compensation: Toward 1-Tb/s transport," IEEE Signal Processing Magazine, 31(2), 46-56 (2014).

201. D. McGhan, C. Laperle, A. Savehenko, Chuandong Li, G. Mak and M. O'Sullivan, "5120 km RZ-DPSK transmission over G652 fiber at $10 \mathrm{~Gb} / \mathrm{s}$ with no optical dispersion compensation," in Optical Fiber Communication Conference, OSA Technical Digest Series (Optical Society of America, 2005), paper PDP27.

202. M. G. Taylor, "Coherent detection method using DSP for demodulation of signal and subsequent equalization of propagation impairments," IEEE Photonics Technology Letters, 16(2), 674-676 (2004).

203. N. K. Fontaine, X. Liu, S. Chandrasekhar, R. Ryf, S.Randel, P. Winzer, R.Delbue, P. Pupalaikis, A. Sureka"Fiber nonlinearity compensation by digital backpropagation of an entire 1.2- $\mathrm{Tb} / \mathrm{s}$ superchannel using a full-field spectrally-sliced receiver," In Proceedings of the $39^{\text {th }}$ European Conference and Exhibition on Optical Communication, (IET, 2013), paper Mo.3.D.5

204. E. Temprana, N. Alic, B. P. P. Kuo, and S. Radic," Beating the Nonlinear Capacity Limit" Optics \& Photonics News, 27(3), 30-37 (2016).

205. E. Temprana, E. Myslivets, L. Liu, V. Ataie, A. Wiberg, B.P.P. Kuo, N. Alic, and S. Radic, "Two-fold transmission reach enhancement enabled by transmitter-side digital backpropagation and optical frequency comb-derived information carriers," Opt. Express 23, 20774-20783 (2015).

206. G. Liga, T. Xu, A. Alvarado, R.I. Killey, and P. Bayvel, "On the performance of multichannel digital backpropagation in high-capacity long-haul optical transmission," Opt. Express, 22(24), 30053-30062 (2014).

207. R. Dar and P. J. Winzer, "On the Limits of Digital Back-Propagation in Fully Loaded WDM Systems," IEEE Photonics Technology Letters, 28(11), 1253-1256 (2016).

208. D.M.Pepper, A. Yariv, "Compensation for phase distortions in nonlinear media by phase conjugation", Opt. Lett, 5(2), 59-60 (1980).

209. R.A.Fisher, B.R. Suydam, and D. Yevick, "Optical phase conjugation for time-domain undoing of dispersion self-phase modulation effect" Opt. Lett., 8(12), 611-613 (1983).

210. A. H. Gnauck, R. M. Jopson, P. P. Iannone, and R. M. Derosier, "Transmission of two wavelength-multiplexed $10 \mathrm{Gbit} / \mathrm{s}$ channels over $560 \mathrm{~km}$ of dispersive fibre," Electronics Letters, 30(9), 727-728 (1994).

211. S. Watanabe and M. Shirasaki, "Exact compensation for both chromatic dispersion and Kerr effect in a transmission fiber using optical phase conjugation," Journal of Lightwave Technology, 14(3), 243-248 (1996).

212. D. D. Marcenac, D. Nesset, A.E. Kelly, M. Brierly, A.D. Ellis, D.G. Moodie, and C.W. Ford, "40 Gbit/s transmission over $406 \mathrm{~km}$ of NDSF using mid-span spectral inversion by fourwave-mixing in a $2 \mathrm{~mm}$ long semiconductor optical amplifier," Electronics Letters, 33(10), 879-880 (1997).

213. A. D. Ellis, M.A.Z. Al Khateeb, and M.E.McCarthy, "Impact of optical phase conjugation on the nonlinear Shannon limit", Journal of Lightwave Technology, 35(4), 792-798 (2017). 
214. S.T. Le, M.E. McCarthy, S. K. Turitsyn, I. Phillips, G. Liga, D. Lavery, T.Xu, P. Bayvel, and A.D. Ellis, "Optical and Digital Phase Conjugation Techniques for Fiber Nonlinearity Compensation", in Proceedings of 2015 Opto-Electronics and Communications Conference (OECC), (IEEE, 2015), paper 7340113.

215. D. Rafique, S. Sygletos, and A. D. Ellis, "Intra-channel nonlinearity compensation for PM16QAM traffic co-propagating with 28Gbaud m-ary QAM neighbours," Optics Express, 21, 4174-4182 (2013).

216. D. Lavery, D. Ives, G. Liga, A. Alvarado, S. J. Savory, and P. Bayvel, "The Benefit of Split Nonlinearity Compensation for Single-Channel Optical Fiber Communications," IEEE Photonics Technology Letters, 28(17), 1803-1806 (2016).

217. T. Tanimura, T. Kato, R. Okabe, S. Oda, T. Richter, R. Elschner, C. Schmidt-Langhorst, C. Schubert, J. Rasmussen, and S. Watanabe, "Coherent Reception and 126 GHz Bandwidth Digital Signal Processing of CO-OFDM Superchannel Generated By Fiber Frequency Conversion," in Optical Fiber Communication Conference, OSA Technical Digest Series (Optical Society of America, 2014), paper Tu3A.1.

218. M. Mussolin, D. Rafique, J. Mårtensson, M. Forzati, J. K. Fischer, L. Molle, M. Nölle, C. Schubert, and A. Ellis, "Polarization Multiplexed $224 \mathrm{~Gb} / \mathrm{s}$ 16QAM Transmission Employing Digital Back-Propagation," in Proceedings of 37th European Conference and Exposition on Optical Communications, OSA Technical Digest Series (Optical Society of America, 2011), paper We.8.B.6.

219. I. Sackey, F. Da Ros, J.K. Fischer, T. Richter, M. Jazayerifar, C. Peucheret, K. Petermann, and Colja Schubert, "Kerr Nonlinearity Mitigation: Mid-Link Spectral Inversion Versus Digital Backpropagation in 5×28-GBd PDM 16-QAM Signal Transmission," J. Lightwave Technol. 33, 1821-1827 (2015).

220. E. P. Silva, M. P. Yankov, F. Da Ros, and S. Forchhammer, "Experimental Comparison of Gains in Achievable Information Rates from Probabilistic Shaping and Digital Backpropagation for DP-256QAM / 1024QAM WDM Systems," in Proceedings of the 42nd European Conference and Exhibition on Optical Communication (VDE, 2016), 43-45.

221. F. Zhang, Q. Zhuge, M. Qiu, W. Wang, M. Chagnon, and D. V. Plant, "XPM Model-Based Digital Backpropagation for Subcarrier-Multiplexing Systems," J. Lightwave Technol. 33, 5140-5150 (2015)

222. R. Maher, L. Galdino, M. Sato, T. Xu, K. Shi, S. Kilmurray, S. J. Savory, B. C. Thomsen, R. I. Killey, and P. Bayvel, "Linear and nonlinear impairment mitigation in a Nyquist spaced DP16QAM WDM transmission system with full-field DBP," in Proceedings of The European Conference on Optical Communication (IEEE, 2014), paper P.5.10.

223. T. Omiya, M. Yoshida, and M. Nakazawa, "400 Gbit/s 256 QAM-OFDM transmission over $720 \mathrm{~km}$ with a $14 \mathrm{bit} / \mathrm{s} / \mathrm{Hz}$ spectral efficiency by using high-resolution FDE," Opt. Express 21, 2632-2641 (2013).

224. C. Xia, X. Liu, S. Chandrasekhar, N. K. Fontaine, Likai Zhu, and G. Li, "Multi-channel nonlinearity compensation of PDM-QPSK signals in dispersion-managed transmission using dispersion-folded digital backward propagation," Opt. Express 22, 5859-5866 (2014).

225. D. Rafique, M. Mussolin, M. Forzati, J. Martensson, M. Chugtai, and A.D. Ellis, "Compensation of intra channel nonlinear fibre impairments using simplified digital back propagation algorithm," Opt. Express, 19(10),9453-9460 (2011).

226. L. Liu, L. Li, Y. Huang, K. Cui, Q. Xiong, F. N. Hauske, C. Xie, abd Y. Cai, "Intrachannel Nonlinearity Compensation by Inverse Volterra Series Transfer Function," Journal of Lightwave Technology, 30(3), 310-316 (2012).

227. S. T. Le, M. E. McCarthy, N. M. Suibhne, A. D. Ellis, and S. K. Turitsyn, "Phase-Conjugated Pilots for Fibre Nonlinearity Compensation in CO-OFDM Transmission," Journal of Lightwave Technology, 33(7), 1308-1314 (2015).

228. B. Inan, S. Randel, S. L. Jansen, A. Lobato, S. Adhikari, and N. Hanik, "Pilot-tone-based nonlinearity compensation for optical OFDM systems," In Proceedings 36th European Conference and Exhibition on Optical Communication, (IEEE, 2010), paper Tu4A6.

229. R.I.Killey, P. M. Watts, V. Mikhailov, M. Glick, and P. Bayvel. "Electronic dispersion compensation by signal predistortion using digital processing and a dual-drive Mach-Zehnder modulator," IEEE Photonics Technology Letters 17(3), 714-716 (2005).

230. J. C. Cartledge, A. D. Ellis, A. Shiner, A. I. A. El-Rahman, M.E. McCarthy, M. Reimer, A. Borowiec, and A. Kashi, "Signal Processing Techniques for Reducing the Impact of Fiber Nonlinearities on System Performance", in Optical Fiber Communication Conference, OSA Technical Digest (Optical Society of America, 2016), paper Th4F.5.

231. R. Maher, D. Lavery, D. Millar, A Alvarado, K. Parsons, R. Killey, and P. Bayvel, "Reach enhancement of $100 \%$ for a DP-64QAM super-channel using MC-DBP," in Optical Fiber 
Communication Conference, OSA Technical Digest Series (Optical Society of America, 2015), paper Th4D5.

232. M. P. Yankov, F. Da Ros, E. P. Silva, T. Fehenberger, L. Barletta, D. Zibar, L. K. Oxenløwe, M. Galili, and S. Forchhammer, "Experimental Study of Nonlinear Phase Noise and its Impact on WDM Systems with DP-256QAM," In Proceedings of the $42^{\text {nd }}$ European Conference and Exhibition on Optical Communication, (VDE, 2016), 479-481.

233. X. Tang, Z. Wu, "WDM transmissions exploiting optical phase conjugation," Annales des Télécommunications, 62(5-6), 518-530 (2007).

234. F. Guitierrez, E. Martin, P.Perry, A.D.Ellis, P.Anandarajah, L. Barry, "WDM orthogonal subcarrier multiplexing," Journal of Lightwave Technology, 34(8) 1815-1823 (2016).

235. P.Minzioni, "Nonlinearity compensation in a fiber optic link by optical phase conjugation," Fiber and Integrated Optics, 28(3), 179-209 (2009).

236. K. Solis-Trapala, T. Inoue, and S. Namiki, "Nearly-ideal optical phase conjugation based nonlinear compensation system," in Optical Fiber Communication Conference, OSA Technical Digest Series (Optical Society of America, 2014), paper W3F.8.

237. I.D. Phillips, M. Tan, M. Stephens, M.E. McCarthy, E. Giacoumids, S. Sygletos, P. Rosa, S. Fabbri, S. Le, T. Kanesan, S.K. Turitsyn, N.J. Doran, P. Harper, and A.D. Ellis, "Exceeding the Nonlinear-Shannon Limit using Raman Laser Based Amplification and Optical Phase Conjugation", in Optical Fiber Communication Conference, OSA Technical Digest Series (Optical Society of America, 2014), paper M3C1.

238. J.D. Ania-Castañón, "Quasi-lossless transmission using second-order Raman amplification and fibre Bragg gratings," Opt. Express 12, $4372-4377$ (2004)

239. M. Tan, P. Rosa, S.T. Le, I. D. Phillips, and P. Harper, "Evaluation of 100G DP-QPSK longhaul transmission performance using second order co-pumped Raman laser based amplification," Opt. Express 23, 22181-22189 (2015)

240. M. H. Shoreh, "Compensation of Nonlinearity Impairments in Coherent Optical OFDM Systems Using Multiple Optical Phase Conjugate Modules," J. Opt. Commun. Netw. 6, 549558 (2014)

241. K. Solis-Trapala, M. Pelusi, H. Nguyen Tan, T. Inoue, S. Suda, and S. Namiki, "Approaching Complete Cancellation of Nonlinearity in WDM Transmission Through Optical Phase Conjugation," in Asia Communications and Photonics Conference OSA Technical Digest Series (Optical Society of America, 2015), paper AM3I.2.

242. J. C. Geyer, C. Rasmussen, B. Shah, T. Nielsen, and M. Givehchi, "Power Efficient Coherent Transceivers," In Proceedings of the 42nd European Conference on Optical Communication, (VDE, 2016), 109-111.

243. K. Solis-Trapala, M. Pelusi, H. Nguyen Tan, T. Inoue, and S. Namiki, "Transmission optimized impairment mitigation by 12 stage phase conjugation of WDM $24 \times 48 \mathrm{~Gb} / \mathrm{s} \mathrm{DP}-$ QPSK signals," in Optical Fiber Communication Conference, OSA Technical Digest Series (Optical Society of America, 2015), paper Th3C.2.

244. H. Hu, R. M. Jopson, A. Gnauck, M. Dinu, S. Chandrasekhar, X. Liu, C. Xie, M. Montoliu, S. Randel, and C. McKinstrie, "Fiber Nonlinearity Compensation of an 8-channel WDM PDMQPSK Signal using Multiple Phase Conjugations" in Optical Fiber Communication Conference, OSA Technical Digest Series (Optical Society of America, 2014), paper M3C.2.

245. M.E.McCarthy, M.A.Z. Al Khateeb, and A.D.Ellis, "PMD Tolerant Nonlinear Compensation using In-line Phase Conjugation", Optics Express, 24(4) 3385-3392 (2016).

246. S. L. Jansen, D. van den Borne, B. Spinnler, S. Calabro, H. Suche, P.M. Krummrich, W. Sohler, G.-D. Khoe, and H. de Waardt, "Optical phase conjugation for ultra long-haul phaseshift-keyed transmission," Journal of Lightwave Technology, 24(1), 54-64 (2006).

247. M. D. Pelusi and B. J. Eggleton, "Optically tunable compensation of nonlinear signal distortion in optical fiber by end-span optical phase conjugation," Opt. Express 20, 8015-8023 (2012).

248. M. Morshed, L. B. Du, B. Foo, M. D. Pelusi, B. Corcoran, and A. J. Lowery, "Experimental demonstrations of dual polarization CO-OFDM using mid-span spectral inversion for nonlinearity compensation," Opt. Express 22, 10455-10466 (2014).

249. M. D. Pelusi, "Fiber looped phase conjugation of polarization multiplexed signals for precompensation of fiber nonlinearity effect," Opt. Express 21, 21423-21432 (2013).

250. K. Solis-Trapala, M. Pelusi, H.N. Tan, T. Inoue, and S. Namiki, "Optimized WDM Transmission Impairment Mitigation by Multiple Phase Conjugations," J. Lightwave Technol. 34, 431-440 (2016).

251. D. Vukovic, J. Schröder, F. Da Ros, LB. Du, C.J. Chae, D.-Y. Choi, M. D. Pelusi, and Christophe Peucheret, "Multichannel nonlinear distortion compensation using optical phase conjugation in a silicon nanowire," Opt. Express 23, 3640-3646 (2015). 
252. S. Yoshima, Z. Liu, Y. Sun, K. R. Bottrill, F. Parmigiani, P. Petropoulos, and D. J. Richardson, "Nonlinearity Mitigation for Multi-channel 64-QAM Signals in a Deployed Fiber Link through Optical Phase Conjugation," in Optical Fiber Communication Conference, OSA Technical Digest (Optical Society of America, 2016), paper Th4F.4.

253. S. Namiki, H. Nguyen Tan, K. Solis-Trapala, and T. Inoue, "Signal-transparent wavelength conversion and light-speed back propagation through fiber," in Optical Fiber Communication Conference, OSA Technical Digest (Optical Society of America, 2016), paper Th4F.1.

254. T. Umeki, T. Kazama, A. Sano, K. Shibahara, K. Suzuki, M. Abe, H. Takenouchi, and Y. Miyamoto, "Simultaneous nonlinearity mitigation in $92 \times 180-$ Gbit/s PDM-16QAM transmission over $3840 \mathrm{~km}$ using PPLN-based guard-band-less optical phase conjugation," Opt. Express 24, 16945-16951 (2016).

255. Y. Han, G. Li. "Polarization diversity transmitter and optical nonlinearity mitigation using polarization-time coding", In Coherent Optical Technologies and Applications, OSA Technical Digest Series, (Optical Society of America, 2006), paper CThC7.

256. H. Lu, Y. Mori, C. Han, and K. Kikuchi, "Novel polarization-diversity scheme based on mutual phase conjugation for fiber-nonlinearity mitigation in ultra-long coherent optical transmission systems," In Proceedings of the $39^{\text {th }}$ European Conference and Exhibition on Optical Communication, (IET, 2013), paper We3C3.

257. X. Liu, A.R. Chraplyvy, P. J. Winzer, R. W. Tkach, and S. Chandrasekhar, "Phase-conjugated twin waves for communication beyond the Kerr nonlinearity limit," Nature Photonics 7(7), 560-568 (2013).

258. Y. Tian, Y-K. Huang, S. Zhang, P. R. Prucnal, and T. Wang, "112-Gb/s DP-QPSK transmission over 7,860-km DMF using phase-conjugated copy and digital phase-sensitive boosting with enhanced noise and nonlinearity tolerance," in Optical Fiber Communication Conference, OSA Technical Digest Series (Optical Society of America, 2015), paper Tu2B5.

259. H. Eliasson, P. Johannisson, M. Karlsson, and P. A. Andrekson, "Mitigation of nonlinearities using conjugate data repetition," Opt. Express, 23(3), 2392-2402 (2015).

260. T. Yoshida, T. Sugihara, K. Ishida and T. Mizuochi, "Spectrally-efficient dual phaseconjugate twin waves with orthogonally multiplexed quadrature pulse-shaped signals," in Optical Fiber Communication Conference, OSA Technical Digest Series (Optical Society of America, 2014), paper M3C6.

261. S. L. I. Olsson, B. Corcoran, C. Lundström, M. Sjödin, M. Karlsson and P. A. Andrekson, "Phase-sensitive amplified optical link operating in the nonlinear transmission regime," In Proceedings of the $38^{\text {th }}$ European Conference and Exhibition on Optical Communication, OSA Technical Digest Series (Optical Society of America, 2012), paper Th2F1.

262. B. Corcoran, S. L. I. Olsson, C. Lundström, M. Karlsson and P. A. Andrekson, "Mitigation of nonlinear impairments on QPSK data in phase-sensitive amplified links," In Proceedings of the $39^{\text {th }}$ European Conference and Exhibition on Optical Communication, (IET, 2013), paper We3A1.

263. S.L.I. Olsson B Corcoran, C Lundström, E. Tipsuwannakul, S. Sygletos, A. D. Ellis, Zhi Tong, Magnus Karlsson, and P. A. Andrekson, "Injection-Locking Based Pump Recovery for Phase-Sensitive Amplified Links," Opt. Express., 21(12), 14512-14529 (2013).

264. K. Goroshko, H. Louchet, and A. Richter, "Fundamental Limitations of Digital Back Propagation due to Polarization Mode Dispersion," in Asia Communications and Photonics Conference OSA Technical Digest Series (Optical Society of America, 2015), paper ASu3F.5

265. C. B. Czegledi, G. Liga, D. Lavery, M. Karlsson, E. Agrell, S. J. Savory, and P. Bayvel, "Polarization-Mode Dispersion Aware Digital Backpropagation," in Proceedings of the 42nd European Conference on Optical Communication, (VDE, 2016), 1091-1094 .

266. E. Temprana, E. Myslivets, V. Ataie, B.P.-P. Kuo, N. Alic, S. Radic, V. Vusirikala, and V. Dangui, "Demonstration of Coherent Transmission Reach Tripling by Frequency-Referenced Nonlinearity Pre-compensation in EDFA-only SMF Link," in Proceedings of the 42nd European Conference on Optical Communication, (VDE, 2016), 376-379.

267. T. Healy, F.C. Garcia Gunning, E. Pincemin, B. Cuenot, and A.D. Ellis, "1,200 km SMF (100 km spans) 280 Gbit/s Coherent WDM Transmission using Hybrid Raman/EDFA Amplification", in Proceedings of 33rd European Conference and Exhibition of Optical Communication (VDE, 2007), paper Mo1.3.5.

268. L. B. Du, B. J. Schmidt, and A. J. Lowery, "Efficient Digital Backpropagation for PDM-COOFDM Optical Transmission Systems," in Optical Fiber Communication Conference, OSA Technical Digest Series (Optical Society of America, 2010), paper OTuE2.

269. R. Asif, C. Y. Lin, M. Holtmannspoetter, and B. Schmauss, "Logarithmic step-size based digital backward propagation in N-channel 112Gbit/s/ch DP-QPSK transmission," In 
Proceedings of the 13th International Conference on Transparent Optical Networks, (IEEE, 2011), paper TuP6.

270. M. A. Jarajreh, E. Giacoumidis, I. Aldaya, S. T. Le, A. Tsokanos, Z. Ghassemlooy, and N. J. Doran, "Artificial Neural Network Nonlinear Equalizer for Coherent Optical OFDM," IEEE Photonics Technology Letters, 27(4), 387-390 (2015).

271. P. Poggiolini, G. Bosco, A. Carena, R. Cigliutti, V. Curri, F. Forghieri, R. Pastorelli, and S. Piciaccia, "The LOGON Strategy for Low-Complexity Control Plane Implementation in NewGeneration Flexible Networks," in Optical Fiber Communication Conference, OSA Technical Digest (Optical Society of America, 2013), paper OW1H.3.

272. A. Mecozzi, "On the Optimization of the Gain Distribution of Transmission Lines with Unequal Amplifier Spacing”, Photonics technology Letters, 10(7), 103-106 (1998).

273. D. Rafique and A.D.Ellis, "Various Nonlinearity Mitigation Techniques employing Optical and Electronic Approaches", Photonics Technology Letters, 23(23), 1041-1044, (2011).

274. I. Kim, O. Vassilieva, P. Palacharla, and M. Sekiya, "The impact of spectral inversion placement for nonlinear phase noise mitigation in non-uniform transmission links," In Proceedings of 2014 IEEE Photonics Conference, (IEEE, 2014), 146-147.

275. R.G. Smith, "Optical power handling capacity of low loss optical fibers as determined by stimulated Raman and Brillouin scattering," Applied Optics 11(11), 2489-2494 (1972).

276. D. Cotter, "Observation of stimulated Brillouin scattering in low-loss silica fibre at $1.3 \mu \mathrm{m}$," in Electronics Letters, 18(12), 495-496 (1982).

277. T. Sugie, "Maximum repeaterless transmission of lightwave systems imposed by stimulated Brillouin scattering in fibres," Optical and Quantum Electronics, 27(7), 643-661 (1995).

278. A. R. Chraplyvy, "Limitations on lightwave communications imposed by optical-fiber nonlinearities," Journal of Lightwave Technology, 8(10), 1548-1557 (1990).

279. D. Cotter, "Optical Transmission," Patent EP0099632A1, 1984.

280. E.G.Bryant, A.D.Ellis, W.A.Stallard, S.F.Carter, J.V.Wright, and R.Wyatt "Unrepeatered transmission over $250 \mathrm{~km}$ of step index fibre using erbium power amplifier," Electronics Letters, 26(8), 528-529 (1990).

281. Y. Aoki, K. Tajima, and I. Mito, "Input power limits of single-mode optical fibers due to stimulated Brillouin scattering in optical communication systems," in Journal of Lightwave Technology, 6(5), 710-719 (1988).

282. T. Sugie, "Impact of SBS on CPFSK coherent transmission systems using dispersion-shifted fiber," IEEE Photonics Technology Letters, 5(1), 102-105 (1993).

283. K. Yonenaga, S. Kuwano, S. Norimatsu, and N. Shibata, "Optical duobinary transmission system with no receiver sensitivity degradation," Electronics Letters, 31(4), 302-304 (1995).

284. L. Galdino, D. Semrau, D. Lavery, G. Saavedra, C. B. Czegledi, E. Agrell, R. I. Killey, and P. Bayvel, "On the limits of digital back-propagation in the presence of transceiver noise," Opt. Express 25, 4564-4578 (2017).

285. R. Hui, C. Laperle, A. D. Shiner, M. Reimer, and M. O'Sullivan, "Characterization of electrostriction nonlinearity in a standard single-mode fiber based on cross-phase modulation," in Optical Fiber Communication Conference, OSA Technical Digest Series (Optical Society of America, 2015), paper W2A.38.

286. J. Bromage, "Raman amplification for fiber communications systems," Journal of Lightwave Technology 22(1), 79-93 (2004).

287. D.R. Solli, C. Ropers, P. Koonath, and B. Jalali, “Optical rogue waves,” Nature, 450(7172), 1054-1057 (2007).

288. S. Vergeles, and S. K. Turitsyn. "Optical rogue waves in telecommunication data streams," Physical Review A 83(6), 061801 (2011).

289. X. Zhou and M. Birk, "Performance limitation due to statistical Raman crosstalk in a WDM system with multiple-wavelength bidirectionally pumped Raman amplification," Journal of lightwave technology 21(10) 2194 (2003).

290. A.R. Chraplyvy, "Optical power limits in multi-channel wavelength-division-multiplexed systems due to stimulated Raman scattering," Electronics letters 20(2), 58-59 (1984).

291. K. Rottwitt, J. Bromage, J.,A.J. Stentz, L. Leng, M.E. Lines, and H. Smith, "Scaling the Raman gain coefficient: Applications to Germanosilicate fibers," Journal of Lightwave Technology, 21(7), 1652-1663 (2003).

292. X.Liu and Y. Li, "Optimizing the bandwidth and noise performance of distributed multi-pump Raman amplifiers," Optics communications 230(4), 425-431 (2004).

293. D.N. Christodoulides and R. B. Jander. "Evolution of stimulated Raman crosstalk in wavelength division multiplexed systems," IEEE Photonics Technology Letters 8(12) 17221724 (1996). 
294. K.-P. Ho, "Statistical properties of stimulated Raman crosstalk in WDM systems." Journal of Lightwave Technology 18(7), 915-921, (2000).

295. D. Cotter and A. M. Hill. "Stimulated Raman crosstalk in optical transmission: Effects of group velocity dispersion," Electronics Letters 20(4), 185-187 (1984).

296. N.R. Das and S. Sarkar, "Probability of power depletion in SRS cross-talk and optimum detection threshold for minimum BER in a WDM receiver," IEEE Journal of Quantum Electronics 47(4), 424-430 (2011).

297. G. Saavedra, M. Tan, D. J. Elson, L. Galdino, D. Semrau, M. A. Iqbal, I. Phillips, P. Harper, N. MacSuibhne, A. Ellis, D. Lavery, B. C. Thomsen, R. Killey, and P. Bayvel, "Experimental Investigation of Nonlinear Signal Distortions in Ultra-Wideband Transmission Systems," in Optical Fiber Communication Conference, OSA Technical Digest Series (Optical Society of America, 2017), paper W1G.1

298. A.D.Ellis and S. Sygletos, "The Potential for Networks with Capacities Exceeding the Nonlinear Shannon Limit", in Photonic Networks and Devices (PND), OSA Technical Digest Series (Optical Society of America, 2015), paper NeT2F1

299. On-line source: http://reference.wolfram.com/language/note/CountryDataSourceInformation.html, accessed $18^{\text {th }}$ March 2017.

300. S.K.Routray, R.M.Morais, J.R.Ferreira da Rocha, A.N.Pinto, "Statistical Model for Link Lengths in Optical Transport Networks”, J.Opt.Comm.Netw., 5(7), 762-773 (2013).

301. D. J. Ives, A. Alvarado, and S. J. Savory, "Throughput Gains From Adaptive Transceivers in Nonlinear Elastic Optical Networks," Journal of Lightwave Technology, 35(6), 1280-1289 (2017).

302. C. Sanchez, M. Mccarthy, A. D. Ellis, P. Wright, and A. Lord, "Optical-phase conjugation nonlinearity compensation in Flexi-Grid optical networks," In Proceedings of Recent Advances on Systems, Signals, Control, Communications and Computers, (WSEAS, 2015), $39-43 /$

303. D.J.Richardson, J. M. Fini, and L. E. Nelson. "Space-division multiplexing in optical fibres." Nature Photonics 7(5), 354-362 (2013).

304. M. Mazurczyk, J.X. Cai, H.G. Batshon, Y. Sun, O.V. Sinkin, M.A. Bolshtyansky, and A. Pilipetskii, "50GBd 64APSK Coded Modulation Transmission Over Long Haul Submarine Distance with Nonlinearity Compensation and Subcarrier Multiplexing," in Optical Fiber Communication Conference, OSA Technical Digest Series (Optical Society of America, 2017), paper Th4D5.

305. Y. Sun, "SDM for Power Efficient Transmission," in Optical Fiber Communication Conference, OSA Technical Digest Series (Optical Society of America, 2011), paper M2F1.

306. G. Kramer, M.I. Yousefi, and F.R Kschischang. "Upper bound on the capacity of a cascade of nonlinear and noisy channels," In Proceedings of the 2015 IEEE Information Theory Workshop (ITW), (IEEE, 2015), paper 7133167.

307. R. M. Percival, E.S.R. Sikora, and R. Wyatt, "Catastrophic damage and accelerated ageing in bent fibres caused by high optical powers." Electronics Letters 36(5), 414-416 (2000).

308. N. M. Suibhne, F. M. Ferreira, M. E. McCarthy, A. Mishra, and A. D. Ellis, "The effect of high optical power on modern fibre at $1.5 \mu \mathrm{m}, "$, " In Proceedings of the 18th International Conference on Transparent Optical Networks, (IEEE, 2016), paper TuP24.

309. D. Lavery, R. Maher, D. Millar, A. Alvarado, S. J. Savory, and P. Bayvel "Why compensating fibre nonlinearity will never meet capacity demands," arXiv preprint arXiv:1512.03426 (2015). 


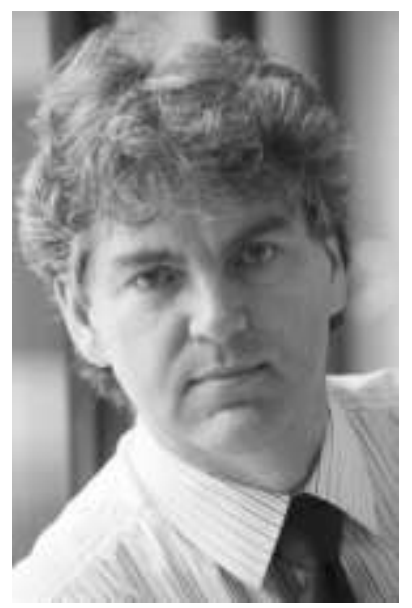

Andrew D. Ellis was born in Underwood, U.K., in 1965. He received the B.Sc. degree in physics with a minor in mathematics from the University of Sussex, Brighton, U.K., in 1987. He received the Ph.D. degree in electronic and electrical engineering from The University of Aston in Birmingham, Birmingham, U.K., in 1997 for his study on all optical networking beyond $10 \mathrm{Gbit} / \mathrm{s}$.

He previously worked for British Telecom Research Laboratories as a Senior Research Engineer investigating the use of optical amplifiers and advanced modulation formats in optical networks and the Corning Research Centre as a Senior Research Fellow where he led activities in optical component characterization. From 2003, he headed the Transmission and Sensors Group at the Tyndall National Institute in Cork, Ireland, where he was also a member of the Department of Physics, University College Cork and his research interests included the evolution of core and metro networks, and the application of photonics to sensing. He is now 50th Anniversary Professor of Optical Communications at Aston University where he is also deputy director of the Institute of Photonics Technologies (AiPT), and he holds adjunct professorships from University College Cork (Physics) and Dublin City University (RINCE). He has published over 200 journal papers and over 28 patents in the field of photonics, primarily targeted at increasing capacity, reach and functionality in the optical layer.

Prof. Ellis is a member of the Institute of Physics and a Chartered Physicist. He served for 6 years as an associate editor of the journal Optics Express. Prof Ellis has been a member of the Technical Program Committee of ECOC since 2004 and two three year terms on the TPC of OFC. He is currently participating in the organization of ECOC 2019.

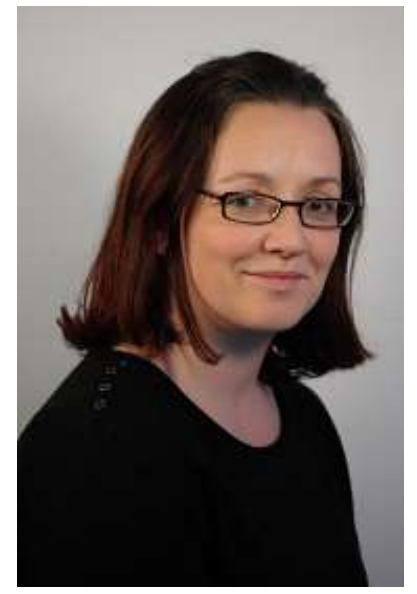

Mary E. McCarthy was born in Cork, Ireland. She received the B.Eng. degree in electrical and electronic engineering from University College Cork, Cork, Ireland, in 2004. She received her PhD degree in Laser and Optical Engineering in 2009 from the Department of Physics, University College Cork for her thesis entitled "Phase estimation receiver for full-field detection", where she was also affiliated with the Photonics Systems Group at the Tyndall National Institute.

She previously worked Ericsson in both the UK and Australia on the application of wavelength division multiplexing to commercial communication systems, participating across a wide range of applications from product development to installation training. In 2013 she joined the Aston institute of Photonic Technologies at Aston University where her research interests included digital signal processing applied to optical communication systems, and optical phase conjugation for the mitigation of nonlinear transmission effects. She is now with Oclaro, Paignton. Dr McCarthy is a member of the Institute of Engineering Technology, and has published over 60 papers in leading engineering journals and conferences. 


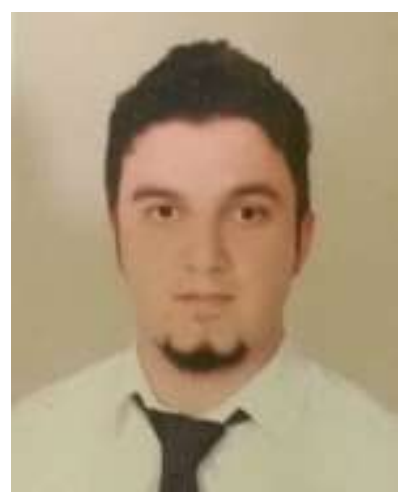

Mohammad Ahmad Zaki Al-Khateeb was born in Irbid, Jordan, in 1989. He received the B.S. in Communication and Software Engineering from Balqa' Applied University, Jordan. Then he received M.S. degrees in Photonics Networks Engineering (MAPNET), Erasmus Mundus double master degree, from Scuola Superiore Sant'Anna and Aston University. Mohammad is currently working towards his PhD degree from Aston University under the supervision of Prof. Andrew Ellis, researching the ability to expand the capacity of optical fiber transmission systems through nonlinearity compensation techniques. He has authored/co-authored over 12 journal and conference papers on electrical and all-optical nonlinearity compensation techniques in optical transmission systems.

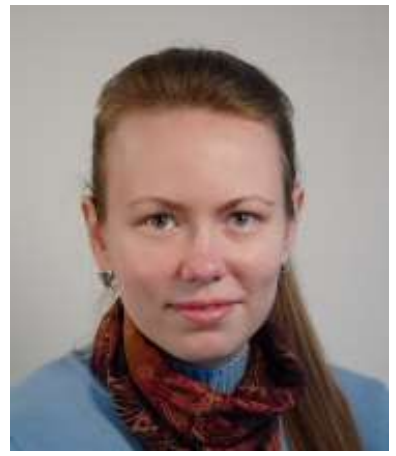

Mariia Sorokina is a Research Fellow at the Aston Institute of Photonic Technologies. She received the M.Sc. degree in theoretical physics from V. N. Karazin Kharkiv National University, Kharkiv, Ukraine in 2010, which resulted in two publications on condensed matter physics and nonlinear optical effects in metamaterials. She then moved to the optical communication and information theory and received her $\mathrm{PhD}$ degree in Mathematics in 2014 from the Aston Institute of Photonics Technologies, Aston University. Since then she is working as Aston University where her main areas of research include information theory, fibre-optic communication, and all-optical regeneration. Dr. Sorokina has published over 30 papers in leading journals and conferences, made over 15 invited talks (including prestigious CLEO conference), and has three patents.

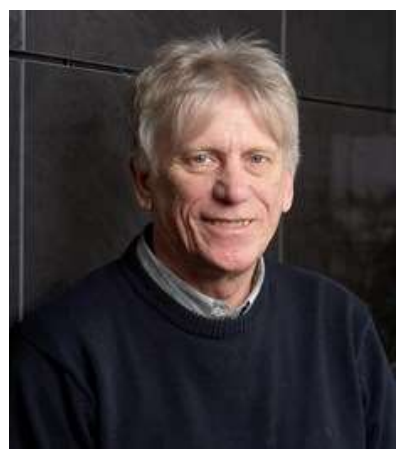

Nick J. Doran has over 35-years research experience in high-speed and long distance optical communications. He led a research team at BT for 10 years on both theoretical and experimental investigations in ultra-high speed optical systems from 1981 to 1991. He jointly established the photonics research group at Aston University 19912000 specializing in soliton communication and processing. During this time the research was extensively funded by EPSRC and supported by industrial contracts with Marconi and KDD. In 2000 he established a start-up development within Marconi (SOLSTIS) to develop an ultralong communication system based on his research on dispersion managed solitons. In 2005 he took on the role of Head and Director of the Institute of Advanced Telecommunication (IAT) at Swansea University. He returned to Aston University in November 2013 and now runs key research projects on nonlinear fibre amplification and optical networks. Prof Doran has published over 200 papers and 20 patents on optical transmission and processing. He invented the concept of dispersion managed solitons and the extensively used Nonlinear Optical Loop Mirror (NOLM). 